\title{
1 TopBP1 assembles nuclear condensates to switch on ATR signalling.
}

2 Camilla FRATTINI ${ }^{1}$, Alexy PROMONET ${ }^{1}$, Emile ALGHOUL ${ }^{1}$, Sophie VIDAL-EYCHENIE ${ }^{1}$,

3 Marie LAMARQUE ${ }^{1}$, Marie-Pierre BLANCHARD ${ }^{1}$, Serge URBACH ${ }^{2}$, Jihane BASBOUS ${ }^{1,3}$

4 and Angelos CONSTANTINOU ${ }^{1,3,4}$

5

91 Institut de Génétique Humaine - CNRS-Université de Montpellier

102 Institut de Génomique Fonctionnelle - CNRS INSERM - Université de Montpellier

11

12

133 Correspondance : jihane.basbous@igh.cnrs.fr; angelos.constantinou@igh.cnrs.fr

144 Lead contact

15 


\section{Abstract}

18 ATR checkpoint signalling is crucial for cellular responses to DNA replication

19 impediments. Using an optogenetic platform, we show that TopBP1, the main activator of

20 ATR, self-assembles extensively to yield micron-sized condensates. These opto-TopBP1

21 condensates are functional entities organized in tightly packed clusters of spherical nano-

22 particles. TopBP1 condensates are reversible, occasionally fuse and co-localise with

23 TopBP1 partner proteins. We provide evidence that TopBP1 condensation is a molecular

24 switch that amplifies ATR activity to phosphorylate checkpoint kinase 1 (Chk1) and

25 slowdown replication forks. Single amino acid substitutions of key residues in the

26 intrinsically disordered ATR-activation domain disrupt TopBP1 condensation and,

27 consequently, ATR/Chk1 signalling. In physiologic salt concentration and pH, purified

28 TopBP1 undergoes liquid-liquid phase separation in vitro. We propose that the actuation

29 mechanism of ATR signalling is the assembly of TopBP1 condensates driven by highly

30 regulated multivalent and cooperative interactions. 


\section{Introduction}

32 The primary structure of DNA is subjected to constant chemical alterations caused by

33 spontaneous decay, endogenous metabolites and environmental genotoxic agents (Friedberg et

34 al., 2006; Lindahl, 1993), hence organisms have evolved multiple DNA repair mechanisms to

35 ensure genome integrity and survival (Ciccia and Elledge, 2010; Jackson and Bartek, 2009;

36 Tubbs and Nussenzweig, 2017). DNA damage sensors, protein scaffolds and DNA processing

37 activities accumulate at DNA damage sites to form spatially defined and reversible structures not

38 delimited by membranes commonly referred to as nuclear foci (Garcia-Higuera et al., 2001;

39 Lisby et al., 2001; Maser et al., 1997; Park et al., 1996). Much still remains to be understood

40 about the molecular forces that drive the formation of DNA damage foci and their functional

41 consequences in the DNA damage response.

In recent years, application of the principles of polymer chemistry to biological

43 molecules has accelerated spectacularly our understanding of how membraneless compartments

44 assemble and regulate their compositions and functions (Banani et al., 2017; Bracha et al., 2019;

45 Hyman and Simons, 2012; Shin and Brangwynne, 2017; Soding et al., 2020). These self-

46 organized micron-scale structures, called biomolecular condensates, assemble via multiple weak,

47 cooperative and dynamic interactions and compartmentalize proteins and nucleic acids. The self-

48 organization of multivalent molecules can yield a rich repertoire of higher-order structures with

49 diverse physical properties, variable size, and no defined stoichiometry of constituent proteins.

50 Whereas nucleic acids serve a seeding platform for the self-organization of soluble proteins

51 (Mao et al., 2011; McSwiggen et al., 2019), soluble bridging factors can cross-link chromatin

52 segments to compartmentalize chromatin via a process of polymer-polymer phase separation

53 (Erdel et al., 2020). Moreover, increasing evidence indicates that diverse multivalent protein 
54 scaffolds self-organize via liquid-liquid phase separation, a process of de-mixing that yields a

55 condensed phase enriched in the protein and a dilute phase (Banani et al., 2017; Shin and

56 Brangwynne, 2017). Biomolecular condensates are functional hubs implicated in diverse cellular

57 processes, including innate immune signalling ( $\mathrm{Du}$ and Chen, 2018), microtubule nucleation

58 (Woodruff et al., 2017), transcription (Boija et al., 2018; Kwon et al., 2013; Lu et al., 2018;

59 Sabari et al., 2018) and adaptative stress responses (Franzmann and Alberti, 2019; Franzmann et

60 al., 2018; Riback et al., 2017). Recent evidence indicates that protein phase separation can also

61 occur at DNA damage sites. Upon laser micro-irradiation, the activity of poly(ADP-ribose)

62 polymerase 1 seeds the condensation of the prototypical liquid-liquid phase separation protein

63 FUS at damaged chromatin (Altmeyer et al., 2015; Patel et al., 2015). The DNA repair protein

64 53BP1 phase separates at double-strand DNA breaks (Kilic et al., 2019; Pessina et al., 2019), and

65 the condensation of 53BP1 promotes induction of p53 and p21 (Kilic et al., 2019).

To explore the mechanisms and functional consequences of biomolecular condensates in

67 the DNA damage response (DDR), we studied Topoisomerase II $\beta$-binding protein (TopBP1), an

68 essential factor in the DDR pathway and a prototype of protein scaffolds composed of multiple

69 modular interaction domains. TopBP1 features nine repetitions of a well-folded protein-protein

70 interaction motif, the BRCA1 $\mathrm{C}$ terminus domain (BRCT), and an ATR activation domain

71 (AAD), located between BRCT6 and BRCT7, which is intrinsically disordered. In a highly

72 regulated manner, TopBP1 brings together different sets of proteins to form distinct protein

73 complexes involved in DNA replication initiation (Hashimoto and Takisawa, 2003; Makiniemi et

74 al., 2001), DNA replication stress signalling (Kumagai et al., 2006; Mordes et al., 2008), DNA

75 repair (Broderick et al., 2015; Leimbacher et al., 2019; Liu et al., 2017; Moudry et al., 2016) and

76 transcription regulation (Liu et al., 2009; Wright et al., 2006). In S phase, TopBP1 is the main 
77 activator of the master checkpoint kinase ATR (Kumagai et al., 2006; Mordes et al., 2008). ATR

78 and its effector kinase Chk1 are crucial for cellular responses to DNA damage and DNA

79 replication impediments (Ciccia and Elledge, 2010; Marechal and Zou, 2013; Saldivar et al.,

80 2017). ATR/Chk1 signalling ensures cell and organismal survival through coordination of DNA

81 repair and DNA replication with physiological processes, including cell cycle progression and

82 transcription (Saldivar et al., 2017). Studies using Xenopus egg extracts have largely contributed

83 to defining the orchestrated set of events leading to ATR activation (Acevedo et al., 2016; Byun

84 et al., 2005; Duursma et al., 2013; Kumagai et al., 2006; Van et al., 2010). ATR is recruited to

85 DNA lesions and replication intermediates via the ATR interacting protein ATRIP, which binds

86 RPA-covered single-stranded DNA (Zou and Elledge, 2003). TopBP1 interacts with ATRIP,

87 with ATR and with the RAD9-RAD1-HUS1 (9-1-1) clamp and activates ATR (Delacroix et al.,

88 2007; Duursma et al., 2013; Kumagai et al., 2006; Mordes et al., 2008; Yan and Michael, 2009).

89 Here, we used the conceptual framework born from studies of biomolecular condensates to gain

90 fresh insights into the activation mechanism of the ATR/Chk1 signalling pathway. Using a

91 combination of optogenetic and biochemical approaches, we demonstrate that TopBP1 can self-

92 assemble extensively to yield functional biomolecular condensates. We show that purified

93 TopBP1 has an intrinsic capacity to undergo liquid phase separation in vitro. Moreover, we

94 provide evidence that TopBP1 self-organization into micron-sized compartments in living cells

95 activates ATR/Chk1 signalling and slows down the progression of replication forks. Our data

96 indicate that essential responses to DNA replication impediments emerge from TopBP1-driven

97 assembly of nuclear condensates. 


\section{Results}

99 TopBP1 drives the formation of micron-sized nuclear condensates.

100 To probe the capacity of TopBP1 to self-organize into biomolecular condensates, we fused

101 TopBP1 to cryptochrome 2 (Cry2) of Arabidopsis thaliana, a protein that oligomerises upon

102 exposure to 488nm light (Bugaj et al., 2013) (Fig. 1A). This optogenetic system allows to control

103 the nucleation of biomolecular condensates in space and time (Bracha et al., 2018; Shin et al.,

104 2017; Shin et al., 2018), and evaluate the functional consequences of protein condensation (Kilic

105 et al., 2019; Sabari et al., 2018). When fused to a protein scaffold that can undergo phase

106 separation, the light-responsive Cry2 protein seeds the formation of biomolecular condensates

107 that are held together by multivalent, weak and cooperative interactions (Kilic et al., 2019; Shin

108 et al., 2017; Zhang et al., 2019). We induced the expression of TopBP1 fused to mCherry and

109 Cry2 (named opto-TopBP1) with doxycycline in Flp-In HEK293 cells. Overall, the expression

110 level of recombinant opto-TopBP1 was similar to the level of endogenous TopBP1 (Suppl.

111 Figure 1A). At this level of expression, opto-TopBP1 remained in a diffuse state and was not

112 directly detectable by fluorescence microscopy (Figure 1B, light OFF panel). Upon exposure of

113 these cells to an array of blue-light LEDs during 3 minutes of light-dark cycles (4s light followed

114 by 10 s dark), we observed multiple and distinct opto-TopBP1 foci in the nuclei, specifically

115 (Figure 1B, light ON panel). By contrast, we did not detect any foci in blue-light exposed cells

116 expressing the opto-module (mCherry-Cry2) alone, nor in cells expressing the checkpoint clamp

117 subunit opto-RAD9 (RAD9-mCherry-Cry2) (Suppl. Figure 1B). We compared cells expressing

118 WT or W1145R opto-TopBP1. This mutation locates inside TopBP1 ATR activation domain

119 (AAD), an intrinsically disordered domain required to directly stimulate the kinase activity of

120 ATR (Kumagai et al., 2006). The tryptophan to arginine substitution W1138R in the ATR 
121 activation domain of Xenopus Laevis TopBP1 suppresses its capacity to activate Xenopus ATR

122 (Kumagai et al., 2006). Furthermore, the homologous substitution W1147R is embryonic lethal

123 in mice (Zhou et al., 2013). Upon optogenetic activation, W1145R TopBP1 expressing cells

124 exhibited a markedly reduced number of TopBP1 condensates in comparison with wild-type

125 TopBP1 (Figure 1B), suggesting that this aromatic residue plays an important role in TopBP1

126 higher-order assembly or the growth of TopBP1 condensates.

127 Besides TopBP1, Ewing's tumor associated antigen 1 (ETAA1) has been recently

128 identified as necessary to maintain Chk1 basal activity and stability (Michelena et al., 2019), and

129 for the regulation of mitotic ATR signalling (Bass and Cortez, 2019). Like TopBP1, ETAA1 is

130 endowed with an intrinsically disordered ATR activation domain (AAD) (Bass and Cortez, 2019;

131 Bass et al., 2016; Haahr et al., 2016; Lee et al., 2016). Considering the conserved function and

132 disordered nature of these ATR activation domains, we replaced the AAD of TopBP1 with the

133 AAD of ETAA1, and then tested the capacity of the chimeric opto-TopBP1 ${ }^{\text {ETAA1-AAD }}$ protein to

134 form condensates. Exposure of these chimeric proteins to blue light did not yield condensates

135 (Figure 1C). Consistent with this observation, ETAA1 did not form foci when fused to Cry2 and

136 exposed to blue light (Suppl. Figure 1C). Furthermore, in isolation, the AAD of TopBP1 fused to

137 Cry2 did not yield condensates upon optogenetic activation (Figure 1D). Collectively, these data

138 indicate that TopBP1 can self-assemble into micron-sized condensates and that TopBP1 AAD

139 has unique characteristic features that are necessary but not sufficient for TopBP1 higher-order

140 assembly. 


\section{Optogenetic and endogenous TopBP1 condensates share similar properties.}

145 To understand the nature of TopBP1 foci, we used the conceptual framework of biomolecular

146 condensates to explore their properties. A three-dimensional analysis revealed that

147 optogenetically-induced TopBP1 condensates have a spherical shape with an aspect ratio close to

148 one, as if the interface of these structures was subjected to surface tension (Figure $2 \mathrm{~A}+\mathrm{Suppl}$.

149 video). Furthermore, opto-TopBP1 condensates occasionally fused, suggesting that TopBP1

150 undergoes dynamic clustering (Figure 2B). As expected for membraneless compartments that are

151 held together by multiple weak and transient interactions, opto-TopBP1 foci were reversible

152 (Figure 2C + Suppl. Figure 2A), yet dissolved within 15 minutes, while Cry2 oligomers

153 disassemble spontaneously within 1-2 minutes (Shin et al., 2017). The relative stability of opto-

154 TopBP1 condensates reflects the role of TopBP1 multivalent cooperative interactions in the

155 formation of micron-sized protein assemblies. Next, we assessed the permeability of TopBP1

156 condensates to the surrounding milieu by fluorescence recovery after photobleaching, using an

157 experimental system developed by Sokka et al., 2015. We induced expression of eGFP-TopBP1

158 in U-2-OS cells with doxycycline for 24 hours. In these experimental conditions, overexpressed

159 eGFP-TopBP1 spontaneously forms foci that accumulate in nucleoli (Sokka et al., 2015). After

160 photobleaching, eGFP-TopBP1 bodies recovered fluorescence signal within seconds, which

161 reflects the rapid exchange of eGFP-TopBP1 molecules between the nucleoplasm and TopBP1

162 nuclear condensates (Suppl. Figure 2B). We conclude that the boundaries of TopBP1

163 compartments are permeable. We also noted that eGFP-TopBP1 condensates were sensitive to

164 hexanediol, an aliphatic alcohol that disrupts weak hydrophobic interactions (Suppl. Figure 2C).

165 The cooperative interactions that drive the formation of biomolecular condensates in

166 physiological conditions are determined by protein sequences and by the properties of the 
167 surrounding milieu. Changes in osmotic concentration by addition of sorbitol or sucrose in the

168 cell culture medium disrupt 53BP1 phase separation (Kilic, 2019). Likewise, osmotic stress

169 inhibited both the assembly of TopBP1 condensates induced by optogenetic activation (Figure

$1702 \mathrm{D}+$ Suppl. Figure 2D), and the formation of endogenous TopBP1 foci induced by cellular

171 treatment with the inhibitor of ribonucleotide reductase hydroxyurea (Figure $2 \mathrm{E}+\mathrm{Suppl}$. Figure

172 2E). This suggests that electrostatic forces drive the assembly of both synthetic (optogenetic) and

173 endogenous TopBP1 condensates. Furthermore, recombinant opto-TopBP1 also formed nuclear

174 foci when cells were exposed to hydroxyurea, rather than 488nm light (Suppl. Figure 2F),

175 indicating that like endogenous TopBP1, opto-TopBP1 is mobilized and engages with

176 endogenous components in response to stalled replication forks. TopBP1 condensates appear as

177 homogenous structures by conventional fluorescence microscopy. We used stimulated emission

178 depletion (STED) nanoscopy to gain better insights into the sub-structural organization of

179 TopBP1 condensates. High-resolution imaging using an anti-mCherry antibody revealed the

180 underlying sub-structure of optogenetic TopBP1 condensates, which consist in clusters of

181 spherical, nanometer-sized particles (Figure 2F, left panel). TopBP1 clusters had a very tight

182 appearance when detected with an anti-TopBP1 antibody that recognizes both endogenous and

183 recombinant TopBP1 (Figure 2F, right panel). High-resolution imaging of endogenous TopBP1

184 foci induced by cellular treatment with hydroxyurea identified similar sub-structured clusters of

185 nano-condensates (Figure 2G). Collectively, the data suggest that whether seeded by DNA

186 replication impediments or Cry2 oligomerisation, the driving forces and the organization of

187 TopBP1 nuclear condensates are similar. 


\section{TopBP1 undergoes liquid-liquid phase separation in vitro.}

191 Multivalent protein scaffolds that drive the formation of biomolecular condensates often phase

192 separate in vitro. To test if TopBP1 has the capacity to phase separate in physiologic salt and $\mathrm{pH}$,

193 we expressed the carboxy-terminal half of TopBP1 (amino acids 884-1522) in insect cells. This

194 portion of TopBP1, hereafter referred to as b6-8, includes the BRCT 6 to 8 and the intrinsically

195 disordered ATR activation domain (AAD), located between BRCT6 and BRCT7 (Figure 3A).

196 TopBP1 $1^{\mathrm{b} 6-8}$ mediates directly the activation of ATR, independently of its amino-terminal portion,

197 which is required for the initiation of DNA replication (Hashimoto et al., 2006; Kumagai et al.,

198 2006). TopBP $1^{\mathrm{b} 6-8}$ formed optogenetically-induced foci in live cells (Suppl. Figure 3A). In the

199 presence of the crowding agent Polyethylene Glycol (2\%), purified TopBP $1^{\mathrm{b}-8}$-GFP readily

200 formed spherical condensates detected by fluorescence microscopy (Figure 3B, upper panel and

201 Suppl. Figure 3B). Consistent with optogenetic experiments (Figure 1B), the W1145R

202 substitution abolished the phase separation of TopBP1 ${ }^{\text {b6-8 }}$ (Figure 3B, lower panel). Addition of

203 TopBP1 $1^{\text {b6-8 }}$-RFP to pre-formed TopBP1 ${ }^{\text {b6-8 }}$-GFP condensates yielded yellow compartments,

204 suggesting that soluble TopBP1 molecules are recruited to TopBP1 condensates. By contrast,

205 W1145R TopBP1 ${ }^{\text {b6-8 }}$-RFP did not stably associate with TopBP1 ${ }^{\text {b6-8 }}$-GFP condensates (Figure

206 3C). TopBP1 condensates were permeable to DNA, as revealed by the partitioning of double-

207 strand DNA fragments into pre-formed TopBP1 condensates (Figure 3D). In the presence of a

208 circular DNA plasmid (2.9 kb), TopBP $1^{\mathrm{b} 6-8}$-GFP formed clusters of nano-condensates that were

209 reminiscent, yet not equivalent, to high-resolution images of cellular TopBP1 condensates

210 (Figure 3E). This observation suggests that the long anionic DNA polymer strongly influences

211 the assembly of TopBP1 molecules. In isolation and in the absence of PEG, purified TopBP1 ${ }^{\text {b6-8 }}$ -

212 GFP $(10 \mu \mathrm{M})$ did not phase separate (Figure 3B minus PEG, and 3F). To recapitulate partially the 
213 complex environment of the nucleus, we spiked "Dignam and Roeder" nuclear extracts with

214 recombinant TopBP1 ${ }^{\mathrm{b} 6-8}$-GFP in physiologic salt concentration and $\mathrm{pH}$. After incubation of the

215 reaction mixture at $37^{\circ} \mathrm{C}$, fluorescence microscopy analyses revealed $\mu \mathrm{m}$-scaled TopBP ${ }^{\mathrm{b} 6-8}-\mathrm{GFP}$

216 condensates (Figure 3F) co-localising with endogenous DNA (Suppl. Figure 3C). Altogether, the

217 data suggest that TopBP1 foci-like structures are recapitulated with purified TopBP1 in the

218 presence of a crowding agent or nuclear extracts. Consistent with optogenetic experiments in

219 living cells, TopBP $1^{\mathrm{b} 6-8}$-GFP condensation in nuclear extracts depends on the integrity of the

220 AAD. We thus conclude that TopBP1, both in vitro and in living cells, has intrinsic capacity to

221 form micron-sized condensates.

223 Consequence of TopBP1 condensation on TopBP1-associated protein network.

224 To test if recombinant TopBP1 condensates mimic endogenous TopBP1 foci, we analyzed the

225 composition of TopBP1 condensates assembled in live cells. We used a biotin labelling approach

226 with a low temporal resolution to gain a panoramic view of TopBP1 proximal proteins. A

227 doxycycline-inducible cDNA encoding wild-type or W1145R TopBP1 fused to the mutated

228 biotin ligase BirA* and the Flag epitope was stably integrated in Flp-In HEK293 cells (Figure

229 4A). We overexpressed Flag-BirA*-TopBP1 with doxycycline for 16 hours to a level that

230 induces constitutive BirA*-TopBP1 condensates (Suppl. Figure 4), in the absence of DNA

231 damaging agents. We labelled TopBP1 proximal proteins with biotin for 3 hours. More than 500

232 TopBP1 proximal proteins were identified by mass spectrometry with high confidence and

233 reproducibility (Supplemental excel sheet). Proteins were ranked according to their iBAQ value

234 (intensity-based absolute quantification), a proxy for protein abundance (Figure 4B). Among

235 abundant TopBP1 proximal proteins, we identified known TopBP1 partners, including TOP2A, 
236 FANCJ, BRCA1, NBS1, MRE11, MDC1, 53BP1 and BLM (Figure 4B). Only five proteins

237 showed differences in abundance between wild-type and W1145R TopBP1 (Log2 diff $>2$ with a

238 p-value $\leq$ 0.05) (Figure 4C). Among them, only the nucleolar protein NOL11 was detected at

239 high level, and, therefore, considered as a significant difference between wild-type and W1145R

240 TopBP1. This observation is consistent with a previous report demonstrating that upregulated

241 TopBP1 accumulates on ribosomal chromatin, segregates nucleolar components and yields

242 nucleolar caps (Sokka et al., 2015). By contrast, W1145R substitution abrogates TopBP1

243 nucleolar segregation (Sokka et al., 2015). Consistent with this, W1145R TopBP1 had lost

244 proximity with the nucleolar protein NOL11 (Figure 4D). Immunoblot analysis of TopBP1

245 proximal proteins confirmed that the substitution W1145R in the AAD of TopBP1 does not alter

246 the proximity of TopBP1 with partner proteins implicated in ATR signalling, including ATR,

247 BRCA1, MRE11 and FANCJ (Figure 4D). In sum, the substitution W1145R does not alter

248 significantly the network of TopBP1 proximal proteins. In conclusion, at a low temporal

249 resolution, whereas the composition of recombinant TopBP1 assemblies recapitulates

250 endogenous TopBP1 foci, TopBP1 condensation does not increase significantly the association

251 of TopBP1 with partner proteins.

253 Functional consequences of TopBP1 condensation.

254 The optogenetic switch described above allows controlling TopBP1 assembly by light, which

255 bypasses the confounding effects of prolonged cellular treatments with DNA damaging agents.

256 In response to DNA replication impediments, ATR activates the effector checkpoint kinase 1

257 (Chk1) by phosphorylation on Ser345. To establish a functional link between TopBP1

258 condensation and ATR/Chk1 signalling, we exposed a cell culture dish to an array of blue-light 
LEDs for 3 minutes and then probed cell extracts for ATR mediated Chk1 phosphorylation on

260 Ser345 by western blotting. Optogenetic condensation of TopBP1 induced robust

261 phosphorylation of Chk1 Ser345 (Figure 5A) and of TopBP1 on Ser1138 (Figure 5B, t3), within

2623 minutes, in the absence of an exogenous source of DNA damage. By contrast, the clustering

263 defective W1145R TopBP1 mutant protein did not activate ATR/Chk1 signalling (Figure 5A).

264 Consistent with the progressive dissolution of optogenetically-induced TopBP1 foci, phospho-

265 Chk1 (Ser345) immunoblotting signals disappeared twenty minutes after optogenetic activation

266 (Figure 5B, t23). Re-activation of TopBP1 condensates with blue light re-induced Chk1

267 phosphorylation (Figure 5B, t26), until dissolution of the reactivated TopBP1 condensates

268 (Figure 5B, t46). We reiterated the process four times (Figure 5B). Collectively, the data indicate

269 that Chk1 activation by ATR intersects precisely with TopBP1 condensation. This suggests that

270 TopBP1 condensation operates as a switch-like mechanism that amplifies ATR activity above a

271 threshold required for Chk1 phosphorylation.

272 Of note, we observed the spontaneous activation of endogenous Chk1 in "Dignam and

273 Roeder" nuclear extracts after 10 minutes incubation at $37^{\circ} \mathrm{C}$ (Suppl. Figure 5A). The level of

274 phospho Chk1 (Ser345) signals was significantly higher than background signals observed when

275 reaction mixtures were incubated at $4^{\circ} \mathrm{C}$, or at $37^{\circ} \mathrm{C}$ in the presence of the ATR and mTOR

276 inhibitor ETP46464 (Suppl. Figure 5A), confirming that the reaction was the product of ATR

277 activity and occurred in vitro. In these experimental conditions, activation of endogenous Chk1

278 occurred in a purely endogenous protein extract. Neither recombinant TopBP1 protein nor

279 exogenous ATR-activating DNA structures were added to the extract. Phosphorylation of Chk1

280 was blocked when nuclear extracts were pre-incubated with ethidium bromide, which disrupts

281 protein-DNA interaction (Suppl. Figure 5B), indicating that endogenous DNA fragments present 
282 in the extracts are required for ATR activation. The spontaneous activation of endogenous ATR

283 in nuclear extracts is compatible with the hypothesis that exceeding a critical protein

284 concentration overcomes a kinetic barrier for the spontaneous assembly of functional

285 condensates that activate ATR/Chk1 signalling.

286 In Figures 5A-B we showed that TopBP1 induced phosphorylation of Chk1 on Ser345.

287 Once activated by ATR through Ser345 phosphorylation, Chk1 auto-phosphorylates on Ser296.

288 This step is required for Chk1 to induce downstream molecular events leading to cell cycle arrest

289 (Kasahara et al., 2010). Consistent with the hypothesis of opto-TopBP1 as functional assemblies,

290 light-induced TopBP1 condensation yielded Chk1 Ser296 phospho-signals, confirming that Chk1

291 is active (Suppl. Figure 5C). UCN-01, an inhibitor of Chk1, blocked Chk1 auto-phosphorylation

292 on Ser296, but had no major impact on Chk1 phosphorylation on Ser345 by ATR (Suppl. Figure

2935 C). This confirms that the Chk1 phospho Ser296 signal is a product of Chk1 activity. To

294 explore further the impact of TopBP1 condensation on TopBP1 interaction with partner proteins,

295 we took advantage from the optogenetic tool shown in Figure 1A. The TopBP1-mCherry-Cry2

296 used for light-induced foci formation is tagged at its N-terminus with TurboID, an optimized

297 biotin ligase that can biotinylated proteins within minutes (Branon et al., 2018). To detect ATR

298 activity within optogenetic TopBP1 condensates, we induced TopBP1 condensation by blue-light

299 illumination in the presence of biotin in the cell culture medium, and then purified biotinylated

300 proteins with streptavidin-coated beads. We enriched phospho ATR (Thr1989) from cells

301 expressing wild-type TopBP1 after optogenetic activation, specifically. We did not detect

302 phospho ATR signals in proximity of the condensation-defective mutant W1145R TopBP1

303 (Figure 5C). In fluorescence microscopy, TopBP1 condensates induced by optogenetic activation

304 co-localised with RAD9 and with phospho ATR (Suppl. Figure 5D-E). By contrast, Chk1 Ser345 
305 phospho-signals rarely co-localised with TopBP1 condensates per se, but were detected in cells

306 positive for TopBP1-mCherry condensates (Suppl. Figure 5F), consistent with Chk1 high

307 mobility (Liu et al., 2006). Chk1 is not retained physically at DNA damage sites, allowing signal

308 transmission from DNA damage sites to the rest of the cell (Liu et al., 2006). Super-resolution

309 STED imaging revealed phospho ATR (Thr1989) signals intertwined with TopBP1 nano-

310 condensates within TopBP1 clusters (Figure 5D). Collectively, these observations indicate that

311 TopBP1 condensates function as reaction hubs.

312 As $100 \%$ of optogenetic TopBP1 condensates were chromatin bound (Suppl. Figure 5E),

313 we analyzed the consequences of TopBP1 condensation on the progression of DNA replication

314 forks using a DNA fiber labelling approach. We labelled DNA replication tracks with two

315 consecutive pulses of the halogenated nucleotides IdU and CldU for 20 minutes each, and then

316 visualized DNA replication tracks by fluorescence microscopy. We induced TopBP1

317 condensation during the CldU pulse using two cycles of 3 minutes blue-light illumination, in

318 order to actuate and maintain TopBP1 foci during the 20 minutes labelling period (Figure 5E).

319 DNA replication tracks labelled in the presence of optogenetic TopBP1 condensates were shorter

320 than DNA replication tracks labelled in the absence of optogenetic activation. By contrast, blue-

321 light illumination did not alter the progression of replication forks in cells that express the

322 condensation defective mutant W1145R TopBP1. We conclude that TopBP1 condensates are

323 functional entities. Mechanistically, TopBP1 condensation triggers activation of ATR/Chk1

324 signalling.

325

326 Regulation of TopBP1 condensation 
327 TopBP1 is among the proteins that are most highly phosphorylated and sumoylated in response

328 to DNA replication stress (Munk et al., 2017). Post-translational modifications play a key role in

329 the regulation of the molecular forces that drive the formation of biomolecular condensates

330 (Soding et al., 2020). Since TopBP1 extensive capacity to self-assemble enables optogenetic

331 induction of TopBP1 nuclear condensates, we used this optogenetic system to explore the role of

332 phosphorylation in the formation of TopBP1 condensates. The activation of ATR/Chk1

333 signalling is dependent on TopBP1 phosphorylation (Burrows and Elledge, 2008; Hashimoto et

334 al., 2006; Yoo et al., 2007). Thus, we reasoned that the basal kinase activity of ATR, which is

335 independent of TopBP1 (Liu et al., 2011), may play a role in the condensation of TopBP1. To

336 test this, we pre-incubated cells with the ATR inhibitors VE-821 (Charrier et al., 2011), or ETP-

33746464 (Llona-Minguez et al., 2014), for 1 hour, and then exposed opto-TopBP1 expressing cells

338 to blue light for 3 minutes. In these experimental conditions, the formation of TopBP1

339 condensates was inhibited and the phosphorylation of Chk1 on the ATR site Ser345 was blocked

340 (Figure 6A + Suppl. Figure 6A). To explore further the role of ATR in TopBP1 condensation, we

341 mutated key amino acids in the AAD (Figure 6B). We substituted TopBP1 phenylalanine at

342 position 1071 with alanine. This substitution locates within a predicted coiled coil in TopBP1

343 AAD and destabilized TopBP1 interaction with ATR (Thada and Cortez, 2019). The F1071A

344 substitution abolished almost completely the optogenetic induction of TopBP1 condensates

345 (Figure 6C), confirming that TopBP1 interaction with ATR is required for TopBP1 higher order

346 assembly. In Xenopus Laevis, the phosphorylation of Ser1131 enhances the capacity of TopBP1

347 to activate ATR (Yoo et al., 2009). XTopBP1Ser1131 correspond to human Ser1138 TopBP1.

348 The substitution Ser1138A inhibited light-induced TopBP1 condensation, whereas the phospho

349 mimic Ser1138D stimulated TopBP1 condensation (Figure 6C). In blue-light exposed cells 
350 expressing F1071A TopBP1 or S1138A TopBP1, two mutations that impair TopBP1

351 condensation, the level of Chk1 phosphorylation on Ser345 was reduced in comparison with

352 cells expressing wild-type TopBP1 (Figure 6C, right panel). Furthermore, phospho Ser1138

353 TopBP1 and phospho Thr1989 ATR signals were barely detectable in streptavidin pulldowns

354 (Figure 6D). By contrast, the phosphomimetic substitution S1138D in TopBP1 yielded phospho

355 Ser345 Chk1 signals upon optogenetic activation (Figure 6C, right panel) and phospho Thr1989

356 ATR was enriched in proximity of TopBP1 (Figure 6D). Collectively, the data suggest an

357 amplification mechanism for activation of ATR/Chk1 signalling, whereby TopBP1

358 phosphorylation by ATR induces TopBP1 condensation, and TopBP1 condensation unleashes its

359 capacity to activate ATR. We propose that TopBP1 condensation is a molecular switch that

360 triggers checkpoint responses to DNA replication impediments. 


\section{Discussion}

362 In this study, we provide evidence that TopBP1 is a protein scaffold that can self-assemble

363 extensively to yield tight clusters of nano-condensates. TopBP1 condensation functions as a

364 molecular switch for ATR/Chk1 signalling. We show that TopBP1 condensation depends on

365 TopBP1 interaction with ATR, on the basal kinase activity of ATR and on the phosphorylation

366 of TopBP1. We obtained evidence that the phosphorylation of TopBP1 on serine 1138 is

367 essential for TopBP1 condensation. Thus, we propose a refined model of ATR activation (Figure

368 7): TopBP1 association with ATR yields a positive feedback loop, with TopBP1 condensation

369 acting as a mechanism of amplification promoting ATR signal transduction. In the early stages

370 of ATR signalling, ATR-ATRIP and TopBP1 congregates on RPA-coated single-stranded DNA,

371 the 9-1-1 complex is loaded at single to double strand DNA junctions and stabilizes TopBP1. In

372 later stages, the phosphorylation of TopBP1 induces its higher-order assembly into micron-sized

373 clusters of nano-condensates, where ATR activity is amplified and the checkpoint signal

374 transmitted. Our combined approach enabling the concomitant induction of TopBP1

375 condensation and the labelling of TopBP1 proximal proteins confirms that TopBP1 condensates

376 are the sites of ATR activation. The yeast homologue of ATR, Mec1, has been proposed to be

377 activated via an allosteric mechanism (Wang et al., 2017). We surmise that the compartmentation

378 of ATR signalling proteins creates a reaction hub where the probability of molecular interactions

379 required for ATR activation is increased. A TopBP1 clustering mechanism for ATR activation is

380 reminiscent of Ras nano-clusters, which are the sites of Ras effector recruitment and activation.

381 Ras clusters assemble transiently on the plasma membrane, function as high-gain amplifiers and

382 are critical for MAPK signal transduction (Prior et al., 2003; Tian et al., 2007). 
384 multiple weak and highly cooperative interactions of TopBP1 molecules. First, whether seeded

385 by hydroxyurea-induced replication stress or Cry2 oligomerisation, TopBP1 nuclear condensates

386 are dissolved upon addition of sorbitol or sucrose in the cell culture medium. These compounds

387 destabilize weak electrostatic interactions involved in protein phase separation. The aliphatic

388 alcohol hexanediol also dissolves TopBP1 condensates, suggesting that hydrophobic interactions

389 contribute to TopBP1 higher order assembly. Second, purified TopBP1 undergoes liquid-liquid

390 phase separation in physiologic salt and $\mathrm{pH}$, a characteristic feature of multivalent protein

391 scaffolds that underpin the formation of membrane-less compartments. It is noteworthy that

392 TopBP1 phase separation in vitro occurred not only in the presence of the crowding agent PEG,

393 but also in the complex environment of a nuclear extract, where multiple homotypic and

394 heterotypic interactions could influence the capacity of TopBP1 to self-assemble. Third, TopBP1

395 condensation was highly sensitive to key amino acid substitutions and post-translational

396 modifications within its intrinsically disordered ATR activation domain. These modifications

397 typically change the cooperative molecular forces that organize protein condensation. Point

398 mutations in the AAD that locally change the charge (W1145R, S1138D), the hydrophobicity

399 (F1071A) or the polarity (S1138A) modulate the growth of TopBP1 condensates induced by

400 optogenetic activation. Furthermore, the substitution W1145R in the AAD impaired the phase

401 separation of purified TopBP1 $1^{\mathrm{b}-6-8}$ in vitro. Last, our data indicate that the integrity of the

402 TopBP1 AAD is essential but not sufficient for the growth of TopBP1 nuclear condensates. In

403 isolation, the AAD of TopBP1 fused to Cry2 did not yield condensates, while the carboxy-

404 terminal half of TopBP1, which includes the AAD and BRCT6-8, assembled condensates. This 
405 indicates that the BRCT6-8 also contributes to TopBP1 higher-order assembly, consistent with

406 data showing that BRCT7/8 promotes TopBP1 oligomerisation (Chowdhury et al., 2014).

The assembly of complex heterogeneous biomolecules via multivalent, weak, dynamic

408 and cooperative interactions is emerging as a common principle of compartmentation in the

409 nucleus (Banani et al., 2017; Shin and Brangwynne, 2017; Soding et al., 2020). We expect the

410 rich diversity of molecular associations in live cells to yield diverse types of condensates with

411 distinct composition and biophysical properties. TopBP1 is a scaffold protein involved in

412 different pathways essentials for cell survival. In this study, we focused on its role in ATR

413 activation. TopBP1 condensates induced by optogenetic activation were variable in size and

414 fused occasionally, two characteristic features of non-stoichiometrically defined biomolecular

415 condensates. FRAP analysis indicates that the exchange of TopBP1 molecules outside and inside

416 TopBP1 compartments was unimpeded. High-resolution imaging by stimulated emission

417 depletion (STED) microscopy revealed that TopBP1 micron-sized foci detected by conventional

418 microscopy are made of clusters of nano-condensates, an organization that is likely to reflect the

419 role of chromatin in TopBP1 higher-order assembly. Consistent with this, our in vitro studies

420 suggest that long DNA polymers have major influence on the condensation of purified TopBP1.

421 The organization of TopBP1 into tight clusters of nano-condensates was common to both

422 recombinant TopBP1 condensates induced by optogenetic activation and endogenous TopBP1

423 condensates induced by DNA replication impediments. Interestingly, in mitosis, TopBP1 forms

424 filamentous structures that bridge MDC1 foci (Leimbacher et al., 2019), suggesting that specific

425 molecular associations dictate the organization of TopBP1 higher-order structures. The sub-

426 structural organization of TopBP1 condensates is reminiscent of 53BP1 foci, which consist in

427 nano-domains assembled into macro-domains (Ochs et al., 2019). Single-molecule localisation 
428 microscopy indicate that a 53BP1 repair focus is a composition of multiple clusters of 53BP1

429 molecules (McSwiggen et al., 2019), and its internal architecture is determined both by DNA

430 topology and protein-protein interactions that provide a structural scaffold (Ochs et al., 2019).

431 The function of TopBP1 condensation described here explains previous observations. In

432 Saccharomyces cerevisiae, artificial co-localisation of the 9-1-1 complex and Ddc2 ${ }^{\text {ATRIP- }}$

$433 \mathrm{Mec}^{\mathrm{ATR}}$ via tethering to an array of $256 \mathrm{LacO}$ repeats bypasses the requirement for DNA

434 damage to activate Mec1 ${ }^{\mathrm{ATR}}$ (Bonilla et al., 2008). In Schizosaccharomyces pombe, artificial

435 tethering of either one of $\operatorname{Rad} 3^{\mathrm{ATR}}, \mathrm{RAD}^{\mathrm{TopBP} 1}$ or RAD9 to a LacO array triggers a checkpoint

436 response that utilizes the endogenous proteins (Lin et al., 2012). The LacO recruited Rad3 ${ }^{\text {ATR }}$

437 must phosphorylate endogenous RAD9 to promote $\operatorname{Rad} 4^{\mathrm{TopBP} 1}$ recruitment and activate the

438 checkpoint (Lin et al., 2012). Furthermore, TopBP1 activates ATR in vitro and in cells when

439 artificially tethered to DNA (Lindsey-Boltz and Sancar, 2011). Based on the findings described

440 here, we surmise that the artificial tethering of checkpoint proteins to LacO arrays is nucleating

441 the condensation of endogenous proteins, which switches on checkpoint signalling.

442 The transient and reversible nature of the molecular forces that underpin the formation of

443 functional TopBP1 nuclear condensates appears well adapted to cellular regulation and optimal

444 responsivity to DNA replication impediments, as opposed to the stable interaction of proteins

445 that characterize molecular machines with defined stoichiometry. The formation of functional

446 micron-sized condensates through the regulated self-assembly of multivalent protein scaffolds

447 may represent a fundamental principle underlying the formation of functional nuclear foci in

448 response to DNA damage. 


\section{Acknowledgments}

450 We thank all members of the laboratory, Olivier Ganier and Pierre-Henri Gaillard for their

451 critical reading of the manuscript. We are grateful to Lee Zou for the cDNA encoding TopBP1,

452 Brian Raught for pCDNA5_FRT-TO_FlagBirA*, Clifford P Brangwynne for the cDNA

453 encoding Cry2, Juhani E. Syväoja for the U-2-OS cell lines expressing eGFP-TopBP1 and

454 Simon Alberti for technical information. This work was supported by MSD Avenir, by la

455 Fondation ARC pour la recherche sur le cancer (PGA1 RF20180206787), and by the SIRIC

456 Montpellier Cancer (grant INCa_Inserm_DGOS_12553).

\section{Author contribution:}

459 Conceptualization: C.F., J.B. and A.C; Methodology: C.F., J.B. and A.C.; Investigation: C.F., 460 A.P., S.V., E.A., M.L., M-P.B., S.U. and J.B.; Data curation: C.F., A.P. J.B and A.C. Writing-

461 original draft: C.F., J.B. and A.C. Writing-Review \& Editing: C.F., A.P., J.B. and A.C.

462 Supervision: J.B. and A.C. Project Administration: A.C. Funding Acquisition: A.C. 


\section{Methods}

\section{Resources tables:}

\begin{tabular}{|c|c|}
\hline Primers & Sequence \\
\hline Primer $1(\mathrm{Fw})$ & TTGGCGCGCCAtccagaaatgacaaagaaccgt \\
\hline Primer 2 (Rev) & TTTTCCTTTTGCGGCCGCttagtgtactctaggtcgtttg \\
\hline Primer 3 (untisense) & GTAGGGTCATCCCTAATGATCTGTTCATTTTGGGAAGGC \\
\hline Primer $4(\mathrm{Fw})$ & GGGGTACCATCGATGCTAGCatggtgtctaaaggcgagga \\
\hline Primer 5 (Rev) & GGCGCGCCtcagtcacgcatgttgcaggt \\
\hline Primer $6(\mathrm{Fw})$ & GGGGTACCACCATGctttctgccagccetca \\
\hline Primer 7 (Rev) & CTAGCTAGCgtgtactctaggtcgtttgatt \\
\hline Primer $8(\mathrm{Fw})$ & gggCTTAAGgccaccATGaaagacaatactgtgcctctg \\
\hline Primer 9 (Rev) & cggGGTACCCACGTGcttttcggcagaccgcaga \\
\hline Primer $10(\mathrm{Fw})$ & gggCACGTGtccagaaatgacaaagaaccg \\
\hline Primer $11(\mathrm{Rev})$ & cggGGTACCgtgtactctaggtcgtttgat \\
\hline Primer 12 (sense) & gagatgagagagaacgctcagaagcagttacag \\
\hline Primer 13 (sense) & gtcaacacagagcctgcccaaaatgaacagatc \\
\hline Primer 14 (sense) & gtcaacacagagcctgaccaaaatgaacagatc \\
\hline Primer $15(\mathrm{Fw})$ & CTAGCGTTTAAACTTAAGATGgctgtgtcttcaacaaaggatg \\
\hline Primer 16 (Rev) & cctaCATGGTACCatcatgtaaaggcttttgaaaagg \\
\hline Primer $17(\mathrm{Fw})$ & tacacGGTACCATGaggaaagctcctacag \\
\hline Primer 18 (Rev) & caccatGCTAGCtctaggtcgtttgatttta \\
\hline Primer $19(\mathrm{Fw})$ & AGCTTGGTACCATGAGTCGGCGAAGGAAACATGATG \\
\hline Primer $20(\mathrm{Fw})$ & ccgaaaagCACGTGGGTACCAAGTGCCTGGTCACGGG \\
\hline Primer $21(\mathrm{Rev})$ & atGCTAGCATCGATGGTACCGCCTTCACCCTCACTGTCTTCC \\
\hline Primer 22 (Rev) & caccatGCTAGCAAGAAATGAAGTGGGAGCTGC \\
\hline Primer $23(\mathrm{Fw})$ & tacacctggatacaccatcaaaattcctgtccaaggacaaac \\
\hline Primer 24 (Rev) & CTTTGGTGTTTCATAtgagagtagtcgactattacagagccg \\
\hline Primer 25 ( Fw ) & agtcgactactctcaTATGAAACACCAAAGAGAGCGCTGA \\
\hline Primer 26 (Rev) & agcaatttctgaatcTGACCACATCTGTATATTATCTTTATAATTACTTAAAATCTCTGTTTCT \\
\hline
\end{tabular}




\section{6}

\begin{tabular}{|l|r|}
\hline Primer $27(\mathrm{Fw})$ & ATACAGATGTGGTCAgattcagaaattgctaaacaggctgtct \\
\hline Primer $28(\mathrm{Rev})$ & ttcetgcacgaagtgtccagcagtcetgcaggcttcaaggta \\
\hline
\end{tabular}

\begin{tabular}{|c|c|c|}
\hline Plasmids & Expression in & Origin \\
\hline pOG44 (Vector for expression of the Flp recombinase) & Human cells & ThermoFisher Scientific \\
\hline 3xHA-TurboID-NLS_pCDNA3 & Human cells & Addgene ( \#107171) \\
\hline pCDNA5_FRT-TO_Flag-BirA* & Human cells & Raught's lab \\
\hline pHR-mCherry-Cry2 & Human cells & Brangwynne's lab \\
\hline pCDNA5_FRT-TO_GFP-ETAA1 & Human cells & Mailand's lab \\
\hline pDONR223-RAD9A & Human cells & MGC \\
\hline pCDNA5_FRT-TO_TurboID-mCherry-Cry2 (opto-module) & Human cells & This study \\
\hline pCDNA5_FRT-TO_TurboID-TopBP1WT-mCherry-Cry2 & Human cells & This study \\
\hline pCDNA5_FRT-TO_TurboID-TopBP1W1145R-mCherry-Cry2 & Human cells & This study \\
\hline pCDNA5_FRT-TO_TurboID-ETAA1-mCherry-Cry2 & Human cells & This study \\
\hline pCDNA5_FRT-TO_TurboID-TopBP1 ${ }^{\text {ETAA1-AAD }}$-mCherry-Cry2 & Human cells & This study \\
\hline pCDNA5_FRT-TO_TurboID-RAD9A-mCherry-Cry2 & Human cells & This study \\
\hline pFastBac1_6His_MBP_TopBP1WT ${ }^{\mathrm{b} 6-8}$-mGFP & Insect cells & GeneScript \\
\hline pFastBac1_6His_MBP_TopBP1W1145R ${ }^{\mathrm{b} 6-8}$-mGFP & Insect cells & GeneScript \\
\hline pFastBac1_6His_MBP_TopBP1WT ${ }^{\mathrm{b} 6-8}$-mRFP & Insect cells & GeneScript \\
\hline pFastBac1_6His_MBP_TopBP1W1145R ${ }^{\mathrm{b} 6-8}$-mRFP & Insect cells & GeneScript \\
\hline pCDNA5_FRT-TO_Flag-BirA*-TopBP1WT & Human cells & This study \\
\hline pCDNA5_FRT-TO_Flag-BirA*-TopBP1W1145R & Human cells & This study \\
\hline pCDNA5_FRT-TO_mCherry-Cry2 (opto-module\#) & Human cells & This study \\
\hline pCDNA5_FRT-TO_TopBP1WT ${ }^{\mathrm{b} 6-8}$-mCherry-Cry2 & Human cells & This study \\
\hline pCDNA5_FRT-TO_TopBP1W1145R ${ }^{\mathrm{b} 6-8}$-mCherry-Cry2 & Human cells & This study \\
\hline pCDNA5_FRT-TO_AAD-NLS ${ }^{\text {TopBP1 }}$-mCherry-Cry2 & Human cells & This study \\
\hline pCDNA5_FRT-TO_TurboID-TopBP1F1071A-mCherry-Cry2 & Human cells & This study \\
\hline pCDNA5_FRT-TO_TurboID-TopBP1S1138A-mCherry-Cry2 & Human cells & This study \\
\hline pCDNA5_FRT-TO_TurboID-TopBP1S1138D-mCherry-Cry2 & Human cells & This study \\
\hline
\end{tabular}


Antibodies:

\begin{tabular}{|c|c|c|}
\hline Primary antibodies & Reference & Use \\
\hline ATR & Bethyl/euromedex, A300-137A & WB \\
\hline BRCA1 (C-20) & Santa Cruz, sc-642 & WB \\
\hline Chk1 & Santa Cruz, sc- 8408 & WB \\
\hline FANCJ/BRIP1 & Novus Biologicals, NB100-416 & WB \\
\hline Flag-M2 & Sigma, F1804 & IF \\
\hline mCherry & Elabscience, E-AB-20087 & IF/WB \\
\hline MRE11 & Novus Biologicals, NB100-142 & WB \\
\hline NOL11 & Sigma, HPA022010 & WB \\
\hline pATR (T1989) & Euromedex, GTX128145 & IF/WB \\
\hline pChk1 (Ser296) & Cell Signalling Technology, 2349 & WB \\
\hline pChk1 (Ser345) & Cell Signalling Technology, 2348L & IF/WB \\
\hline pTopBP1 (Ser1138) & Interchim, orb140434 & WB \\
\hline Streptavidin, Alexa Fluor 488 conjugate & Invitrogen, S32354 & IF \\
\hline Tubulin (B-5-1-2) & Sigma, T5168 & WB \\
\hline TopBP1 & Euromedex, A300-111A & $\mathrm{IF} / \mathrm{WB}$ \\
\hline
\end{tabular}

\begin{tabular}{|l|c|c|}
\hline Secondary antibodies & Reference & Use \\
\hline Alexa Fluor 546 goat anti-mouse IgG & Invitrogen, A11030 & IF 1/500 \\
\hline Alexa Fluor 546 goat anti-rabbit IgG & Invitrogen, A11010 & IF 1/500 \\
\hline Alexa Fluor 488 goat anti-mouse IgG2b & Invitrogen, A21141 & IF 1/600 \\
\hline Alexa Fluor 488 goat anti-rabbit IgG & Invitrogen, A11008 \\
\hline Anti-rabbit IgG Atto-647N & Sigma, 40839 $1 / 600$ \\
\hline Anti-mouse IgG Atto-647N & Sigma, 50185 \\
\hline Anti-mouse IgG Abberior STAR 580 & Sigma, 38377 $1 / 600$ \\
\hline Anti-mouse IgG, HRP linked Antibody & Cell Signalling, 7076 & WB 1/5000 \\
\hline Anti-rabbit IgG, HRP linked Antibody & Cell Signalling, 7074 & WB 1/5000 \\
\hline
\end{tabular}




\section{Cell lines.}

$473 \underline{\text { Flp-In }{ }^{\mathrm{TM} T} \text {-REx }} \mathrm{TM}^{\mathrm{TM}} 293$ and $\underline{H E K 293}$ cell lines were grown under standard conditions $\left(37^{\circ} \mathrm{C}, 5 \%\right.$

$474 \mathrm{CO}_{2}$ ) in Dulbecco's modified Eagle's medium (Merck-Sigma-Aldrich, D5796). For Flp-In ${ }^{\mathrm{TM} T}$ -

$475 R E x^{\mathrm{TM}} 293$ the medium was supplemented with $10 \%$ fetal bovine serum (FBS), $100 \mu \mathrm{g} / \mathrm{ml}$ Zeocin

476 and $15 \mu \mathrm{g} / \mathrm{ml}$ Blasticidin. Flp-In ${ }^{\mathrm{TM} T}$-REx ${ }^{\mathrm{TM}} 293$ transfected cells were selected and maintained

477 with $15 \mu \mathrm{g} / \mathrm{mL}$ Blasticidin and $150 \mu \mathrm{g} / \mathrm{mL}$ Hygromycin.

$478 \underline{U}-2-O S$ Tet-On cell lines expressing eGFP-TopBP1 WT were grown under standard conditions

$479\left(37^{\circ} \mathrm{C}, \quad 5 \% \quad \mathrm{CO}_{2}\right)$ in modified McCoy's 5a medium (Merck-Sigma-Aldrich, M9309)

480 supplemented with $10 \%$ fetal bovine serum (FBS), $100 \mu \mathrm{g} / \mathrm{ml}$ of Hygromycin and $200 \mu \mathrm{g} / \mathrm{ml}$ of 481 G418 as selective antibiotics (Sokka et al., 2015). Expression of eGFP-TopBP1 WT was induced 482 with $1 \mu \mathrm{g} / \mathrm{ml}$ of doxycycline for 24 hours.

484 Sf9 insect cells culture conditions. Sf9 cells were grown in EX-CELL® 420 Serum-free 485 medium (Sigma-Aldrich, 14420C). Cells were maintained between $2 \times 10^{6}$ and $1 \times 10^{7}$ cells $/ \mathrm{ml}$ at $48628^{\circ} \mathrm{C}$ in flasks (agitation $140 \mathrm{rpm}$ ).

488 Plasmid constructs.

489 For pCDNA5_FRT-TO_Flag-BirA*-TopBP1 WT or W1145R, TopBP1 full-length cDNA (a 490 kind gift from Lee Zou) was amplified by PCR with primers 1 and 2 using Phusion® High491 Fidelity DNA Polymerase (New England Biolabs, CM0530). The forward and reverse primers 492 contain AscI and NotI sites, respectively. The amplified PCR was inserted into the 
pCDNA5_FRT-TO_Flag-BirA* (a kind gift from Biran Raught) linearised with AscI/NotI

494 digestion.

495 For pCDNA5_FRT-TO_mCherry-Cry2 (opto-module\#), the Flag-BirA* fragment was deleted

496 from the pCDNA5_FRT-TO_FlagBirA* using KpnI/AscI enzymes and replaced with the

497 mCherry-Cry2 fragment amplified by PCR from the plasmid pHR-mCherry-Cry2 (a kind gift

498 from Brangwynne's lab) with primers 4 and 5. In the second step, TopBP1 amino acids 884 to

4991522 fragment (BRCT6-AAD-BRCT7-8 WT or W1145R) was amplified with primers 6 and 7 ,

500 digested with KpnI/NheI and inserted into pCDNA5_FRT-TO_mCherry-Cry2 to produce opto-

501 TopBP ${ }^{\mathrm{b} 6-8} \mathrm{WT}$ and opto-TopBP $1{ }^{\mathrm{b} 6-8} \mathrm{~W} 1145 \mathrm{R}$. For pCDNA5_FRT-TO_TurboID-mCherry-Cry2

502 construction (opto-module), the TurboID fragment was amplified by PCR from the 3xHA-

503 TurboID-NLS_pCDNA3 plasmid (Addgene \#107171) with primers 8 and 9, digested with

504 AflII/KpnI enzymes and inserted into the opto-module\# to produce the opto-module. In the

505 second step, TopBP1 WT and W1145R were amplified by PCR with primers 10 and 11, digested

506 with PmlI/KpnI enzymes and inserted into the opto-module construct. Mutations in the AAD of

507 TopBP1 were generated using the "QuickChange Multi Site-directed mutagenesis kit" (Agilent

508 technologies, C200515): W1145R with primer 3, F1071A with primer 12, S1138A with primer

509 13, S1138D with primer 14. pCDNA5_FRT-TO_TurboID-AAD-NLS ${ }^{\text {TopBP1}}$-mCherry-Cry2 was

510 generated by PCR amplification of TopBP1 AAD (primers 15-16) and TopBP1 NLS ( primers

511 17-18). Fragments were inserted in 2 steps into the opto-module digested with AflII/KpnI

512 (TopBP1 AAD) and KpnI/NheI (TopBP1 NLS) enzymes respectively. pCDNA5_FRT-

513 TO_TurboID-ETAA1-mCherry-Cry2 was generated by PCR amplification of ETAA1 with

514 primers 19 and 20 on pCDNA5_FRT-TO_GFP-ETAA1 (a kind gift from Mailand's lab) and

515 insertion of the fragments into the opto-module digested with KpnI/NheI enzymes. 
pCDNA5_FRT-TO_TurboID-RAD9A-mCherry-Cry2 was generated by PCR amplification of

517 RAD9 with primers 21 and 22 on pDONR223-RAD9A (obtained through MGC Montpellier

518 Genetic Collections) and fragment was cloned into the KpnI-digested opto-module following the

519 In-Fusion HD Cloning Kit protocol. The plasmid with the chimeric constructs of TopBP1

520 carrying the AAD of ETAA1 (pCDNA5_FRT-TO_TurboID-TopBP1 ${ }^{\text {ETAA1-AAD }}$-mCherry-Cry2)

521 was generated using the NEBuilder HiFi DNA assembly Master Mix (New England Biolabs,

522 E2621L). This kit was used to assemble multiple DNA fragments with 30 bp-overlap and replace

5231530 bp inside TopBP1. DNA fragments were produced by PCR: oligos 23-24 were used to

524 amplify TopBP1 sequence before the AAD (PCR on any plasmid containing full-length

525 TopBP1), oligos 25-26 were specific for the AAD of ETAA1 (PCR on pCDNA5_FRT-TO

526 _GFP-ETAA1) and oligos 27-28 were specific for TopBP1 portion after the AAD (PCR on any

527 plasmid containing full-length TopBP1). To obtain the chimeric constructs, PCR products were

528 assembled according to the manufacturer's instruction and ligated into pCDNA5_FRT-

529 TO_TurboID-TopBP1-mCherry-Cry2 digested with EcoNI/SbfI.

530 pFastBac1 plasmids containing WT and W1145R BRCT 6 to 8 fragments of TopBP1 were

531 synthesized by GeneScript after codon optimization for expression in insect cells (sequence

532 available upon request), and sub-cloned into the 6His-MBP_3C_MCS_TEV_mRFP and 6His-

533 MBP_3C_MCS_TEV_mGFP cassette using the restriction sites EcoRI/KpnI.

\section{Western Blotting.}

536 Whole cell extracts were lysed with 1X Laemmli Sample buffer (Biorad, C161-0737) and heated

$5375 \mathrm{~min}$ at $95^{\circ} \mathrm{C}$. Cell extracts were resolved using pre-cast SDS-PAGE (7.5\% and 10\%) from

538 BioRad and transferred to nitrocellulose membrane using a transfer apparatus according to the 
manufacturer's instructions (BioRad). Membranes were saturated with $10 \%$ non-fat milk diluted

540 in TBS- $0.2 \%$ Tween 20 (TBS-T), incubated with primary antibodies overnight at $4{ }^{\circ} \mathrm{C}$ and with

541 anti-mouse-HRP or anti-rabbit-HRP secondary antibodies for $1 \mathrm{~h}$. Blots were developed with

542 ECL according to the manufacturer's instructions.

544 Affinity capture of biotinylated proteins: BioID.

545 Flp-In ${ }^{T M} T-R E x^{T M} 293$ cell lines stably transfected with Flag-BirA*-TopBP1 WT or W1145R 546 grown to $75 \%$ confluence were incubated with $1 \mu \mathrm{g} / \mathrm{ml}$ of doxycycline (Clontech, 631311) for

$54716 \mathrm{~h}$ and with $50 \mu \mathrm{M}$ biotin for 3 or 16 hours. Cells were washed with PBS and lysed with lysis 548 buffer $(50 \mathrm{mM}$ Tris-HCl pH 7.5, 150mM NaCl, 1mM EDTA, 1mM EGTA, 1\% NP-40, 0.2\%

549 SDS, 0.5\% Sodium deoxycholate) supplemented with 1X complete protease inhibitor (Roche)

550 and $250 \mathrm{U}$ benzonase (Sigma, CE1014). Lysed cells were incubated on a rotating wheel for $1 \mathrm{~h}$ at

$5514{ }^{\circ} \mathrm{C}$ prior sonication on ice (40\% amplitude, 3 cycles $10 \mathrm{sec}$ sonication- 2 sec resting). After

$55230 \mathrm{~min}$ centrifugation $(7750 \mathrm{rcf})$ at $4^{\circ} \mathrm{C}$, the cleared supernatant was transferred to a new tube and

553 total protein concentration was determined by Bradford protein assay (BioRad, C500-0205). For

554 each condition, $300 \mu \mathrm{g}$ of proteins were incubated with $30 \mu \mathrm{l}$ of Streptavidin-Agarose beads

555 (Sigma, CS1638) on a rotating wheel at $4^{\circ} \mathrm{C}$ for $3 \mathrm{hr}$. After $1 \mathrm{~min}$ centrifugation (400 rcf), beads

556 were washed, successively, with $1 \mathrm{ml}$ of lysis buffer, $1 \mathrm{ml}$ wash buffer $1\left(2 \%\right.$ SDS in $\left.\mathrm{H}_{2} \mathrm{O}\right), 1 \mathrm{ml}$

557 wash buffer $2(0.2 \%$ sodium deoxycholate, $1 \%$ Triton $\mathrm{X}-100,500 \mathrm{mM} \mathrm{NaCl}, 1 \mathrm{mM}$ EDTA, and

$55850 \mathrm{mM}$ Hepes $\mathrm{pH} 7.5), 1 \mathrm{ml}$ wash buffer $3(250 \mathrm{mM} \mathrm{LiCl}, 0.5 \%$ NP-40, $0.5 \%$ sodium

559 deoxycholate, $1 \mathrm{mM}$ EDTA, $500 \mathrm{mM} \mathrm{NaCl}$ and $10 \mathrm{mM}$ Tris $\mathrm{pH} 8)$ and $1 \mathrm{ml}$ wash buffer $4(50 \mathrm{mM}$

560 Tris $\mathrm{pH} 7.5$ and $50 \mathrm{mM} \mathrm{NaCl}$ ). Bound proteins were eluted from the magnetic beads using $80 \mu \mathrm{l}$

561 of $2 \mathrm{X}$ Laemmli Sample buffer and incubated at $95^{\circ} \mathrm{C}$ for $10 \mathrm{~min} .10 \%$ of the sample was used for

562 Western blot analysis. For the Flp-In ${ }^{T M} T-R E x^{T M} 293$ cell lines stably transfected with the 
563 doxycycline-inducible TurboID-TopBP1WT-mCherry-Cry2 or the mutated forms of TopBP1,

564 cells were simultaneously incubated with $500 \mu \mathrm{M}$ of biotin and exposed to blue light for $10 \mathrm{~min}$ of

565 light-dark cycles (4s light followed by 30s dark). Biotin proximity labelling of light-induced

566 TopBP1 partners were pulled-down using streptavidin-coated beads as described before and

567 probed by immunoblotting to detect proteins that are associated with TopBP1 clusters, in

568 absence of DNA damage.

570 Mass spectrometry.

571 Sample digestion was essentially performed as described (Shevchenko et al., 2006). Briefly,

572 proteins were loaded on a SDS-PAGE (BioRad, 456-1034) and, after short migration, a single

573 band was excised. Proteins in the excised band were digested with Trypsin (Promega, V5280).

574 The resulting peptides were analyzed online by nano-flow HPLC-nanoelectrospray ionization

575 using a Qexactive HFX mass spectrometer (Thermo Fisher Scientific) coupled to a nano-LC

576 system (Thermo Fisher Scientific, U3000-RSLC). Desalting and preconcentration of samples

577 were performed online on a Pepmap® precolumn $(0.3$ x 10mm; Fisher Scientific, 164568). A

578 gradient consisting of $0 \%$ to $40 \% \mathrm{~B}$ in A (A: $0.1 \%$ formic acid [Fisher Scientific, A117], 6\%

579 acetonitrile [Fisher Scientific, A955], in $\mathrm{H}_{2} \mathrm{O}$ [Fisher Scientific, W6], and B: 0.1\% formic acid in

$58080 \%$ acetonitrile) for $120 \mathrm{~min}$ at $300 \mathrm{nl} / \mathrm{min}$ was used to elute peptides from the capillary reverse-

581 phase column $(0.075$ x 250mm, Pepmap®, Fisher Scientific, 164941). Data were acquired using

582 the Xcalibur software (version 4.0). A cycle of one full-scan mass spectrum $(375-1,500 \mathrm{~m} / \mathrm{z})$ at a

583 resolution of 60000 (at 200m/z) followed by 12 data-dependent MS/MS spectra (at a resolution

584 of 30000 , isolation window $1.2 \mathrm{~m} / \mathrm{z}$ ) was repeated continuously throughout the nanoLC

585 separation. Raw data analysis was performed using the MaxQuant software (version 1.5.5.1) 
586 with standard settings. Used database consist of Human entries from Uniprot (reference

587 proteome UniProt 2018_09) and 250 contaminants (MaxQuant contaminant database). Graphical

588 representation and statistical analysis were performed using Perseus (version 1.6.1.1). A standard

589 t-test was used to evaluate protein abundance difference between samples.

591 TopBP1 expression and purification.

592 Plasmids for protein expression in insect cells using baculoviruses are listed in Table Plasmids.

593 For the production of bacmids, 50ng of pFastbac plasmids were transformed into MultiBac

594 DH10 cells (Invitrogen, 10361-012) and positive clones were selected on LB Ampicilin

$595(100 \mu \mathrm{g} / \mathrm{ml})$ plates supplemented with $40 \mu \mathrm{g} / \mathrm{ml}$ IPTG, $100 \mu \mathrm{g} / \mathrm{ml} \mathrm{XGal} \mathrm{and} 7 \mu \mathrm{g} / \mathrm{ml}$ Gentamycin.

596 Blue colonies were screened for the presence of inserts by colony PCR using pUC/M13 Forward

597 and Reverse oligos (Bac-to-Bac ${ }^{\circledR}$ Baculoviruses Expression System_invitrogen user guide). To

598 generate baculoviruses, $12 \times 10^{6} \mathrm{Sf} 9$ cells $(1 \mathrm{ml})$ were transfected with $5 \mu \mathrm{g}$ of purified bacmid

599 using $15 \mu \mathrm{l}$ of Cellfectin ${ }^{\mathrm{TM}}$ (Invitrogen, P/N 58760). After 5h incubation, 9ml of medium were

600 added to Sf9 cells and cultures were incubated for 2.5 days. The supernatant (P1) was collected

601 by centrifugation ( $400 \mathrm{rcf} 10 \mathrm{~min}$ ) and a $1 / 100$ dilution was used to infect $4 \times 10^{6}$ cells $/ \mathrm{ml} \mathrm{Sf9}$

602 cells. Cells were incubated for 2 days and the supernatant (P2) was collected by centrifugation.

603 Expression of fluorescent proteins was verified by Western Blotting and microscopy. For protein

604 expression, a 1/10 dilution of freshly prepared P2 was added to $2 \times 10^{6}$ cell/ml Sf9 culture and

605 incubated for 48 to 72 hours.

606 Infected cells were collected and lysed mechanically using a HTU-DIGI-French-Press (10000

607 PSI) in $15 \mathrm{X}$ packed cell weight hypertonic lysis buffer $\left(50 \mathrm{mM} \mathrm{Na} 2 \mathrm{H} / \mathrm{NaH}_{2} \mathrm{PO}_{4} \mathrm{pH} 8.0,500 \mathrm{mM}\right.$

$608 \mathrm{NaCl}, 1 \%$ glycerol, $0.1 \%$ CHAPS) supplemented with protease inhibitors. Lysate was clarified 
609 by centrifugation $\left(7750 \mathrm{rcf}, 40 \mathrm{~min}, 4^{\circ} \mathrm{C}\right)$, filtered and loaded on a $5 \mathrm{ml}$ His Trap HP column (GE

610 Healthcare, 71-5027-68 AF) equilibrated with 5CV (Column Volumes) of buffer A (lysis buffer

$611+5 \mathrm{mM}$ imidazole). The column was washed with $5 \mathrm{CV}$ of buffer A and TopBP1 ${ }^{\mathrm{b} 6-8}$ was eluted

612 stepwise using 5CV of buffer A + 30mM, 50mM, 75mM, 125mM and 500mM imidazole. Peak

613 TopBP $^{\mathrm{b} 6-8}$ fractions (eluted with $50 \mathrm{mM}$ and $75 \mathrm{mM}$ imidazole) were desalted (HiTrap ${ }^{\mathrm{TM}}$ or

614 HiPrep 26/10, GE Healthcare) in physiological buffer $\left(10 \mathrm{mM} \mathrm{Na} 2 \mathrm{H} / \mathrm{NaH}_{2} \mathrm{PO}_{4} \mathrm{pH} 7.6,150 \mathrm{mM}\right.$

615 KOAc, 0.1mM MgOAc, 0.5mM DTT, $2.5 \%$ glycerol), snap frozen and stored at $-80^{\circ} \mathrm{C}$. Protein

616 concentration was estimated by stainfree gel quantification using Image Lab Software.

618 Nuclear extract preparation.

619 Nuclear extracts were prepared as previously described (Vidal-Eychenie et al., 2013). HeLa S3

620 cells were grown to $\leq 80 \%$ confluence, collected by scrapping, centrifuged ( $\left.200 \mathrm{rcf}, 3 \mathrm{~min}, 4^{\circ} \mathrm{C}\right)$

621 and washed twice in PBS 1X. Cell pellet was incubated on ice for 5min in $5 \mathrm{X}$ packed cell

622 volume of hypotonic buffer A (10mM Hepes-KOH pH 7.9, $10 \mathrm{mM} \mathrm{KCl,} 1.5 \mathrm{mM} \mathrm{MgCl}, 0.5 \mathrm{mM}$

623 DTT, 0.5mM PMSF) supplemented with protease (complete, EDTA free; Roche, 31075800) and

624 phosphatase inhibitors (Fisher Scientific). Cells were then spun down (500 rcf, 5min), suspended

625 in $2 \mathrm{X}$ packed cell volume of buffer A and lysed by dounce homogenization using a tight-fitting

626 pestle. Nuclei were collected by centrifugation (4000 rcf) for $5 \mathrm{~min}$ at $4{ }^{\circ} \mathrm{C}$, extracted in one

627 nuclei pellet volume of buffer $\mathrm{C}\left(20 \mathrm{mM}\right.$ Hepes-KOH pH 7.9, 600mM KCl, $1.5 \mathrm{mM} \mathrm{MgCl}_{2}$,

$6280.2 \mathrm{mM}$ EDTA, 25\% glycerol, 0.5mM DTT, 0.5mM PMSF) supplemented with cocktails of

629 protease and phosphatase inhibitors, and mixed on a rotating wheel at $4^{\circ} \mathrm{C}$ for $30 \mathrm{~min}$. Nuclear

630 extracts (supernatants) were recovered by centrifugation $\left(16000 \mathrm{rcf}, 15 \mathrm{~min}, 4^{\circ} \mathrm{C}\right)$ and dialyzed

631 using Slide-A-Lyzer Dialysis Cassettes (3,500-D protein molecular weight cutoff; Fisher 
632 Scientific, 68035) against buffer D (20mM Hepes-KOH pH 7.9, 100mM KCl, 0.2mM EDTA,

$63320 \%$ glycerol, $0.5 \mathrm{mM}$ DTT, and 0.5mM PMSF). Dialyzed nuclear extracts were centrifuged

634 (100000 $\mathrm{rcf}, 30 \mathrm{~min}, 4^{\circ} \mathrm{C}$ ) to eliminate residual precipitates. The protein concentration of the clear

635 supernatant was determined by Bradford (BioRad, C500-0205) protein assay, and aliquots were

636 snap frozen and stored at $-80^{\circ} \mathrm{C}$. Important to note that the Dignam \& Roeder extract preparation

637 contain DNA fragments after centrifugation.

639 TopBP1 phase separation assay.

640 Phase separation of purified TopBP1 $1^{\text {b6-8 }}$ was performed in physiological buffer C (10mM

$641 \mathrm{Na}_{2} \mathrm{H} / \mathrm{NaH}_{2} \mathrm{PO}_{4} \mathrm{pH}$ 7.6, 150mM KOAc, 0.1mM MgOAc, 0.5mM DTT, 2.5\% Glycerol). Purified

642 TopBP1 ${ }^{\mathrm{b6-8}}$ WT and W1145R were digested with PreScission 3C enzyme (GenScript, Z03092-

643500 ) for $3 \mathrm{~h}$ at $16^{\circ} \mathrm{C}$ to remove the 6-His and MBP tag before phase separation in reaction

644 mixtures in buffer $\mathrm{C}$ containing $10 \mu \mathrm{M}$ of WT or W1145R TopBP1 ${ }^{\text {b6-8 }}$-GFP and 2\% of PEG4000

645 (Merck-Sigma-Aldrich, 95904). Reaction mixtures were mixed by gently tapping the Eppendorf.

646 TopBP1 droplet permeability assay was performed in two steps: first, TopBP $1^{\text {b6-8 }}$-GFP droplets

647 were formed in reaction mixtures containing $10 \mu \mathrm{M}$ of WT TopBP $1^{\text {b6-8 }}$-GFP supplemented with

$6482 \%$ PEG in physiological buffer $\mathrm{C}$, and then we added either $1 \mu \mathrm{M}$ of $\mathrm{WT}$ or W1145R TopBP1 ${ }^{\mathrm{b}-}$

$649{ }^{8}$-RFP or StuI/SacII digested pX174 RFII DNA (300nM), as indicated. To study the role of DNA

650 in the organization of TopBP1 condensates, we incubated $2.5 \mu \mathrm{M}$ of WT TopBP1 ${ }^{\text {b6-8 }}$ with $40 \mathrm{ng}$

$6512.9 \mathrm{~kb}$ circular DNA plasmid DNA at $37^{\circ} \mathrm{C}$ during $10 \mathrm{~min}$ in physiological buffer C. The ratio

652 purified TopBP1:DNA was decided according to Choi JH et al., 2008. Samples were imaged on

653 a LSM780 confocal microscope (Leica, Germany) using a 63x oil immersion objective (N.A.

654 1.4). DAPI and GFP fluorescence were excited at 405 and 488nm respectively, and emitted 
655 fluorescence were collected sequentially at 415-460nm and 500-550nm respectively. The pinhole

656 size was set to 1 Airy unit.

657 TopBP1 phase separation in human protein extracts was performed in reaction mixtures 658 containing $0.2 \mu \mathrm{g} / \mu \mathrm{l}$ of human nuclear extract, $2 \mu \mathrm{M}$ of WT or W1145R TopBP ${ }^{\mathrm{b} 6-8}$-GFP in ATR

659 activation buffer $(10 \mathrm{mM}$ Hepes-KOH pH 7.6, $50 \mathrm{mM} \mathrm{KCl}, 0.1 \mathrm{mM} \mathrm{MgCl} 2,1 \mathrm{mM}$ PMSF, $0.5 \mathrm{mM}$

660 DTT, $1 \mathrm{mM}$ ATP, $10 \mu \mathrm{g} / \mathrm{ml}$ creatine kinase, $5 \mathrm{mM}$ phosphocreatine). Reaction mixtures were

661 incubated for $10 \mathrm{~min}$ at $4^{\circ} \mathrm{C}$ or $37^{\circ} \mathrm{C}$, as indicated. $5 \mu \mathrm{l}$ of reaction mixtures were used for analyses

662 by immunofluorescence microscopy. Fluorescence microscopy analyses were performed using

663 PEG silanized glass slides prepared as described (Alberti et al., 2018), and coverslips were

664 sealed with nail polish. DNA was stained with Hoechst 33258. Images were captured on an

665 inverted microscope using a 63x objective (NA 1.4 oil).

667 Immunofluorescence staining.

668 Cells grown on coverslips were fixed with PBS/4\% paraformaldehyde (PFA) for 15min at RT

669 followed by a 10min permeabilization step in PBS/0.2\% Triton X-100-PBS and blocked in

$670 \mathrm{PBS} / 3 \% \mathrm{BSA}$ for 30min. For immunofluorescence staining, primary antibodies and appropriate

671 secondary antibodies coupled to fluorochrome were diluted in blocking solution and incubated

672 for $1 \mathrm{~h}$ at RT. To detect endogenous TopBP1, cells were pre-treated with Cytoskeleton (CSK)

673 buffer before fixation. DNA was stained with Hoechst 33258 (Invitrogen, Cat H21491) and

674 coverslips were mounted on glass slides with Prolong Gold antifade reagent (Invitrogen, Cat

675 P36930). Images were captured using a 63x objective (NA 1.46 oil).

676

677 Fluorescence Recovery After Photo-bleaching (FRAP). 
678 For FRAP experiments, $U$-2-OS Tet-On cells were seeded into $\mu$-Dish ${ }^{35} \mathrm{~mm}$, high (Ibidi, 81156) and

679 incubated $24 \mathrm{~h}$ in the presence of $1 \mu \mathrm{g} / \mathrm{ml}$ doxycycline to induce expression of eGFP-TopBP1.

680 Imaging was realized using a 63x objective (NA 1.4). eGFP-TopBP1 bodies were photo-

681 bleached and the GFP signal intensity of 23 spots was measured before and during $5 \mathrm{~min}$

682 following photo-bleaching with an imaging frequency of 60 images/min.

683

\section{Opto-TopBP1 activation.}

685 Cells were plated at around $70 \%$ confluency in DMEM. Expression of opto-TopBP1 was

686 induced for $16 \mathrm{~h}$ with $2 \mu \mathrm{g} / \mathrm{ml}$ doxycycline. For light activation, plates were transferred into a

687 custom-made illumination box containing an array of 24 LEDs (488nm) delivering $10 \mathrm{~mW} / \mathrm{cm}^{2}$

688 (light intensity measured using a ThorLabs-PM16-121-power meter). Cry2 oligomerisation was

689 induced using $3 \mathrm{~min}$ of light-dark cycles (4s light followed by 10s dark). Images were captured

690 using a 63x objective (NA 1.46 oil). A Cell Profiler (version 2.2.0) pipeline was used to quantify

691 nuclear mCherry foci. Values were represented via violin plots or histograms elaborated in

692 Graph Pad Prism 8. Median and quartile values were indicated by continues and dashed lines

693 respectively. A non-parametric t-test (Mann-Withney) was used to compare mCherry spot/cell

694 distributions between samples. For each experiment, we performed three biological replicates

695 and we show one representative experiment.

\section{Live cell microscopy.}

698 Live imaging of opto-TopBP1 WT overexpressing cells was performed on an inverted 699 microscope (AxioObserver, Carl Zeiss, Germany) using a 63x oil immersion objective (NA 1.4).

700 Fluorescence was detected on a CMOS camera (ORCA-Flash4.0, Hamamatsu) with an exposure 
701 time set to $100 \mathrm{~ms}$ and at frame rate of 2 images $/ \mathrm{min}$. All recordings were carried out at $37^{\circ} \mathrm{C}$

702 under 5\% CO2. For Cry2 activation, cells were treated as explained before, under the

703 microscope, and imaged immediately after light activation.

705 Stimulated emission depletion (STED) super-resolution microscopy.

706 For Figure 2E, Flp-In ${ }^{T M} T-R E x^{T M} 293$ cells expressing opto-TopBP1 were exposed to blue light 707 (488nm) to induce TopBP1 foci, as described before. For STED microscopy, immunolabelling of 708 mCherry was realized using an anti-mCherry antibody followed by a secondary antibody coupled 709 to Atto-647N fluorochrome. Confocal and STED imaging was performed using a quad scanning

710 STED microscope (Expert Line, Abberior Instruments, Germany) equipped with a

711 PlanSuperApo 100x/1.40 oil immersion objective (Olympus, Japan). Atto-647N was excited at

$712640 \mathrm{~nm}$ with a dwell time of $10 \mu \mathrm{s}$ and STED was performed at $775 \mathrm{~nm}$. Images were collected in

713 line accumulation mode (5 lines accumulation). Fluorescence was detected using avalanche

714 photo diodes and spectral detection (650-750nm). The pinhole was set to 1.0 Airy units and a

715 pixel size of $10 \mathrm{~nm}$ was used for all acquisitions. A gating of 8ns was applied. Sphericity of opto-

716 TopBP1 condensates was assessed using IMARIS software (Bitplane). For dual color STED

717 imaging, Atto-647N and Abberior STAR Orange were used and respectively imaged at 640 and

$718561 \mathrm{~nm}$ excitation. Detection was set to 650-750nm for Atto-647N and 570-630nm for Abberior

719 STAR Orange. Other acquisition parameters were the same described as above.

\section{DNA fibers.}

722 Neo-synthesized DNA was sequentially pulse labelled with two halogenated thymidine analogs,

723 5-Iodo-2'-deoxyuridine (IdU at 25 $\mathrm{M}$ - Sigma \# I7125) and 5-Chloro-2'-deoxyuridine (CldU at 
$72450 \mu \mathrm{M}$ - Sigma \# C6891), for 20min. After IdU incorporation, cells were washed two times

725 before CldU addition. CldU incorporation (20min total) was conducted in the presence of $3 \mathrm{~min}$

726 of light-dark cycles (4s light followed by $10 \mathrm{~s}$ dark) each $7 \mathrm{~min}$, to assure opto-TopBP1

727 condensates formation and persistence. Cells were trypsinized and washed with ice cold PBS.

7282000 cells were drop on top of a microscope slide and let dry at least $5 \mathrm{~min}$ before lyse with $7 \mu \mathrm{L}$

729 of spreading buffer (200mM Tris.HCl pH7.5, 50mM EDTA, 0.5\% SDS) during 3min. DNA was

730 spread by tilting the slide and letting the drop running down slowly. Once air-dry, DNA spreads

731 were fixed in methanol/acetic acid 3:1 for $10 \mathrm{~min}$, denatured with $2.5 \mathrm{M} \mathrm{HCl}$ during $1 \mathrm{~h}$ and

732 blocked in PBS/1\% BSA/0.1\% Tween. DNA spreads were immunostained with mouse anti-

733 BrdU, rat anti-BrdU and mouse anti-ssDNA antibodies to detect IdU, CldU and intact DNA

734 fibers respectively. Corresponding secondary antibodies conjugated to Alexa Fluor dyes were

735 used in a second step. Images were captured using a 40x objective (NA 1.4 oil). The acquired

736 DNA fiber images were analyzed by using FIJI Software and statistical analysis of at least 150

737 IdU and CldU tracks on intact fibers was performed with GraphPad Prism 8. Mean values were

738 indicated by red lines. One-way ANOVA analysis was applied to compare means of samples in a

739 group. 


\section{References:}

Acevedo, J., Yan, S., and Michael, W.M. (2016). Direct Binding to Replication Protein A (RPA)-coated Single-stranded DNA Allows Recruitment of the ATR Activator TopBP1 to Sites of DNA Damage. J Biol Chem 291, 13124-13131.

Alberti, S., Saha, S., Woodruff, J.B., Franzmann, T.M., Wang, J., and Hyman, A.A. (2018). A User's Guide for Phase Separation Assays with Purified Proteins. J Mol Biol 430, 4806-4820.

Altmeyer, M., Neelsen, K.J., Teloni, F., Pozdnyakova, I., Pellegrino, S., Grofte, M., Rask, M.B., Streicher, W., Jungmichel, S., Nielsen, M.L., et al. (2015). Liquid demixing of intrinsically disordered proteins is seeded by poly(ADP-ribose). Nat Commun 6, 8088 .

Banani, S.F., Lee, H.O., Hyman, A.A., and Rosen, M.K. (2017). Biomolecular condensates: organizers of cellular biochemistry. Nat Rev Mol Cell Biol 18, 285-298.

Bass, T.E., and Cortez, D. (2019). Quantitative phosphoproteomics reveals mitotic function of the ATR activator ETAA1. J Cell Biol.

Bass, T.E., Luzwick, J.W., Kavanaugh, G., Carroll, C., Dungrawala, H., Glick, G.G., Feldkamp, M.D., Putney, R., Chazin, W.J., and Cortez, D. (2016). ETAA1 acts at stalled replication forks to maintain genome integrity. Nat Cell Biol 18, 1185-1195.

Boija, A., Klein, I.A., Sabari, B.R., Dall'Agnese, A., Coffey, E.L., Zamudio, A.V., Li, C.H., Shrinivas, K., Manteiga, J.C., Hannett, N.M., et al. (2018). Transcription Factors Activate Genes through the PhaseSeparation Capacity of Their Activation Domains. Cell.

Bonilla, C.Y., Melo, J.A., and Toczyski, D.P. (2008). Colocalization of sensors is sufficient to activate the DNA damage checkpoint in the absence of damage. Mol Cell 30, 267-276.

Bracha, D., Walls, M.T., and Brangwynne, C.P. (2019). Probing and engineering liquid-phase organelles. Nat Biotechnol 37, 1435-1445.

Bracha, D., Walls, M.T., Wei, M.T., Zhu, L., Kurian, M., Avalos, J.L., Toettcher, J.E., and Brangwynne, C.P. (2018). Mapping Local and Global Liquid Phase Behavior in Living Cells Using PhotoOligomerizable Seeds. Cell 175, 1467-1480 e1413.

Branon, T.C., Bosch, J.A., Sanchez, A.D., Udeshi, N.D., Svinkina, T., Carr, S.A., Feldman, J.L., Perrimon, N., and Ting, A.Y. (2018). Efficient proximity labeling in living cells and organisms with TurboID. Nat Biotechnol 36, 880-887.

Broderick, R., Nieminuszczy, J., Blackford, A.N., Winczura, A., and Niedzwiedz, W. (2015). TOPBP1 recruits TOP2A to ultra-fine anaphase bridges to aid in their resolution. Nat Commun 6,6572 .

Bugaj, L.J., Choksi, A.T., Mesuda, C.K., Kane, R.S., and Schaffer, D.V. (2013). Optogenetic protein clustering and signaling activation in mammalian cells. Nat Methods 10, 249-252.

Byun, T.S., Pacek, M., Yee, M.C., Walter, J.C., and Cimprich, K.A. (2005). Functional uncoupling of MCM helicase and DNA polymerase activities activates the ATR-dependent checkpoint. Genes Dev 19, 1040-1052.

Chowdhury, P., Lin, G.E., Liu, K., Song, Y., Lin, F.T., and Lin, W.C. (2014). Targeting TopBP1 at a convergent point of multiple oncogenic pathways for cancer therapy. Nat Commun 5, 5476.

Ciccia, A., and Elledge, S.J. (2010). The DNA damage response: making it safe to play with knives. Mol Cell 40, 179-204.

Delacroix, S., Wagner, J.M., Kobayashi, M., Yamamoto, K., and Karnitz, L.M. (2007). The Rad9-Hus1Rad1 (9-1-1) clamp activates checkpoint signaling via TopBP1. Genes Dev 21, 1472-1477.

Du, M., and Chen, Z.J. (2018). DNA-induced liquid phase condensation of cGAS activates innate immune signaling. Science 361, 704-709.

Duursma, A.M., Driscoll, R., Elias, J.E., and Cimprich, K.A. (2013). A role for the MRN complex in ATR activation via TOPBP1 recruitment. Mol Cell 50, 116-122.

Erdel, F., Rademacher, A., Vlijm, R., Tunnermann, J., Frank, L., Weinmann, R., Schweigert, E., Yserentant, K., Hummert, J., Bauer, C., et al. (2020). Mouse Heterochromatin Adopts Digital Compaction States without Showing Hallmarks of HP1-Driven Liquid-Liquid Phase Separation. Mol Cell 78, 236-249 e237. 
Franzmann, T.M., and Alberti, S. (2019). Protein Phase Separation as a Stress Survival Strategy. Cold Spring Harb Perspect Biol.

Franzmann, T.M., Jahnel, M., Pozniakovsky, A., Mahamid, J., Holehouse, A.S., Nuske, E., Richter, D., Baumeister, W., Grill, S.W., Pappu, R.V., et al. (2018). Phase separation of a yeast prion protein promotes cellular fitness. Science 359.

Friedberg, E.C., Walker, G.C., Siede, W., Wood, R.D., Schultz, R.A., and Elenberger, T. (2006). DNA repair and mutagenesis, 2nd edn (Washington, D.C.: ASM Press).

Garcia-Higuera, I., Taniguchi, T., Ganesan, S., Meyn, M.S., Timmers, C., Hejna, J., Grompe, M., and D'Andrea, A.D. (2001). Interaction of the Fanconi anemia proteins and BRCA1 in a common pathway. Mol Cell 7, 249-262. M., and Mailand, N. (2016). Activation of the ATR kinase by the RPA-binding protein ETAA1. Nat Cell Biol 18, 1196-1207.

804 Hashimoto, Y., and Takisawa, H. (2003). Xenopus Cut5 is essential for a CDK-dependent process in the initiation of DNA replication. EMBO J 22, 2526-2535.

806 Hashimoto, Y., Tsujimura, T., Sugino, A., and Takisawa, H. (2006). The phosphorylated C-terminal domain of Xenopus Cut5 directly mediates ATR-dependent activation of Chk1. Genes Cells 11, 9931007.

809 Hyman, A.A., and Simons, K. (2012). Cell biology. Beyond oil and water--phase transitions in cells. 810 Science 337, 1047-1049.

811 Jackson, S.P., and Bartek, J. (2009). The DNA-damage response in human biology and disease. Nature 812 461, 1071-1078.

813 Jackson, S.P., and Helleday, T. (2016). DNA REPAIR. Drugging DNA repair. Science 352, 1178-1179.

814 Kasahara, K., Goto, H., Enomoto, M., Tomono, Y., Kiyono, T., and Inagaki, M. (2010). 14-3-3gamma mediates Cdc25A proteolysis to block premature mitotic entry after DNA damage. EMBO J 29, 28022812.

Kilic, S., Lezaja, A., Gatti, M., Bianco, E., Michelena, J., Imhof, R., and Altmeyer, M. (2019). Phase separation of 53BP1 determines liquid-like behavior of DNA repair compartments. The EMBO Journal e101379.

Kumagai, A., Lee, J., Yoo, H.Y., and Dunphy, W.G. (2006). TopBP1 activates the ATR-ATRIP complex. Cell 124, 943-955.

Kwon, I., Kato, M., Xiang, S., Wu, L., Theodoropoulos, P., Mirzaei, H., Han, T., Xie, S., Corden, J.L., and McKnight, S.L. (2013). Phosphorylation-regulated binding of RNA polymerase II to fibrous polymers of low-complexity domains. Cell 155, 1049-1060.

Lecona, E., and Fernandez-Capetillo, O. (2018). Targeting ATR in cancer. Nat Rev Cancer 18, 586-595. Lee, Y.C., Zhou, Q., Chen, J., and Yuan, J. (2016). RPA-Binding Protein ETAA1 Is an ATR Activator Involved in DNA Replication Stress Response. Curr Biol 26, 3257-3268.

Leimbacher, P.A., Jones, S.E., Shorrocks, A.K., de Marco Zompit, M., Day, M., Blaauwendraad, J., Bundschuh, D., Bonham, S., Fischer, R., Fink, D., et al. (2019). MDC1 Interacts with TOPBP1 to Maintain Chromosomal Stability during Mitosis. Mol Cell.

Lin, S.J., Wardlaw, C.P., Morishita, T., Miyabe, I., Chahwan, C., Caspari, T., Schmidt, U., Carr, A.M., and Garcia, V. (2012). The Rad4(TopBP1) ATR-activation domain functions in G1/S phase in a chromatin-dependent manner. PLoS Genet 8, e1002801.

Lindahl, T. (1993). Instability and decay of the primary structure of DNA. Nature 362, 709-715.

Lindsey-Boltz, L.A., and Sancar, A. (2011). Tethering DNA damage checkpoint mediator proteins topoisomerase IIbeta-binding protein 1 (TopBP1) and Claspin to DNA activates ataxia-telangiectasia mutated and RAD3-related (ATR) phosphorylation of checkpoint kinase 1 (Chk1). J Biol Chem 286, 19229-19236.

839 Lisby, M., Rothstein, R., and Mortensen, U.H. (2001). Rad52 forms DNA repair and recombination 840 centers during S phase. Proc Natl Acad Sci U S A 98, 8276-8282. 
841

842

843

844

845

846

847

848

849

850

851

852

853

854

855

856

857

858

859

860

861

862

863

864

865

866

867

868

869

870

871

872

873

874

875

876

877

878

879

880

881

882

883

884

885

886

887

888

889

890

Liu, K., Bellam, N., Lin, H.Y., Wang, B., Stockard, C.R., Grizzle, W.E., and Lin, W.C. (2009). Regulation of p53 by TopBP1: a potential mechanism for p53 inactivation in cancer. Mol Cell Biol 29, 2673-2693.

Liu, S., Bekker-Jensen, S., Mailand, N., Lukas, C., Bartek, J., and Lukas, J. (2006). Claspin operates downstream of TopBP1 to direct ATR signaling towards Chk1 activation. Mol Cell Biol 26, 6056-6064.

Liu, Y., Cussiol, J.R., Dibitetto, D., Sims, J.R., Twayana, S., Weiss, R.S., Freire, R., Marini, F., Pellicioli, A., and Smolka, M.B. (2017). TOPBP1(Dpb11) plays a conserved role in homologous recombination DNA repair through the coordinated recruitment of 53BP1(Rad9). J Cell Biol 216, 623-639.

Lu, H., Yu, D., Hansen, A.S., Ganguly, S., Liu, R., Heckert, A., Darzacq, X., and Zhou, Q. (2018). Phaseseparation mechanism for C-terminal hyperphosphorylation of RNA polymerase II. Nature 558, 318-323.

Makiniemi, M., Hillukkala, T., Tuusa, J., Reini, K., Vaara, M., Huang, D., Pospiech, H., Majuri, I., Westerling, T., Makela, T.P., et al. (2001). BRCT domain-containing protein TopBP1 functions in DNA replication and damage response. J Biol Chem 276, 30399-30406.

Mao, Y.S., Sunwoo, H., Zhang, B., and Spector, D.L. (2011). Direct visualization of the cotranscriptional assembly of a nuclear body by noncoding RNAs. Nat Cell Biol 13, 95-101.

Marechal, A., and Zou, L. (2013). DNA damage sensing by the ATM and ATR kinases. Cold Spring Harb Perspect Biol 5.

Maser, R.S., Monsen, K.J., Nelms, B.E., and Petrini, J.H. (1997). hMre11 and hRad50 nuclear foci are induced during the normal cellular response to DNA double-strand breaks. Mol Cell Biol 17, 6087-6096.

McSwiggen, D.T., Hansen, A.S., Teves, S.S., Marie-Nelly, H., Hao, Y., Heckert, A.B., Umemoto, K.K., Dugast-Darzacq, C., Tjian, R., and Darzacq, X. (2019). Evidence for DNA-mediated nuclear compartmentalization distinct from phase separation. Elife 8.

Michelena, J., Gatti, M., Teloni, F., Imhof, R., and Altmeyer, M. (2019). Basal CHK1 activity safeguards its stability to maintain intrinsic S-phase checkpoint functions. J Cell Biol 218, 2865-2875.

Mordes, D.A., Glick, G.G., Zhao, R., and Cortez, D. (2008). TopBP1 activates ATR through ATRIP and a PIKK regulatory domain. Genes Dev 22, 1478-1489.

Moudry, P., Watanabe, K., Wolanin, K.M., Bartkova, J., Wassing, I.E., Watanabe, S., Strauss, R., Troelsgaard Pedersen, R., Oestergaard, V.H., Lisby, M., et al. (2016). TOPBP1 regulates RAD51 phosphorylation and chromatin loading and determines PARP inhibitor sensitivity. J Cell Biol 212, 281288.

Munk, S., Sigurethsson, J.O., Xiao, Z., Batth, T.S., Franciosa, G., von Stechow, L., Lopez-Contreras, A.J., Vertegaal, A.C.O., and Olsen, J.V. (2017). Proteomics Reveals Global Regulation of Protein SUMOylation by ATM and ATR Kinases during Replication Stress. Cell Rep 21, 546-558.

Ochs, F., Karemore, G., Miron, E., Brown, J., Sedlackova, H., Rask, M.B., Lampe, M., Buckle, V., Schermelleh, L., Lukas, J., et al. (2019). Stabilization of chromatin topology safeguards genome integrity. Nature 574, 571-574.

Park, M.S., Knauf, J.A., Pendergrass, S.H., Coulon, C.H., Strniste, G.F., Marrone, B.L., and MacInnes, M.A. (1996). Ultraviolet-induced movement of the human DNA repair protein, Xeroderma pigmentosum type G, in the nucleus. Proc Natl Acad Sci U S A 93, 8368-8373.

Patel, A., Lee, H.O., Jawerth, L., Maharana, S., Jahnel, M., Hein, M.Y., Stoynov, S., Mahamid, J., Saha, S., Franzmann, T.M., et al. (2015). A Liquid-to-Solid Phase Transition of the ALS Protein FUS Accelerated by Disease Mutation. Cell 162, 1066-1077.

Pessina, F., Giavazzi, F., Yin, Y., Gioia, U., Vitelli, V., Galbiati, A., Barozzi, S., Garre, M., Oldani, A., Flaus, A., et al. (2019). Functional transcription promoters at DNA double-strand breaks mediate RNAdriven phase separation of damage-response factors. Nat Cell Biol.

Prior, I.A., Muncke, C., Parton, R.G., and Hancock, J.F. (2003). Direct visualization of Ras proteins in spatially distinct cell surface microdomains. J Cell Biol 160, 165-170.

Riback, J.A., Katanski, C.D., Kear-Scott, J.L., Pilipenko, E.V., Rojek, A.E., Sosnick, T.R., and Drummond, D.A. (2017). Stress-Triggered Phase Separation Is an Adaptive, Evolutionarily Tuned Response. Cell 168, 1028-1040 e1019. 
891

892

893

894

895

896

897

898

899

900

901

902

903

904

905

906

907

908

909

910

911

912

913

914

915

916

917

918

919

920

921

922

923

924

925

926

927

928

929

930

931

932

933

934

935

936

937

938

939

Sabari, B.R., Dall'Agnese, A., Boija, A., Klein, I.A., Coffey, E.L., Shrinivas, K., Abraham, B.J., Hannett, N.M., Zamudio, A.V., Manteiga, J.C., et al. (2018). Coactivator condensation at super-enhancers links phase separation and gene control. Science 361 .

Saldivar, J.C., Cortez, D., and Cimprich, K.A. (2017). The essential kinase ATR: ensuring faithful duplication of a challenging genome. Nat Rev Mol Cell Biol 18, 622-636.

Shin, Y., Berry, J., Pannucci, N., Haataja, M.P., Toettcher, J.E., and Brangwynne, C.P. (2017). Spatiotemporal Control of Intracellular Phase Transitions Using Light-Activated optoDroplets. Cell 168, $159-171$ e114.

Shin, Y., and Brangwynne, C.P. (2017). Liquid phase condensation in cell physiology and disease. Science 357.

Shin, Y., Chang, Y.C., Lee, D.S.W., Berry, J., Sanders, D.W., Ronceray, P., Wingreen, N.S., Haataja, M., and Brangwynne, C.P. (2018). Liquid Nuclear Condensates Mechanically Sense and Restructure the Genome. Cell 175, 1481-1491 e1413.

Soding, J., Zwicker, D., Sohrabi-Jahromi, S., Boehning, M., and Kirschbaum, J. (2020). Mechanisms for Active Regulation of Biomolecular Condensates. Trends Cell Biol 30, 4-14.

Sokka, M., Rilla, K., Miinalainen, I., Pospiech, H., and Syvaoja, J.E. (2015). High levels of TopBP1 induce ATR-dependent shut-down of rRNA transcription and nucleolar segregation. Nucleic Acids Res 43, 4975-4989.

Thada, V., and Cortez, D. (2019). Common motifs in ETAA1 and TOPBP1 required for ATR kinase activation. J Biol Chem 294, 8395-8402.

Tian, T., Harding, A., Inder, K., Plowman, S., Parton, R.G., and Hancock, J.F. (2007). Plasma membrane nanoswitches generate high-fidelity Ras signal transduction. Nat Cell Biol 9, 905-914.

Tubbs, A., and Nussenzweig, A. (2017). Endogenous DNA Damage as a Source of Genomic Instability in Cancer. Cell 168, 644-656.

Van, C., Yan, S., Michael, W.M., Waga, S., and Cimprich, K.A. (2010). Continued primer synthesis at stalled replication forks contributes to checkpoint activation. J Cell Biol 189, 233-246.

Vidal-Eychenie, S., Decaillet, C., Basbous, J., and Constantinou, A. (2013). DNA structure-specific priming of ATR activation by DNA-PKcs. J Cell Biol 202, 421-429.

Wang, X., Ran, T., Zhang, X., Xin, J., Zhang, Z., Wu, T., Wang, W., and Cai, G. (2017). 3.9 A structure of the yeast Mec1-Ddc2 complex, a homolog of human ATR-ATRIP. Science 358, 1206-1209.

Woodruff, J.B., Ferreira Gomes, B., Widlund, P.O., Mahamid, J., Honigmann, A., and Hyman, A.A. (2017). The Centrosome Is a Selective Condensate that Nucleates Microtubules by Concentrating Tubulin. Cell 169, 1066-1077 e1010.

Wright, R.H., Dornan, E.S., Donaldson, M.M., and Morgan, I.M. (2006). TopBP1 contains a transcriptional activation domain suppressed by two adjacent BRCT domains. Biochem J 400, 573-582.

Yan, S., and Michael, W.M. (2009). TopBP1 and DNA polymerase alpha-mediated recruitment of the 91-1 complex to stalled replication forks: implications for a replication restart-based mechanism for ATR checkpoint activation. Cell Cycle 8, 2877-2884.

Yoo, H.Y., Kumagai, A., Shevchenko, A., and Dunphy, W.G. (2009). The Mre11-Rad50-Nbs1 complex mediates activation of TopBP1 by ATM. Mol Biol Cell 20, 2351-2360.

Zhang, P., Fan, B., Yang, P., Temirov, J., Messing, J., Kim, H.J., and Taylor, J.P. (2019). Chronic optogenetic induction of stress granules is cytotoxic and reveals the evolution of ALS-FTD pathology. Elife 8.

Zhou, Z.W., Liu, C., Li, T.L., Bruhn, C., Krueger, A., Min, W., Wang, Z.Q., and Carr, A.M. (2013). An essential function for the ATR-activation-domain (AAD) of TopBP1 in mouse development and cellular senescence. PLoS Genet 9, e1003702.

Zou, L., and Elledge, S.J. (2003). Sensing DNA damage through ATRIP recognition of RPA-ssDNA complexes. Science 300, 1542-1548. 
bioRxiv preprint doi: https://doi.org/10.1101/2020.10.05.326181; this version posted October 5, 2020. The copyright holder for this preprint (which was not certified by peer review) is the author/funder, who has granted bioRxiv a license to display the preprint in perpetuity. It is made available under aCC-BY-NC-ND 4.0 International license.

940 


\section{$941 \quad$ Figure Legends}

942 Figure 1. Optogenic control of TopBP1 biomolecular condensates. A) Schematic

943 representation of the opto-TopBP1 construct. Full length WT or W1145R TopBP1 is fused at the

944 N-terminus to TurboID, an optimized biotin ligase that can modify proteins within minutes, and

945 at the C-terminus with mCherry fluorescent protein and the Cryptochrome 2 (Cry2) protein.

946 Upon exposure to 488nm light, Cry2 oligomerises and induces TopBP1 condensation into

947 mCherry positive foci. B) Representative fluorescence images of cells expressing WT and

948 W1145R opto-TopBP1, before (Light OFF) and after (Light ON) 3min exposure to cycles of 4s

949 light (488nm)-10s resting. Control cells express the opto-module mCherry-Cry2. C-D)

950 Schematic representation of the opto-TopBP1 chimeric protein opto-TopBP1 ${ }^{\text {ETAA1-AAD }}(\mathbf{C})$ and

951 opto-AAD-NLS ${ }^{\text {TopBP1 }}$ (D). TopBP1 ATR Activation Domain (AAD) was replaced by ETAA1

952 AAD $(\mathbf{C})$ or cloned into the opto-module together with the TopBP1 Nuclear Localisation

953 Sequence (NLS) (D). Representative fluorescence images of opto-TopBP1 ${ }^{\text {ETAA1-AAD }}(\mathbf{C})$ and

954 opto-AAD-NLS ${ }^{\text {TopBP1 }}$ (D) exposed (Light ON) or not (Light OFF) to cycles of 4s light (488nm)-

955 10s resting for $3 \mathrm{~min}$. Western blotting with the indicated antibodies showing opto-TopBP1, opto-

956 TopBP1 $^{\text {ETAA1-AAD }}(\mathbf{C})$ and opto-AAD-NLS ${ }^{\text {TopBP1 }}(\mathbf{D})$ expression. FL in D stands for Full Length

957 and corresponds to opto-TopBP1 construct. (B-C-D) DNA was stained with Hoechst 33258.

958 Scale bars: $10 \mu \mathrm{m}$. Violin plot represents distribution of number of mCherry foci per cell. Median

959 and quartile values are represented by continues and dashed lines respectively. The statistical

960 significance of the difference in mCherry spot/cell distributions between samples is represented

961 by $*$.

962 
963 Figure 2. Properties of TopBP1 optogenetic and endogenous condensates. A) 3D analysis of

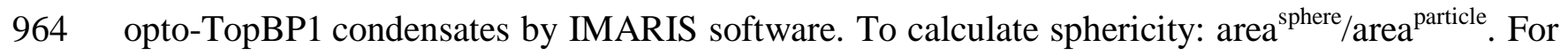

965 a sphere the value is 1 . We represented the 3D projection and the spherical value for particle 1

966 (P1). Other particles were analyzed for their spherical values (P2=0.932587; P3=0.918362;

967 P4=0.949144; P5=0.935103). B) Fluorescence images representing a fusion event in cells

968 expressing opto-TopBP1 WT activated by light (3min exposure to cycles of 4s light-10s resting).

969 C) Histograms representing the mean of mCherry foci per cell. Cells expressing opto-TopBP1

970 WT were first exposed for 3 min to cycles of 4s light (488nm)-10s resting (Light ON) and then

971 left into dark from 5 to $25 \mathrm{~min}$. D) Violin plot representing the number of mCherry foci per cell

972 in cells expressing opto-TopBP1 WT after (Light ON) 3min exposure to cycles of 4s light

973 (488nm)-10s resting. When indicated, cells were pre-treated with $0.5 \mathrm{M}$ sucrose or $0.4 \mathrm{M}$ sorbitol

974 for 1h. E) Violin plot representing the number of endogenousTopBP1 foci per cell in cells

975 treated with $5 \mathrm{mM} \mathrm{HU}$ for $2 \mathrm{~h}$. When indicated, cells were simultaneously incubated with $0.5 \mathrm{M}$

976 sucrose or $0.4 \mathrm{M}$ sorbitol for $1 \mathrm{~h}$. Represented values correspond to the sum of three independent

977 experiments. D-E) Median and quartile values are represented by continues and dashed lines

978 respectively. The statistical significance of the difference in mCherry or TopBP1 foci/cell

979 distributions between samples is represented by *. F-G) Super-resolution STED of opto-TopBP1

980 foci $(\mathbf{F})$ and endogenous TopBP1 (G) foci identified with anti-mCherry (F-left panel) or anti-

981 TopBP1 (F-right panel and G) antibodies. Opto-TopBP1 foci were induced by light (F), while

982 endogenous TopBP1 foci by $5 \mathrm{mM}$ HU for $2 \mathrm{~h}(\mathbf{G})$. Scale bars: $2 \mu \mathrm{m}$ for CONFOCAL images and

$9831 \mu \mathrm{m}$ for STED images. 
985 Figure 3. TopBP1 ${ }^{\text {b6-8 }}$ phase separates in vitro. A) Schematic representation of recombinant

986 WT and W1145R TopBP1 (TopBP1 ${ }^{\text {b6-8 }}$ ) expressed in insect cells. The protein includes TopBP1

987 amino acids 884 to 1522 , which comprise BRCT6-AAD-BRCT7-8. It is tagged with 6xHistidine

988 and maltose binding protein (MBP) at its N-terminus, and monomeric Green Fluorescent Protein

989 (GFP) at the C-terminus. PreScission (3C) and TEV protease sites are indicated. B)

990 Representative images of TopBP1 droplets formed using $10 \mu \mathrm{M}$ of WT or W1145R TopBP1 ${ }^{\mathrm{b} 6-8}-$

991 GFP incubated in phosphate buffer $\mathrm{pH} 7.6$ containing $150 \mathrm{mM}$ KAc and, when indicated, $2 \%$

992 PEG. Scale bars: $10 \mu \mathrm{m}$. C-D) Representative images of TopBP1 $1^{\text {b6-8 }}$-RFP $(1 \mu \mathrm{M})(\mathbf{C})$ and double-

993 stranded DNA $(300 \mathrm{nM})$ (D) trapping by pre-formed TopBP1 $1^{\mathrm{b} 6-8}$-GFP $(10 \mu \mathrm{M})$ droplets in

994 phosphate buffer $\mathrm{pH}$ 7.6, 150mM KAc, 2\% PEG. The design of the experiment is represented

995 schematically. DNA was stained with Hoechst 33258 (D). Scale bars: $10 \mu \mathrm{m}$. Line scan of GFP-

996 RFP (C) and GFP-Hoechst (D) signals is used to analyze co-localisation. E) Confocal image of

997 purified $(2.5 \mu \mathrm{M})$ WT TopBP1 ${ }^{\text {b6-8 }}$ in the presence of 40ng $2.9 \mathrm{~kb}$ circular DNA plasmid DNA

998 marked with Hoechst 33258. Scale bar: $2 \mu \mathrm{m}$. F) Purified $(2 \mu \mathrm{M})$ WT and W1145R TopBP1 ${ }^{\text {b6-8 }}$

999 were incubated for $10 \mathrm{~min}$ at $37^{\circ} \mathrm{C}$ with "Dignam and Roeder" nuclear extracts $(0.2 \mu \mathrm{g} / \mu \mathrm{l})$, in

1000 physiological conditions (as described in Methods), and then analyzed by fluorescence

1001 microscopy for condensates in suspension. Control images of TopBP1 ${ }^{\text {b6-8 }}$-GFP $(10 \mu \mathrm{M})$ alone

1002 and of protein extract $(0.2 \mu \mathrm{g} / \mu \mathrm{l})$ are shown. Scale bars: $10 \mu \mathrm{m}$.

1003

1004 Figure 4. Reconstitution of TopBP1 protein network by BioID coupled to MS. A) Schematic

1005 representation of Flag-BirA*-TopBP1. In the presence of biotin (green dots), the biotin ligase

1006 BirA* biotinylates proteins within a 10nm range. B-C) Proteins identified by proximity-

1007 dependent biotin labelling in cells expressing WT or W1145R Flag-BirA*_TopBP1 were ranked 
1008 according to their iBAQ value $(\mathbf{B})$ or compared in a Volcano plot $(\mathbf{C})$. Cells were grown for 16hr

1009 in presence of $1 \mu \mathrm{g} / \mathrm{ml}$ doxycycline and incubated for $3 \mathrm{~h}$ with $50 \mu \mathrm{M}$ biotin. For Volcano plot $(\mathbf{C})$,

1010 a standard t-test was used to evaluate differences in protein abundance between samples. The left

1011 and right parts of volcano plot represent proteins enriched in W1145R and WT sample,

1012 respectively. Proteins depicted in the center part of the plot are common to both samples. D)

1013 Streptavidin pulldowns of proteins biotinylated by WT and W1145R Flag-BirA*-TopBP1 were

1014 probed for the indicated proteins by immunoblotting. When indicated (+), cells were treated with

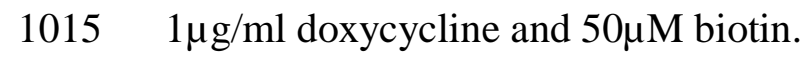

1016

1017 Figure 5. Functional consequences of TopBP1 condensation. A) Western blot analysis of

1018 TopBP1 and Chk1/phospho Chk1 (Ser345) of cells expressing WT and W1145R opto-TopBP1,

1019 before (Light OFF) and after (Light ON) 3min exposure to cycles of 4s light (488nm)-10s

1020 resting. Control cells express the opto-module mCherry-Cry2. B) Western blot analysis of

1021 TopBP1/pTopBP1 (Ser1138) and Chk1/pChk1 (Ser345) of cells expressing WT opto-TopBP1

1022 during reiterative cycles of optogenetic activation (3min exposure to cycles of $4 \mathrm{~s}$ light $-10 \mathrm{~s}$

1023 resting). 4 cycles each $20 \mathrm{~min}$. Total time: $92 \mathrm{~min}$. C) Streptavidin pulldowns of proteins

1024 biotinylated by optogenetic activated WT and W1145R TurboID-TopBP1 were probed for the

1025 indicated proteins by immunoblotting. When indicated $(+)$, cells were simultaneously incubated

1026 with $500 \mu \mathrm{M}$ of biotin and exposed to blue light for 10min of light-dark cycles (4s light followed

1027 by 30 s dark). D) Super-resolution STED of opto-TopBP1 and pATR (Thr1989) foci. Co-

1028 localisation between opto-TopBP1 (anti-mCherry) and pATR is indicated by line scan. Scale bar:

$10291 \mu \mathrm{m}$. E) CldU/IdU incorporation ratio of cells expressing WT and W1145R opto-TopBP1, 1030 before (-) and after (+) 3min exposure to cycles of 4s light (488nm)-10s resting. Mean values are 
1031 represented by red lines and the statistical significance among them is represented by *. Control

1032 cells express the opto-module.

1033

1034 Figure 6. Regulation of TopBP1 condensation. A) (Left panel) Violin plot representing the 1035 number of mCherry foci per cell in cells expressing opto-TopBP1 WT after (Light ON) 3min 1036 exposure to cycles of 4s light (488nm)-10s resting. When indicated, cells were pre-treated with $103710 \mu \mathrm{M}$ ATR inhibitors ETP-46464 or VE-821 for 1h. Median and quartile values are represented 1038 by continues and dashed lines respectively. (Right panel) Cells used for optogenetic experiments 1039 were probed for the indicated proteins by immunoblotting. The statistical significance of the 1040 difference in mCherry or TopBP1 foci/cell distributions between samples is represented by *. B) 1041 Alignment of ATR Activation Domain (AAD) sequences from Human (reference), Mouse and 1042 Xenopus. Amino acids from 1056 to 1155 of human TopBP1 are represented. Stars (*) on top of 1043 the alignment represent amino acids conserved among the three species. Conserved amino acids 1044 in mTopBP1 and xTopBP1 sequences are represented by single dots (.). Highly conserved amino 1045 acids F1071, S1138 and W1145 are indicated. C) (Left panel) Violin plot representing the 1046 number of mCherry foci per cell in cells expressing WT, F1071A, S1138A and S1138D opto1047 TopBP1 after (Light ON) 3min exposure to cycles of 4s light (488nm)-10s resting. Median and 1048 quartile values are represented by continues and dashed lines respectively. The statistical 1049 significance of the difference in mCherry or TopBP1 foci/cell distributions between WT and 1050 mutants samples is represented by *. (Right panel) Cells used for optogenetic experiments were 1051 probed for the indicated proteins by immunoblotting. D) Streptavidin pulldowns of proteins 1052 biotinylated by optogenetic activated WT, F1071A, S1138A and S1138D TurboID-TopBP1 were 1053 probed for the indicated proteins by immunoblotting. When indicated (+), cells were 
1054 simultaneously incubated with $500 \mu \mathrm{M}$ of biotin and exposed to blue light for 10min of light-dark

1055 cycles (4s light followed by 30 s dark).

1056

1057 Figure 7. TopBP1 condensation switches on ATR/Chk1 signalling. Model showing the 1058 accumulation of ATR/ATRIP and TopBP1 on RPA coated single-stranded DNA. Interaction 1059 with ATR through the ATR Activation Domain (AAD), phosphorylation of TopBP1 and ATR 1060 kinase activity induce TopBP1 condensation. The enrichment of molecular reactants in TopBP1 1061 compartments stimulates ATR activity above a threshold required for Chk1 activation and signal 1062 amplification.

1063

1064 Figure S1. Relates to Figure 1. A) Cells used for optogenetic experiments in Fig 1B were 1065 analyzed by western blotting using the indicated antibodies. B) (Left) Representative 1066 fluorescence images of cells expressing (+Dox) or not (-Dox) opto-RAD9 construct before 1067 (Light OFF) and after (Light ON) 3min exposure to cycles of 4s light (488nm)-10s resting. 1068 (Right) Western blotting from cells used for optogenetic experiments. C) Schematic 1069 representation of the opto-ETAA1 with representative fluorescence images of cells expressing 1070 the respective optogenetic construct before (Light OFF) and after (Light ON) 3min exposure to 1071 cycles of 4s light (488nm)-10s resting. Western blotting of the indicated proteins is represented.

1072 Violin plot represents the number of mCherry foci per cell. Median and quartile values are 1073 represented by continues and dashed lines respectively. The statistical significance of the 1074 difference in mCherry spot/cell distributions between samples is represented by *. B-C) DNA 1075 stained with Hoechst 33258. Scale bars: $10 \mu \mathrm{m}$. 
1077 Figure S2. Relates to Figure 2. A) Representative fluorescence images of cells used for

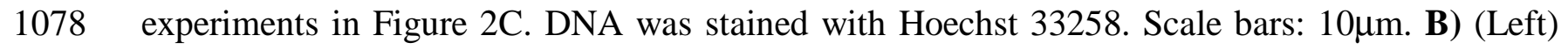

1079 Fluorescence Recovery After Photobleaching (FRAP) analyses of eGFP-TopBP1 bodies

1080 expressed in $U-2-O S$ cells. FRAP recordings of two individual eGFP-TopBP1 bodies are

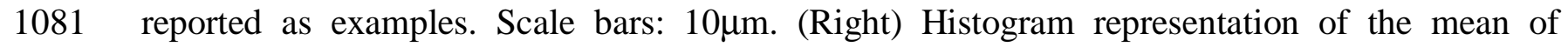

1082 immobile and mobile fractions per nucleus (13 nuclei, 23 individual spots). C) Representative

1083 fluorescence images of eGFP-TopBP1 expressing $U-2-O S$ cells treated, when indicated, with 3\%

1084 of 1,6 Hexanediol for 5min. D-E) Representative fluorescence images of cells used for

1085 experiments in Figure 2D (D) and 2E (E). DNA was stained with Hoechst 33258. Scale bars:

$108610 \mu \mathrm{m}$. F) Violin plot representing the number of mCherry foci per cell in cells expressing opto-

1087 TopBP1 WT treated with $5 \mathrm{mM}$ HU for $2 \mathrm{~h}$ and not exposed to blue light. TopBP1 foci were

1088 identified with anti-mCherry antibody after CSK treatment. Median and quartile values are

1089 represented by continues and dashed lines respectively. The statistical significance of the

1090 difference in mCherry foci/cell distributions between samples is represented by *.

1092 Figure S3. Relates to Figure 3. A) Representative fluorescence images of cells expressing opto-

1093 TopBP1 ${ }^{\text {b6-8 }}$ WT before (Light OFF) and after (Light ON) 3 min exposure to cycles of $4 \mathrm{~s}$ light

1094 (488nm)-10s resting. Control cells express the opto-module\# mCherry-Cry2. DNA was stained

1095 with Hoechst 33258. Western blotting of the indicated proteins is represented. Violin plot

1096 represents the number of mCherry foci per cell. Median and quartile values are represented by

1097 continues and dashed lines respectively. The statistical significance of the difference in mCherry

1098 spot/cell distributions between samples is represented by *. (B) Representative fluorescence

1099 microscopy images obtained with increasing concentrations of TopBP1 in the presence of 
1100 phosphate buffer $\mathrm{pH} 7.6$ containing 150mM KAc and 2\% PEG. (C) Representative fluorescent

1101 images of Figure 3F with DNA and merge panels. Images of condensates in suspension are

1102 represented. DNA was stained with Hoechst 33258. Line scan of GFP-Hoechst signals is used to

1103 analyze co-localisation. (A-B-C) Scale bars: $10 \mu \mathrm{m}$.

1105 Figure S4. Relates to Figure 4. Immunofluorescence staining of Flag-BirA*-TopBP1 WT using 1106 anti-Flag antibody. Biotin conjugates were revealed using AlexaFluor streptavidin. When 1107 indicated, cells were grown in the presence of $1 \mu \mathrm{g} / \mathrm{ml}$ doxycycline and $50 \mu \mathrm{M}$ biotin. DNA was 1108 stained with Hoechst 33258. Scale bars: $10 \mu \mathrm{m}$.

Figure S5. Relates to Figure 5. A) Western blot analysis of Chk1/phospho Chk1 (Ser345) in

1111 reaction mixtures containing increasing concentration of "Dignam and Roeder" nuclear extracts,

1112 and, when indicated, the ATR inhibitor ETP-46464 (10 $\mu \mathrm{M})$. Reaction mixtures were incubated

1113 for $10 \mathrm{~min}$ at $4^{\circ} \mathrm{C}$ or $37^{\circ} \mathrm{C}$, as indicated, in the presence of $150 \mathrm{mM}$ KAc in phosphate buffer $\mathrm{pH}$

1114 7.6. * represents unspecific bands. The design of the experiment is represented schematically. B)

1115 Western blot analysis of Chk1/pChk1 (Ser345) in reaction mixtures containing $4 \mu \mathrm{g} / \mu \mathrm{l}$ of

1116 "Dignam and Roeder" nuclear extracts in phosphate buffer $\mathrm{pH} 7.6$ supplemented with 150mM

1117 KAc. Reaction mixtures were incubated for $10 \mathrm{~min}$ at $4^{\circ} \mathrm{C}$ or $37^{\circ} \mathrm{C}$ and pre-incubation of extracts

1118 with ethidium bromide (EtBr) is indicated. C) Western blot analysis of TopBP1 and Chk1/pChk1

1119 (Ser345-Ser296) of cells expressing opto-TopBP1, exposed to 3 minutes cycles of 4s light

1120 (488nm)-10s resting. When indicated, cells were pre-treated with $10 \mu \mathrm{M}$ Chk1 inhibitor UCN-01.

1121 D-E-F) Immunofluorescence staining of opto-TopBP1 expressing cells activated by light and 1122 RAD9 (D) pATR (Thr1989) (E) and pChk1 (Ser345) (F) using specific antibodies. Line scans 
1123 are used to analyze co-localisation. DNA was stained with Hoechst 33258. Scale bars: $10 \mu \mathrm{m}$.

1124 Violin plot (F-right) representing spot of pChk1 in cells with (dashed white line) or without

1125 (solid white line) opto-TopBP1 foci. G) Histograms representing the mean of mCherry foci per

1126 cell. Cells expressing opto-TopBP1 WT were first exposed for $3 \mathrm{~min}$ to cycles of $4 \mathrm{~s}$ light

1127 (488nm)-10s resting (Light ON) and then treated (+) or not (-) with Cytoskeleton (CSK) buffer

1128 to keep chromatin-bound proteins.

1129

1130 Figure S6. Relates to Figure 6. A) Representative fluorescence images of cells used for

1131 optogenetic experiment in Figure 6A. B) Representative fluorescence images of cells used for 1132 optogenetic experiment in Figure 6C. 
A

opto-TopBP1

AAD

Blue Light

$(488 \mathrm{~nm})$

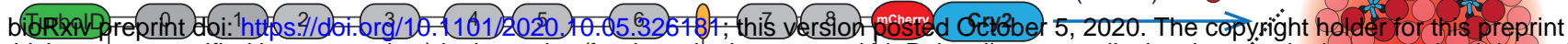
(which was not certified by peer review) is the author/funder, who has granted bioRxiv a license to display the preprint in perpetuity. It is made

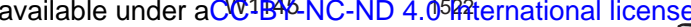

B
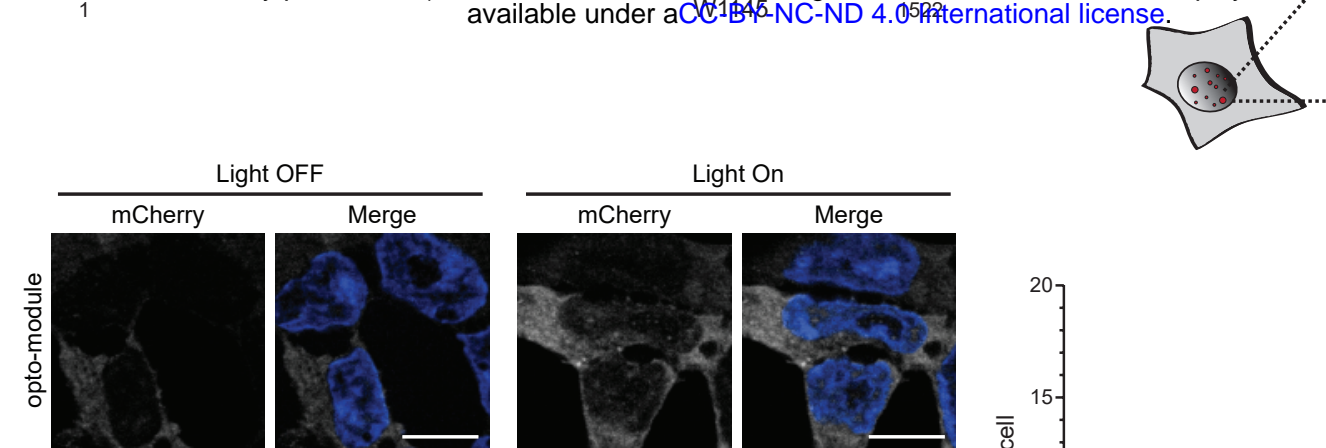

Light On

TopBP1 optodroplet
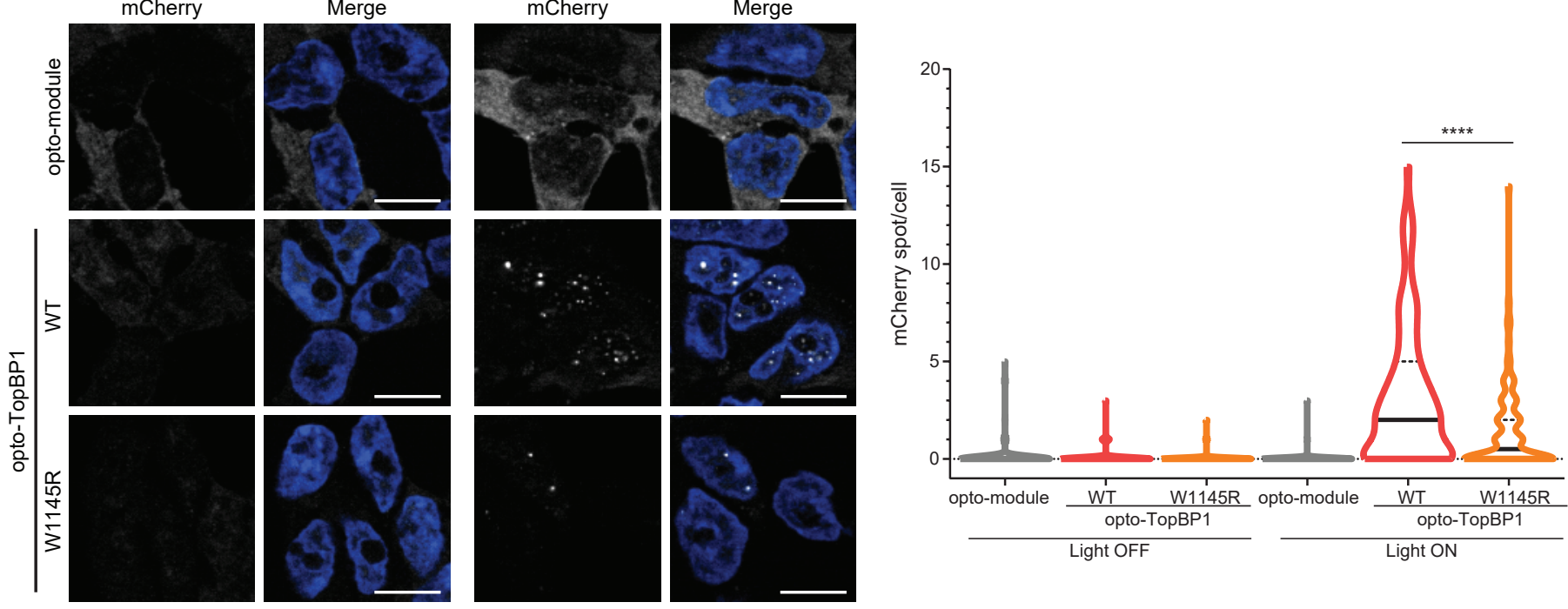

C opto-TopBP1 $^{\text {ETAA1-AAD }}$
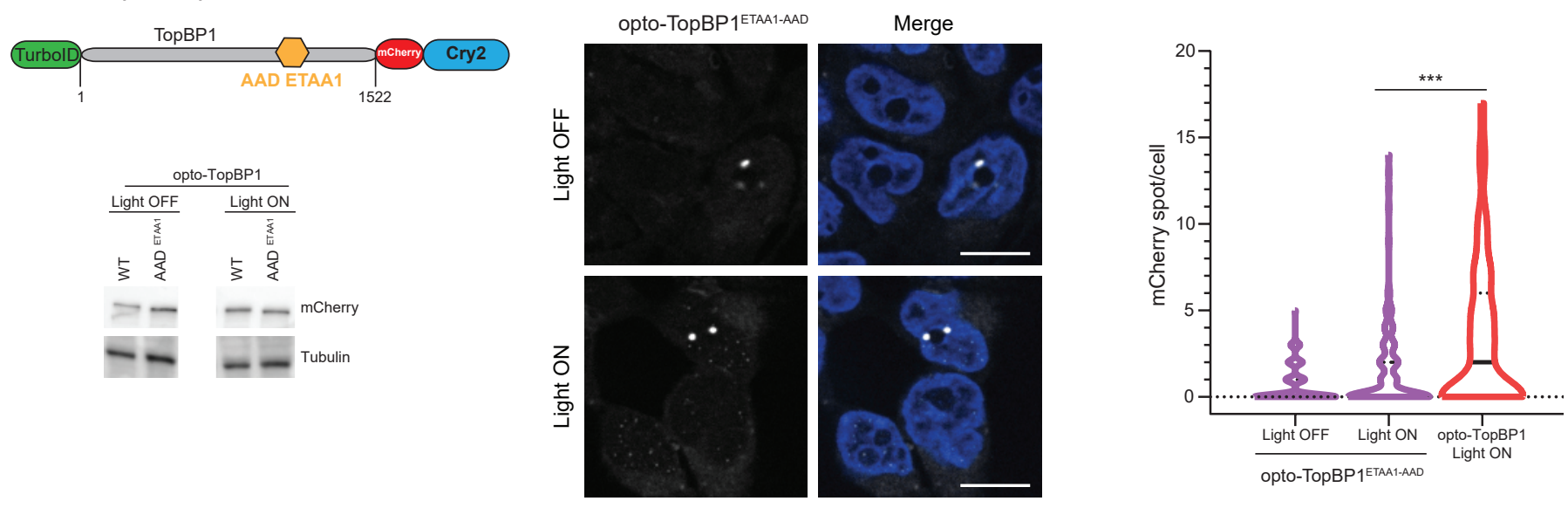

D opto-AAD-NLSTopBP1
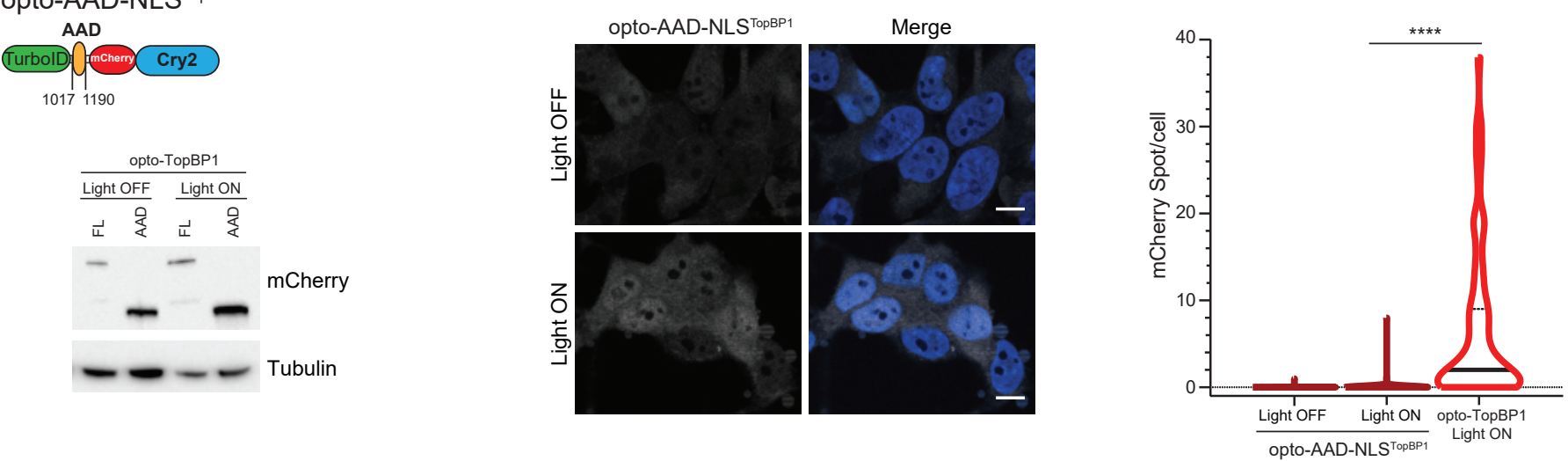

Figure 1 
A

sphere
bioRxiv preprint doi: https://doi.org/10.110 (which was rot certified by peer review) is th

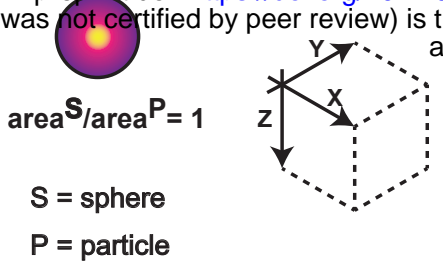

B

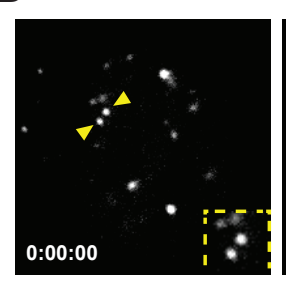

C

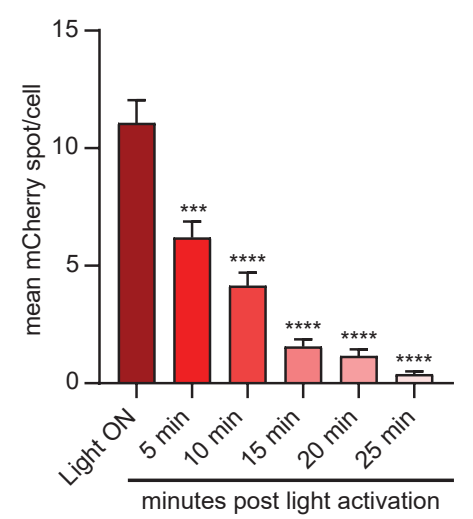

F anti-mCherry

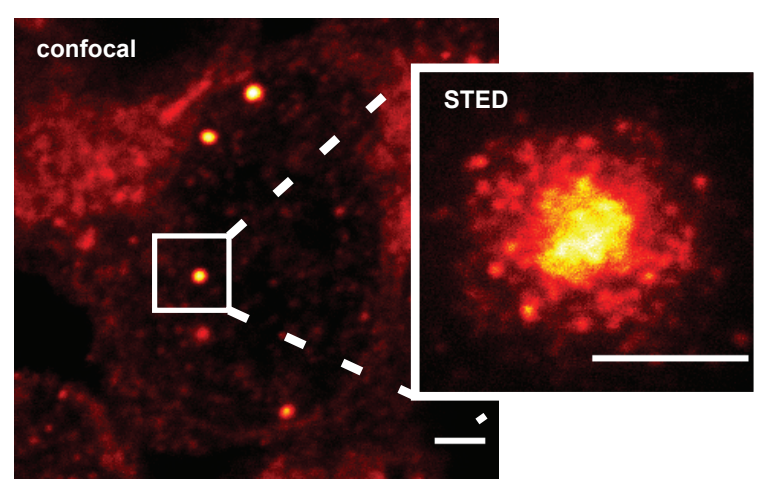

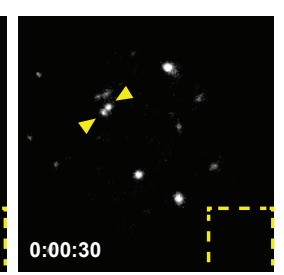

$0: 01: 00$
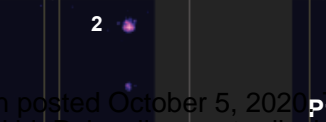

P1

a.

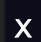

Z copyright holder for this preprint he preprint in perpetuity. It is made

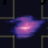

$$
\operatorname{area} \mathrm{S}_{\text {area }} \mathrm{P}^{1}=0.940223
$$
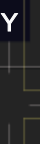

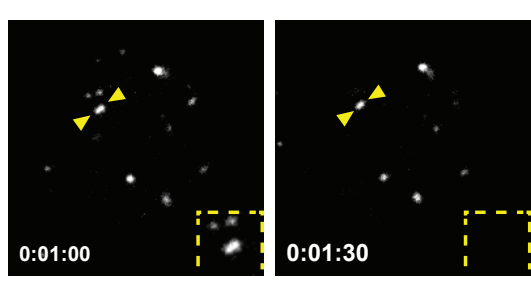

D

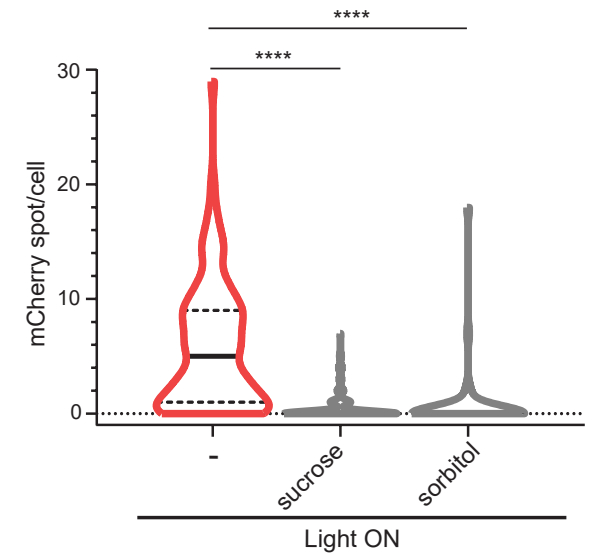

E
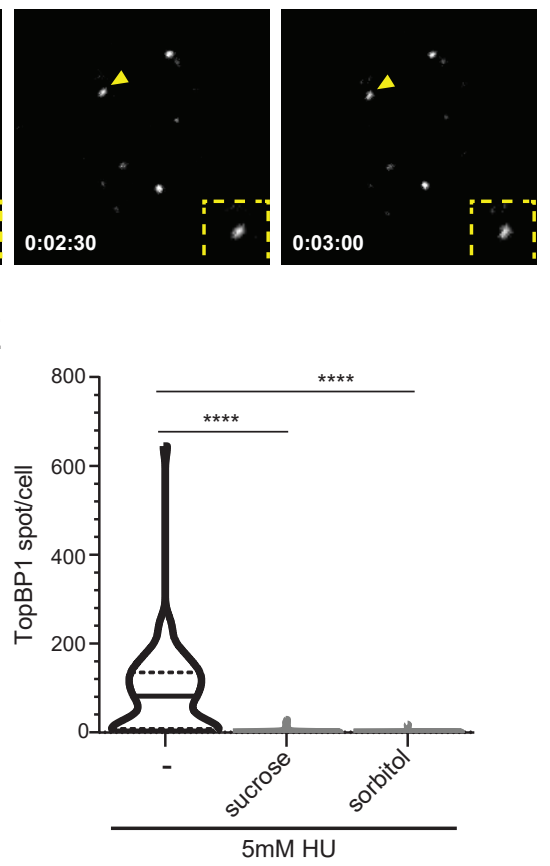

G

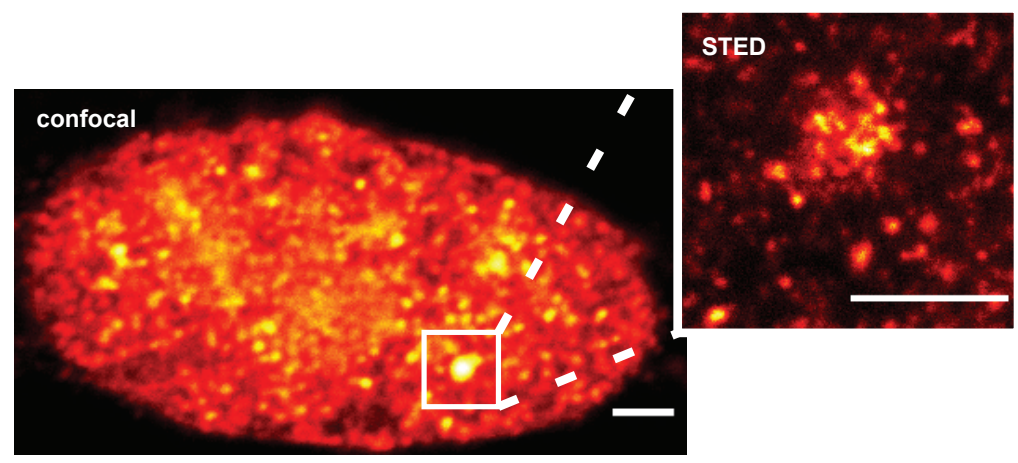

Figure 2 


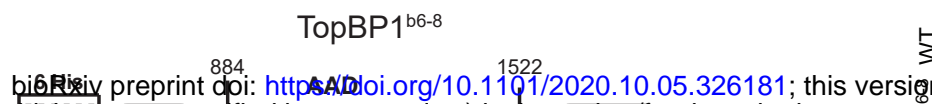

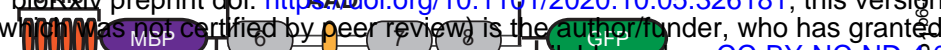
3C W1145

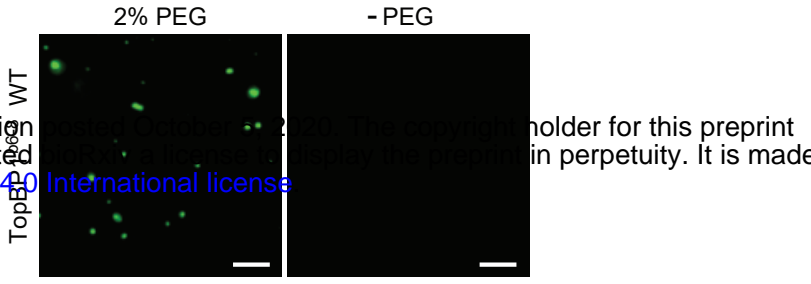

C
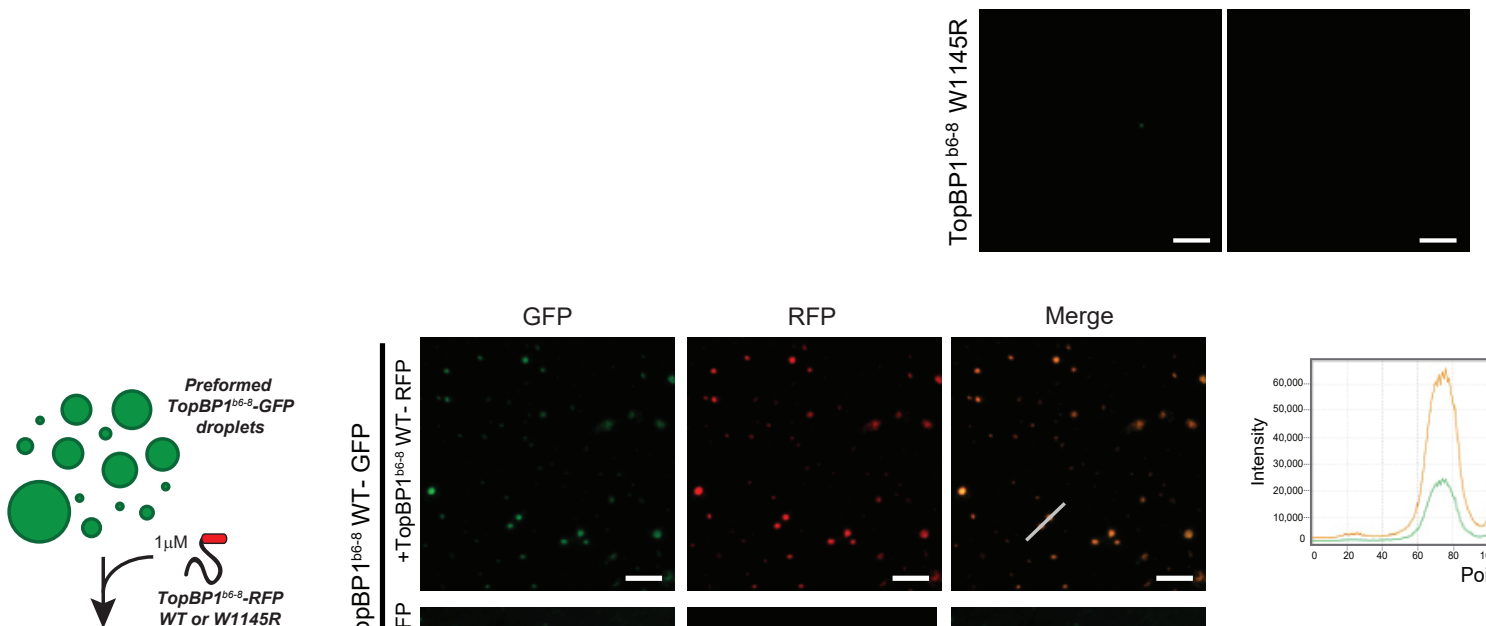

$\therefore \circ .0$
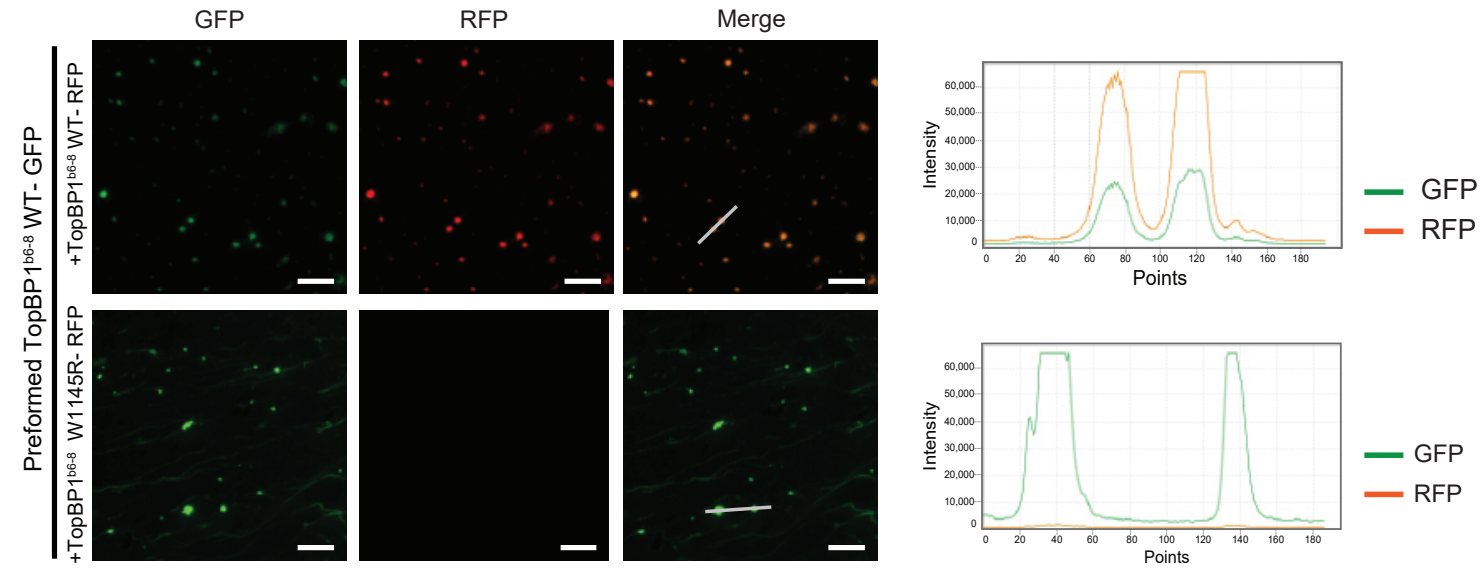

D
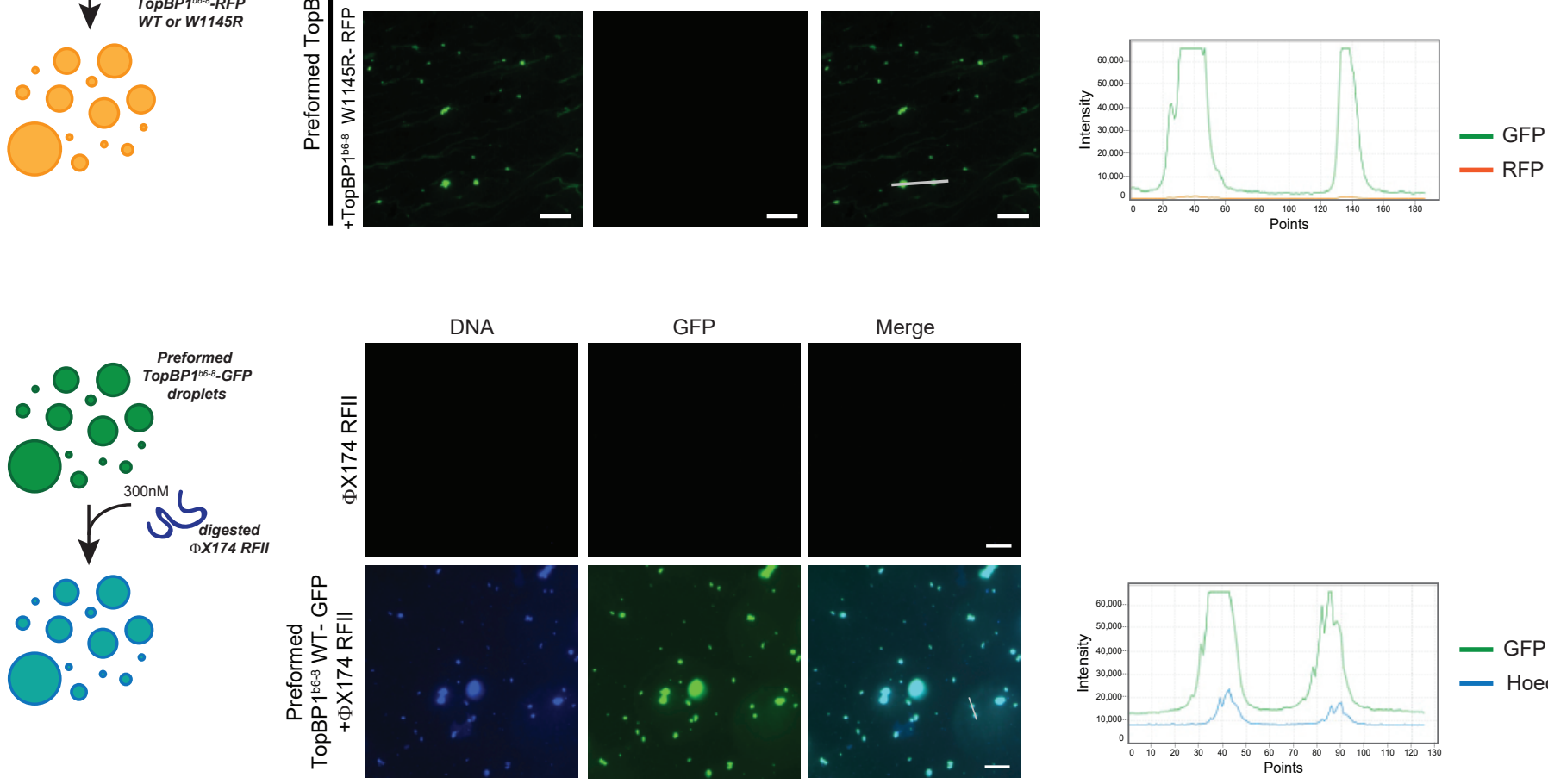

E

F

TopBP1 166-8 $\mathrm{WT}+2.9 \mathrm{~kb}$ plasmid DNA

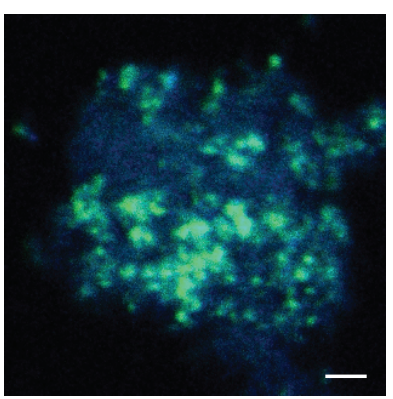

Nuclear Extract

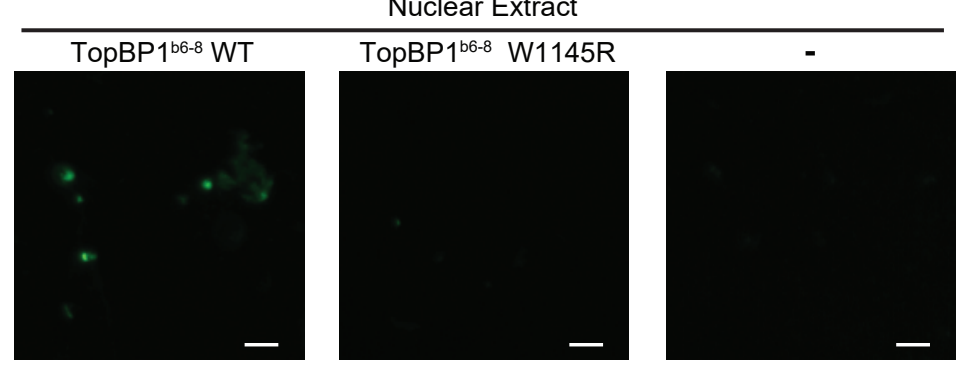

TopBP1 ${ }^{\mathrm{b6}-8}$ WT

$(10 \mu \mathrm{M})$ 
A

$$
\begin{gathered}
\text { Proximal } \\
\text { biotinylation }
\end{gathered}
$$

- Biotin

Proximal

bioRxiv preprint doi: https://doi.org/10.1101/2020.10.05.326181; this version Ddisteat October 5, 2020. The copyright holder for this preprint (which was not certified by peen review) is the author/funder, who has granted biditixir a license to display the preprint in perpetuity. It is made

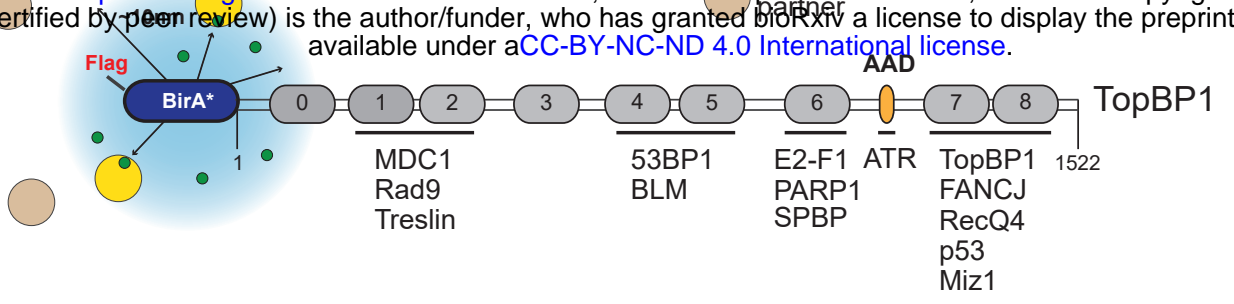

B
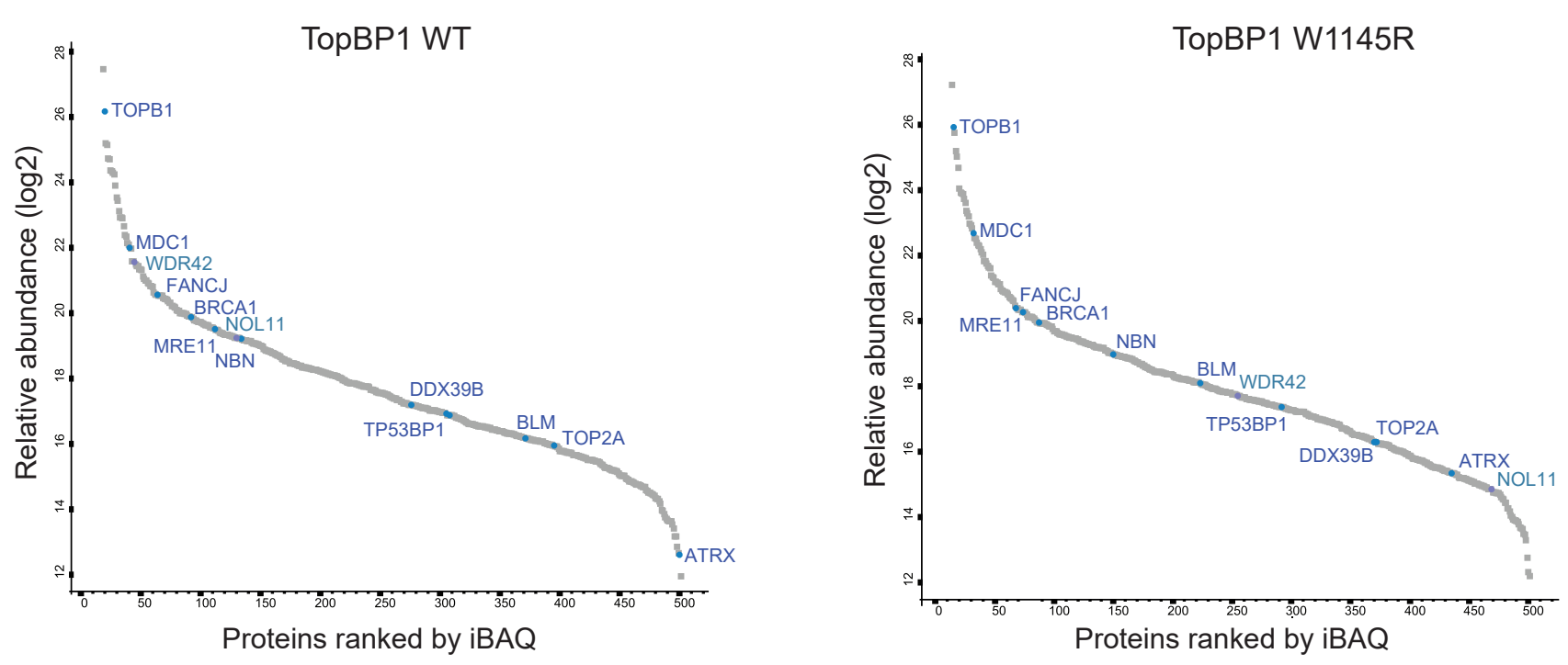

C

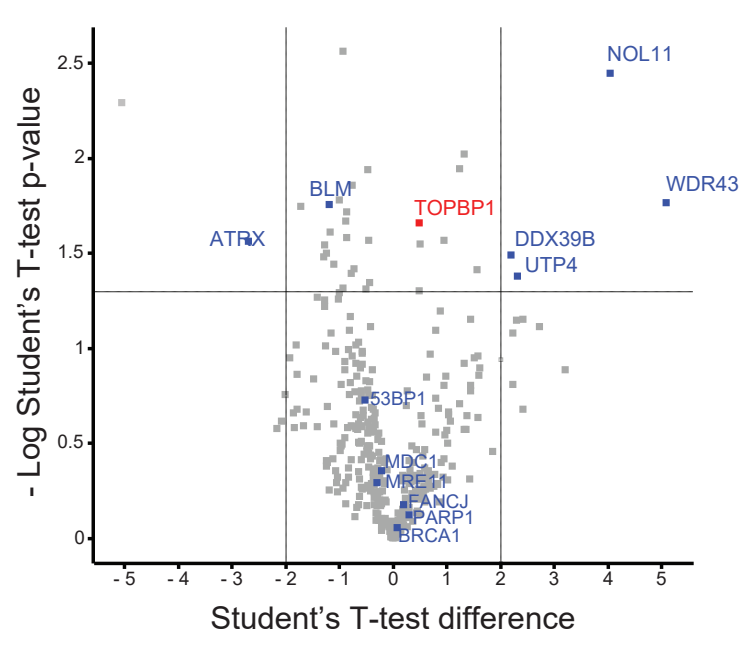

D

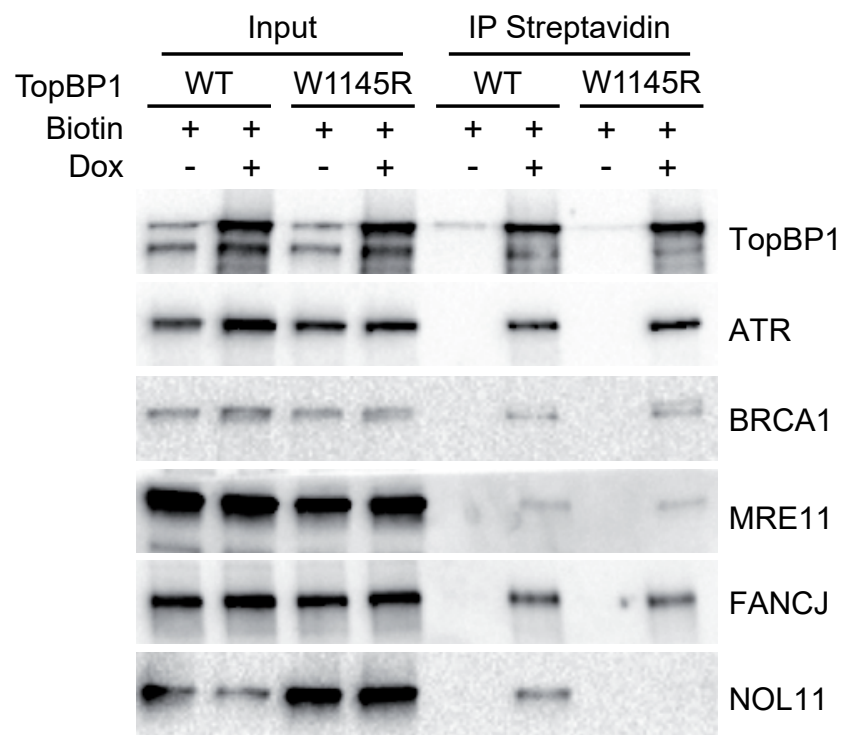


A

$$
\frac{\text { Light OFF }}{\frac{\alpha}{5}} \stackrel{\text { Light ON }}{\frac{\alpha}{5}}
$$

B

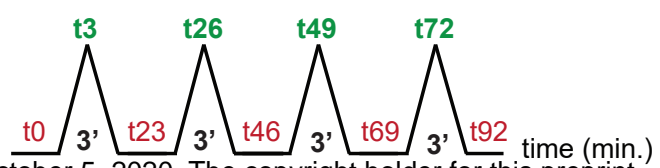

bioRxiv (which waפnot certified py pe review) is the author/funder, who has granted bioRxiva license to displaty the preprint in perpetuity. It is made
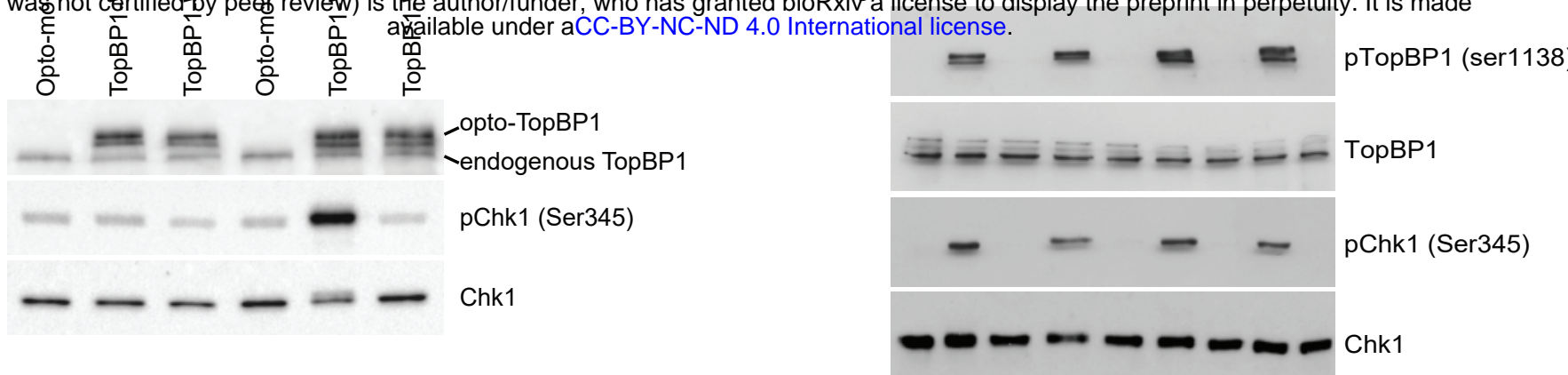

C

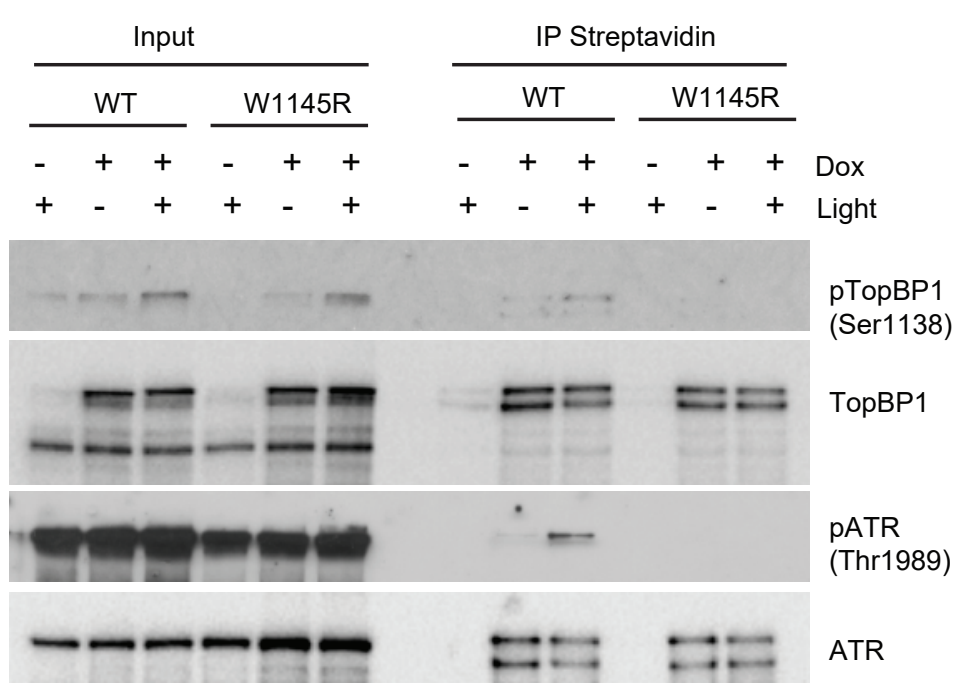

D
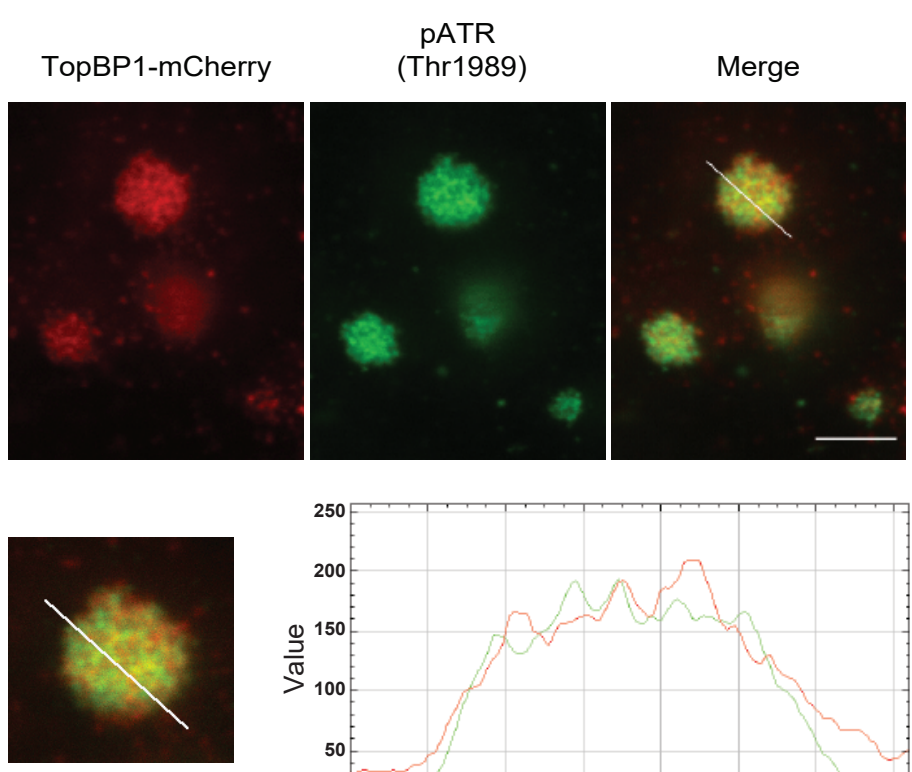

- pATR

— TopBP1

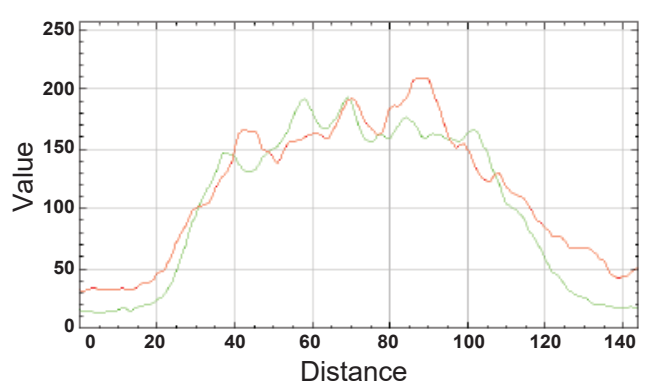

E

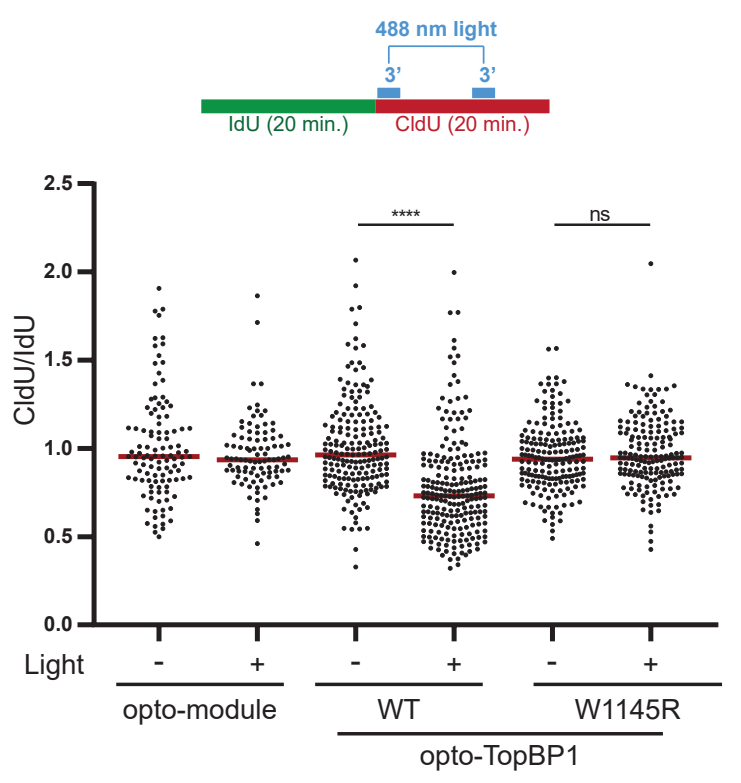


A

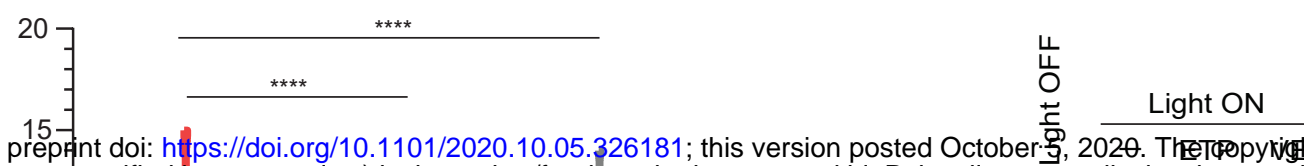

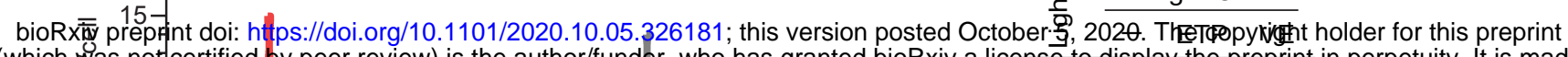
(which not certified by peer review) is the author/funder, who has granted bioRxiv a license to display the preprint in perpetuity. It is made

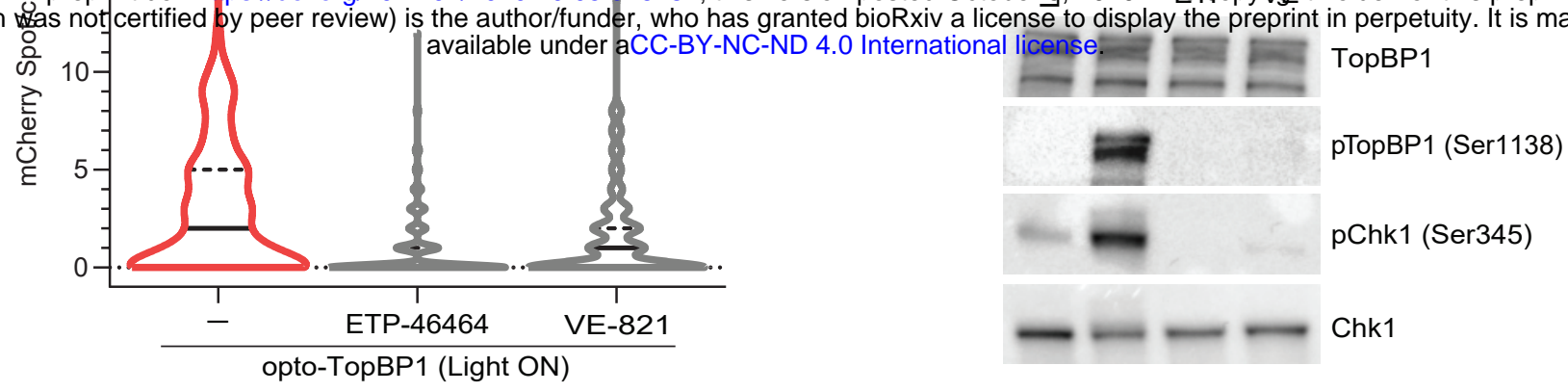

B

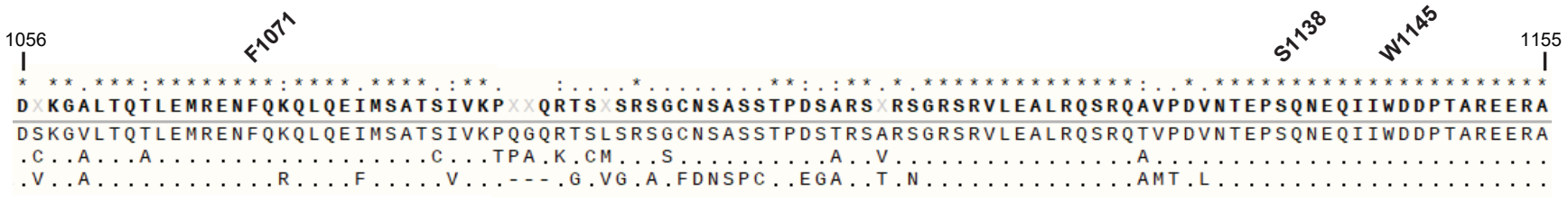

C
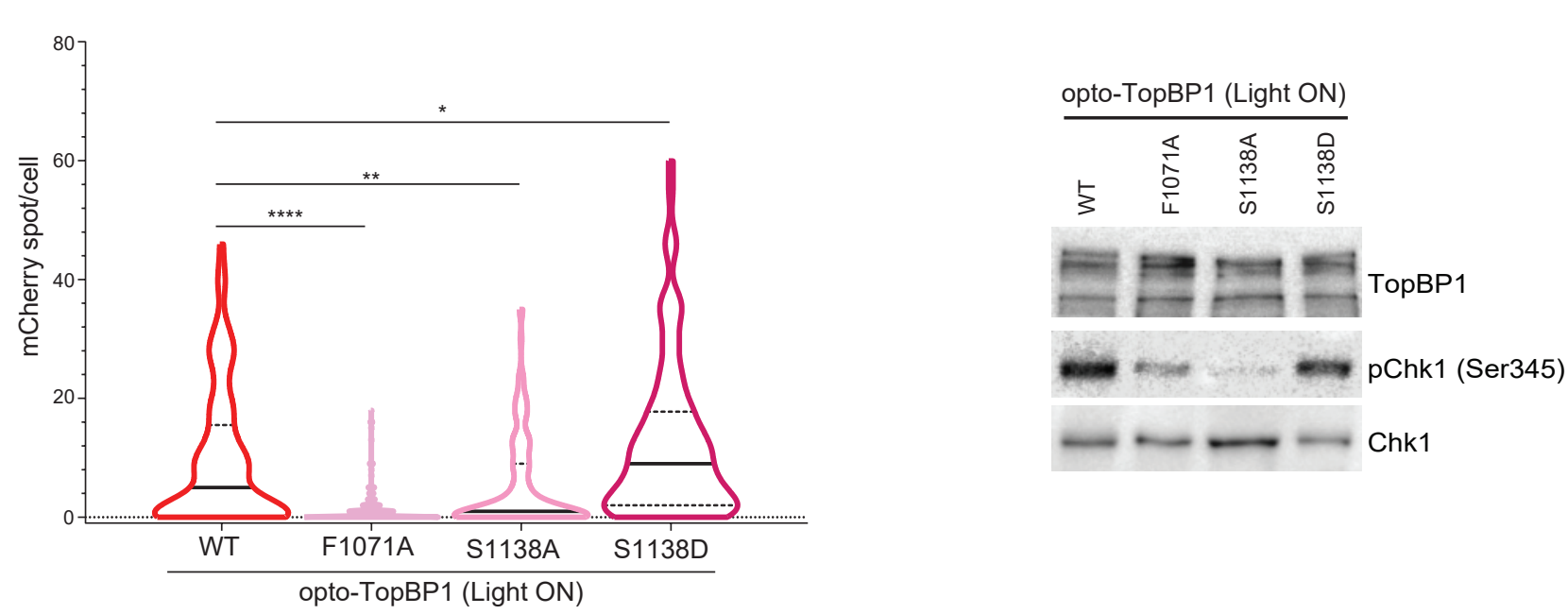

hTOpBP1 DSKGVLTQTLEMRENFQKQLQEIMSATSIVKPQGQRTSLSRSGCNSASSTPDSTRSARSGRSRVLEALRQSRQTVPDVNTEPSQNEQI IWDDPTAREERA

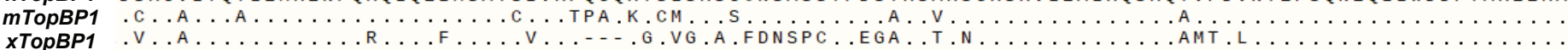

D

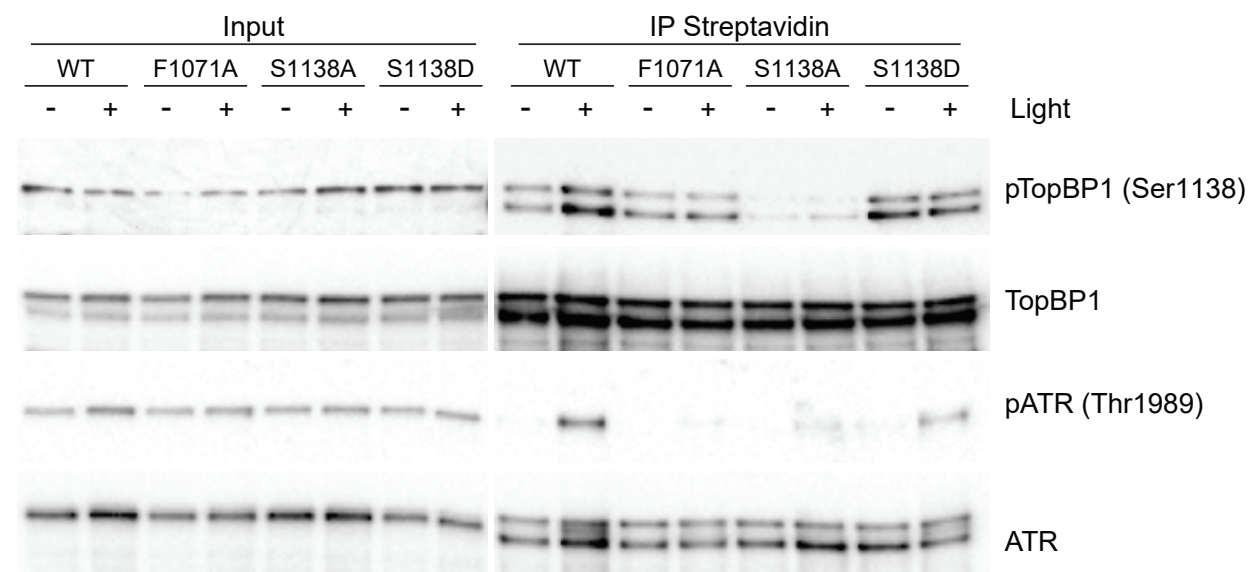

Figure 6 


\section{TopBP1 nucleation}

DNA replication impediments

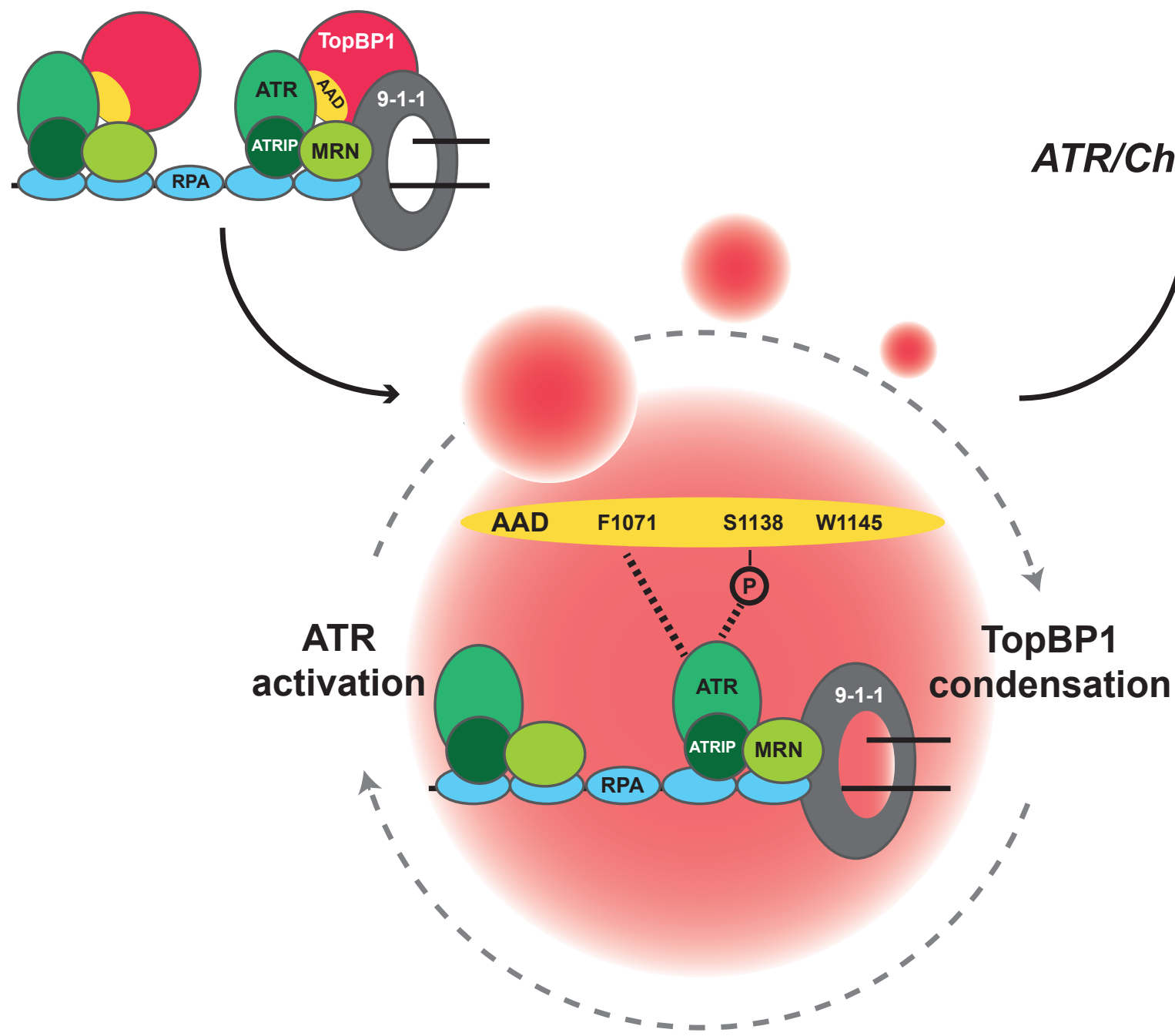

Signal amplification 
A

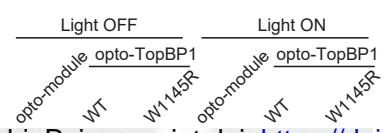

bioRxiv preprint doi: https://doi.org/10.1101/2020.10.05.326181; this version posted October 5, 2020. The copyright holder for this preprint (which was rotcertified by peer|review) is the author/funder, who has granted bioRxiv a license to display the preprint in perpetuity. It is made mcherry available under aCC-BY-NC-ND 4.0 International license.

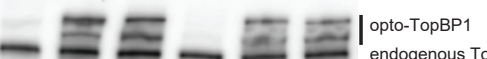

$-\infty-\omega_{\text {Tubulin }}$

B

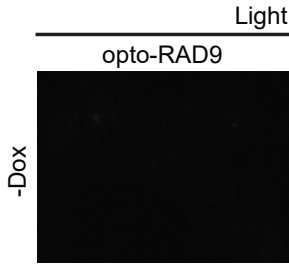

Light OFF
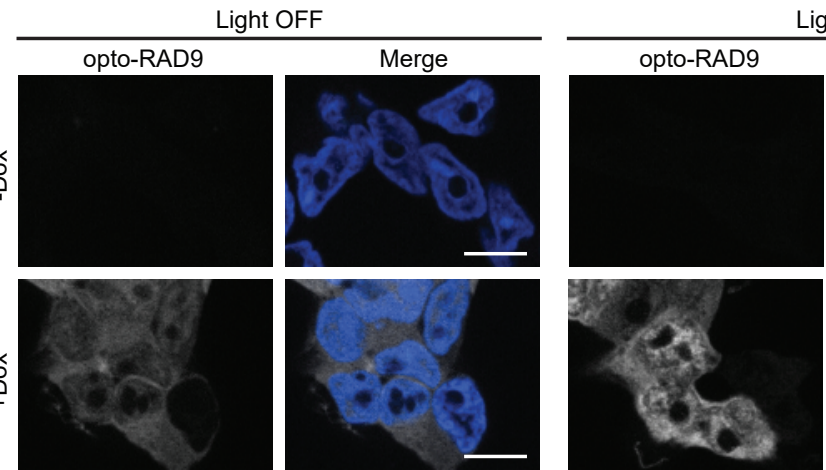

Light ON
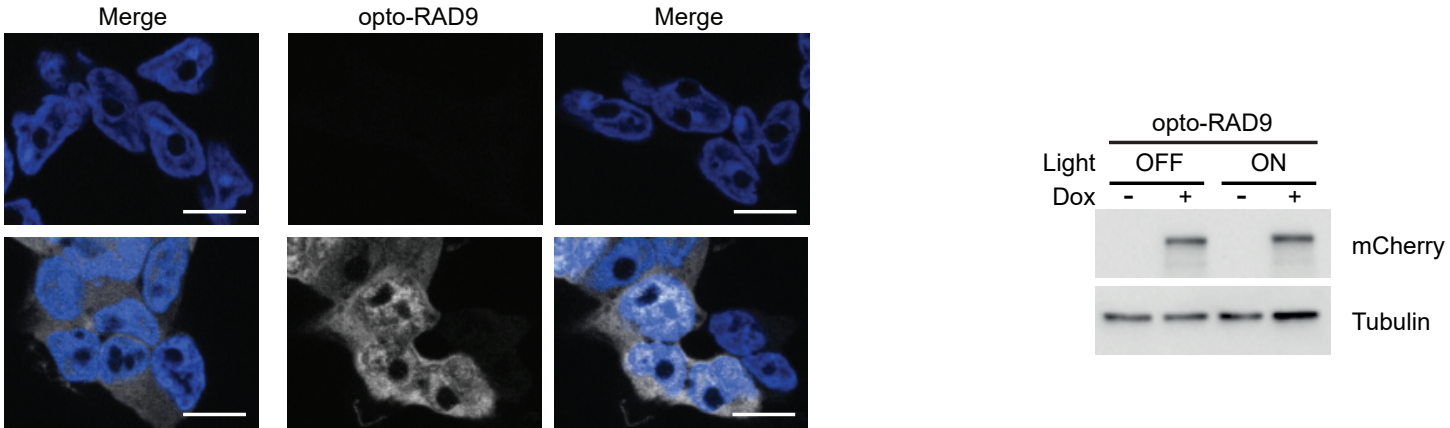

C
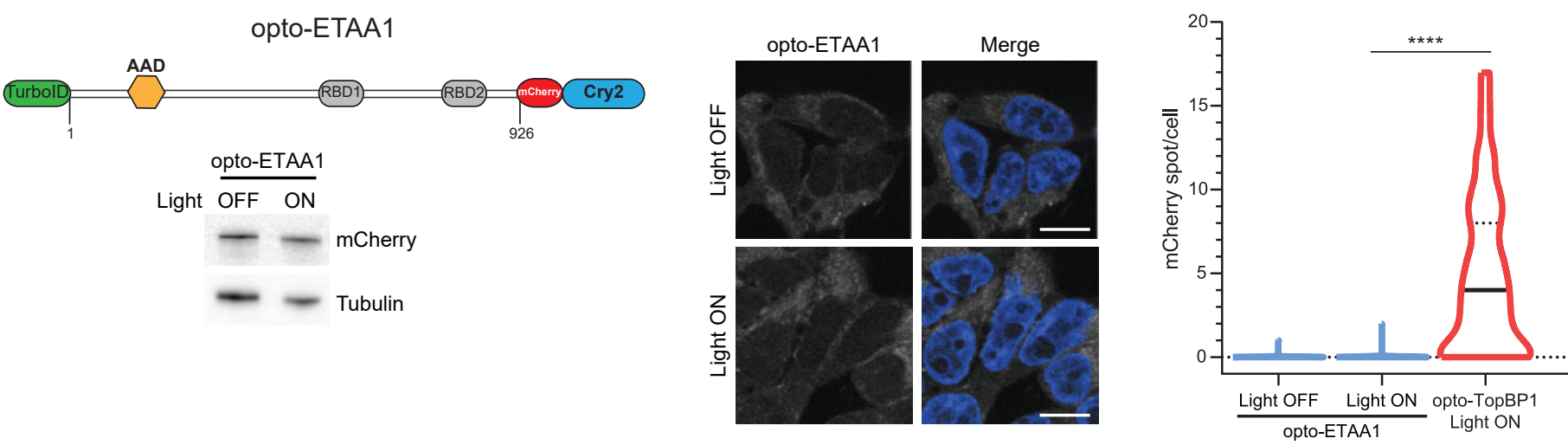
A

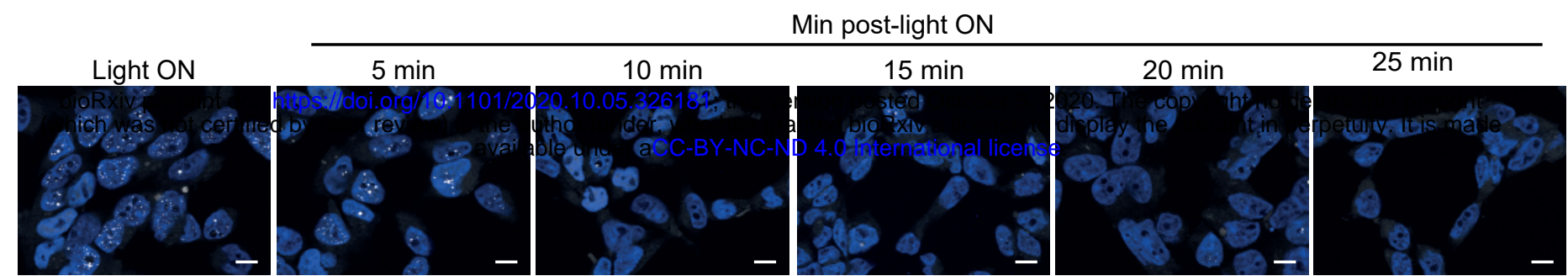

B
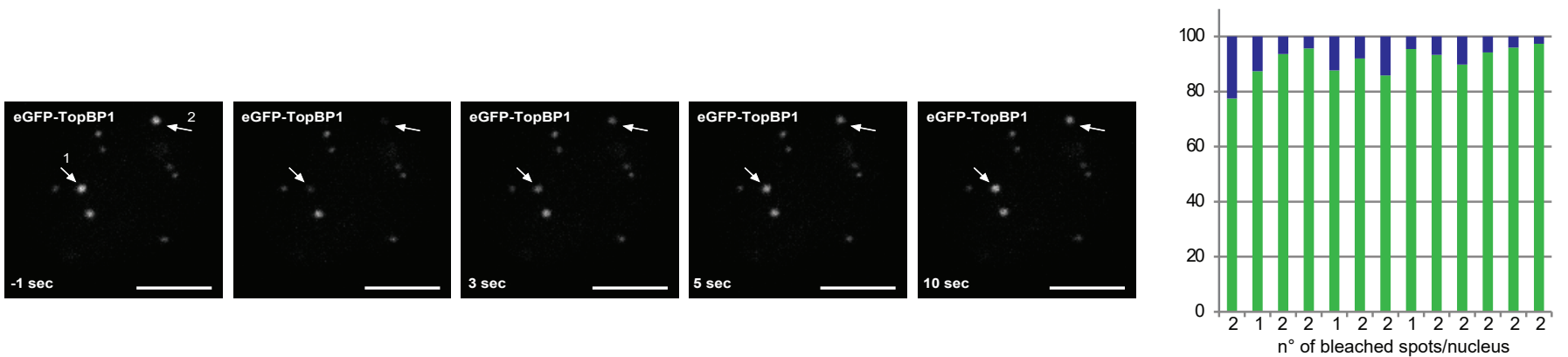

mobile fraction (\%) Mean $91,2 \%+/-5,5$

- immobile fraction (\%) Mean $8,8 \%+/-5,5$

C
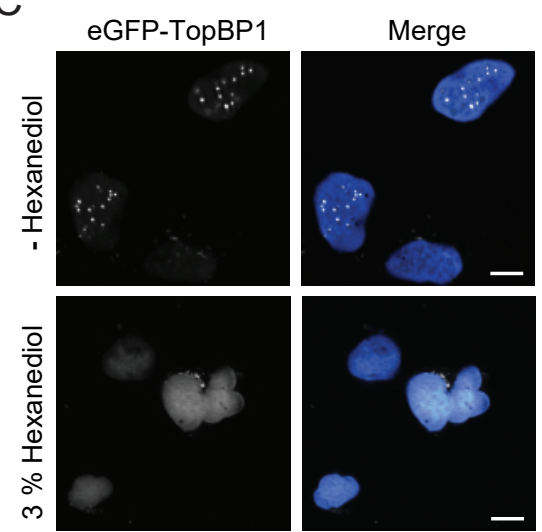

D
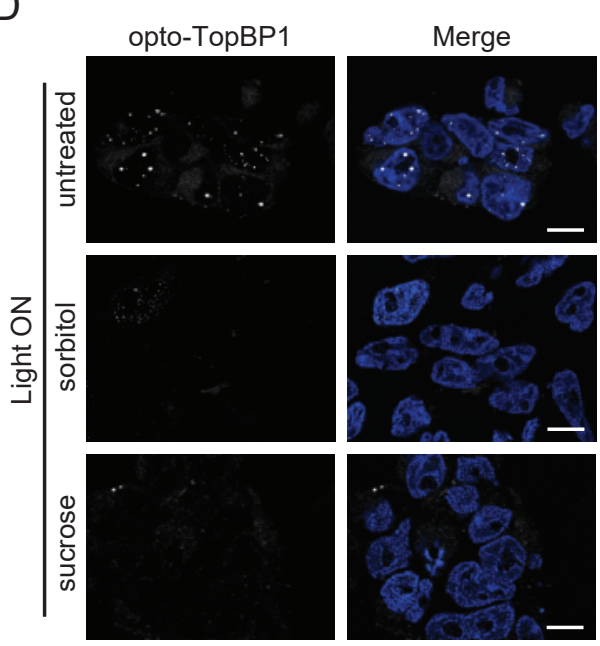

E

TopBP1
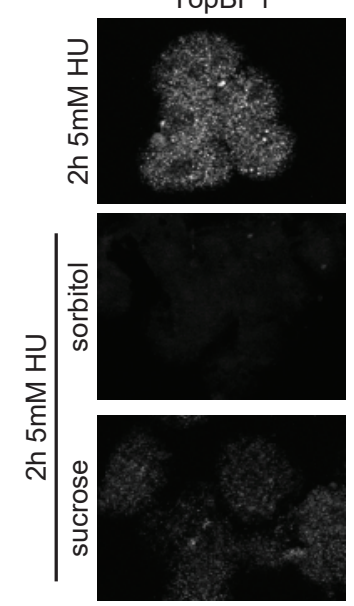

Merge
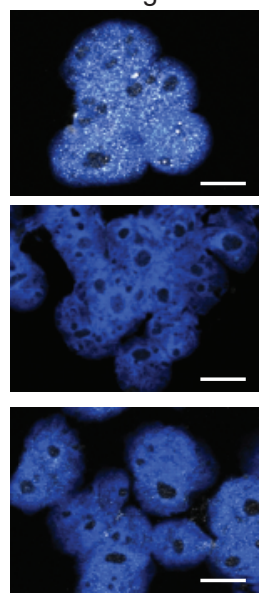

$\mathrm{F}$

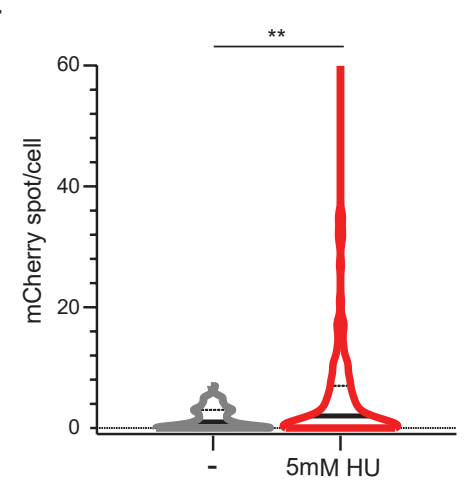


A
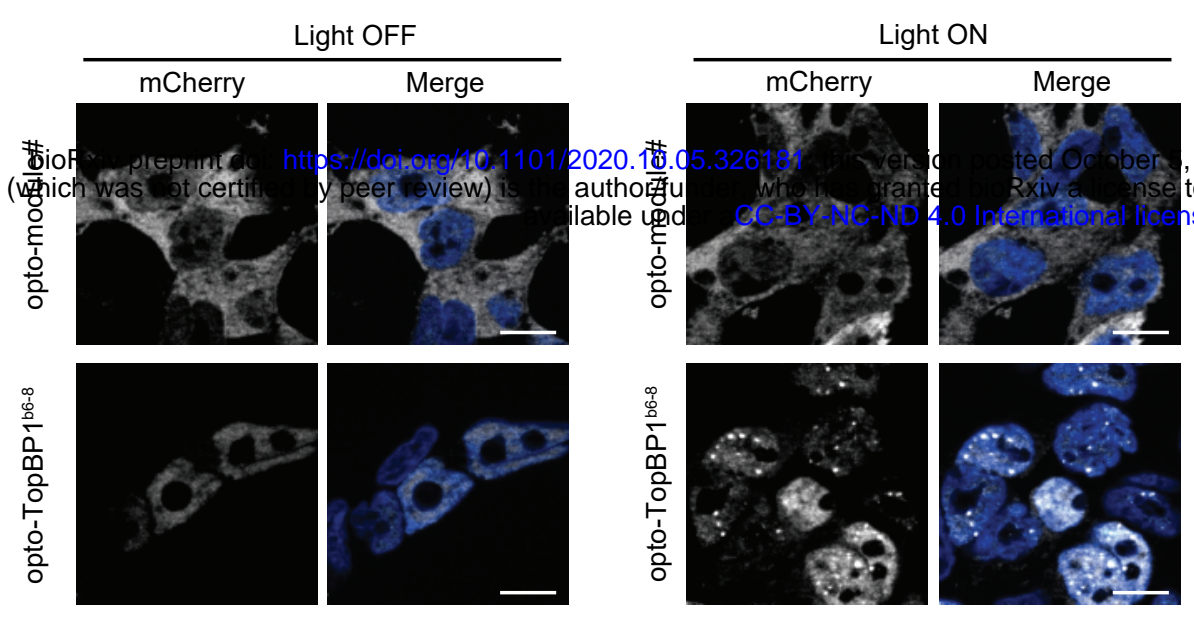

Light OFF Light ON

2020. The copyright holder for this preprint to display the preprint in pe⿶ppetuity. It is made

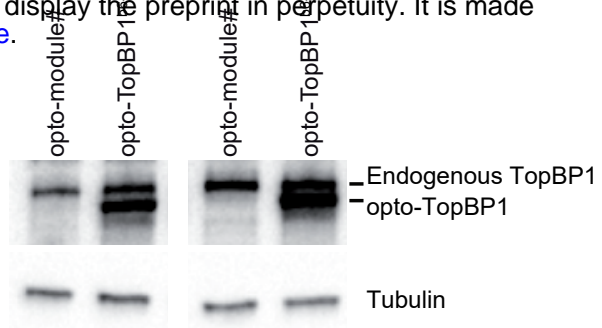

B

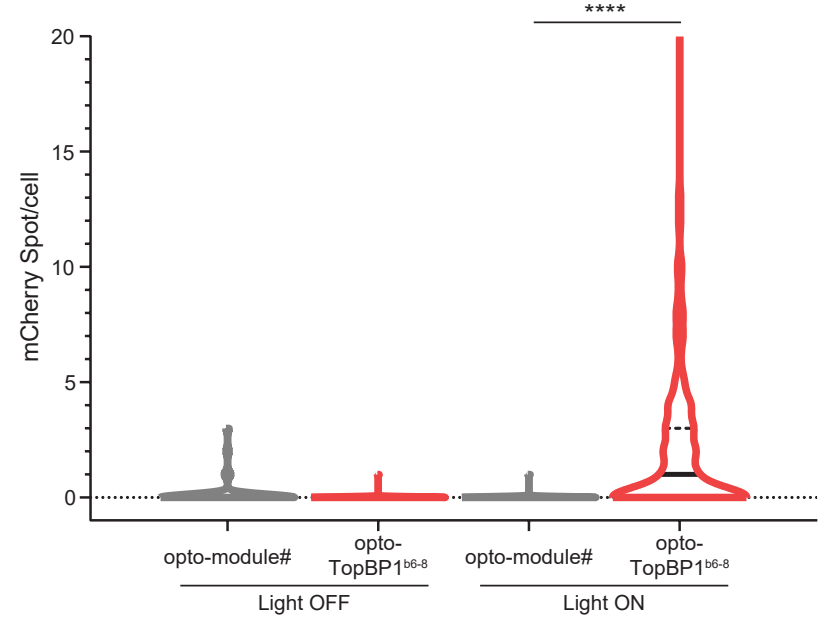
2\% PEG

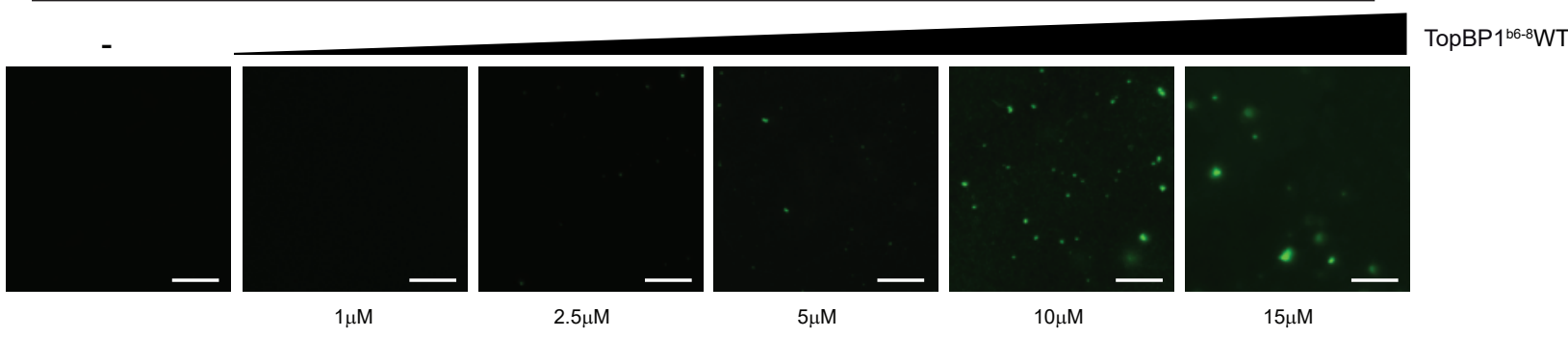

C

GFP

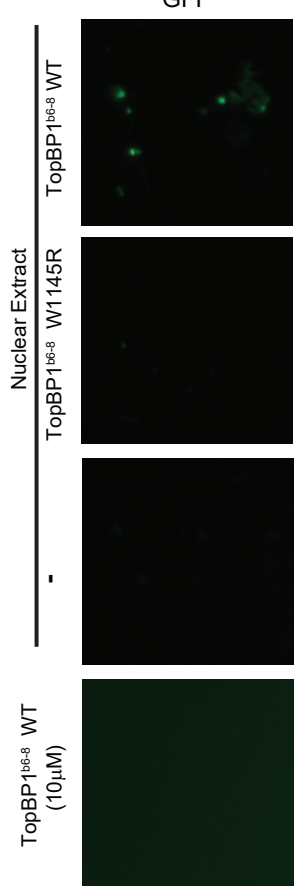

Merge
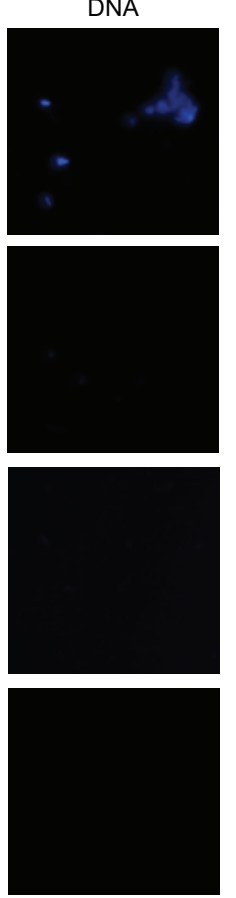
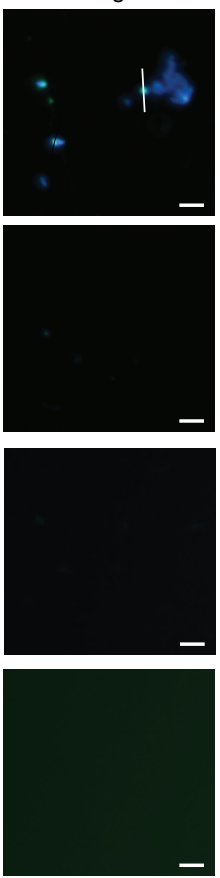

- GFP

- Hoechst

Suppl Figure 3_related to Figure 3 


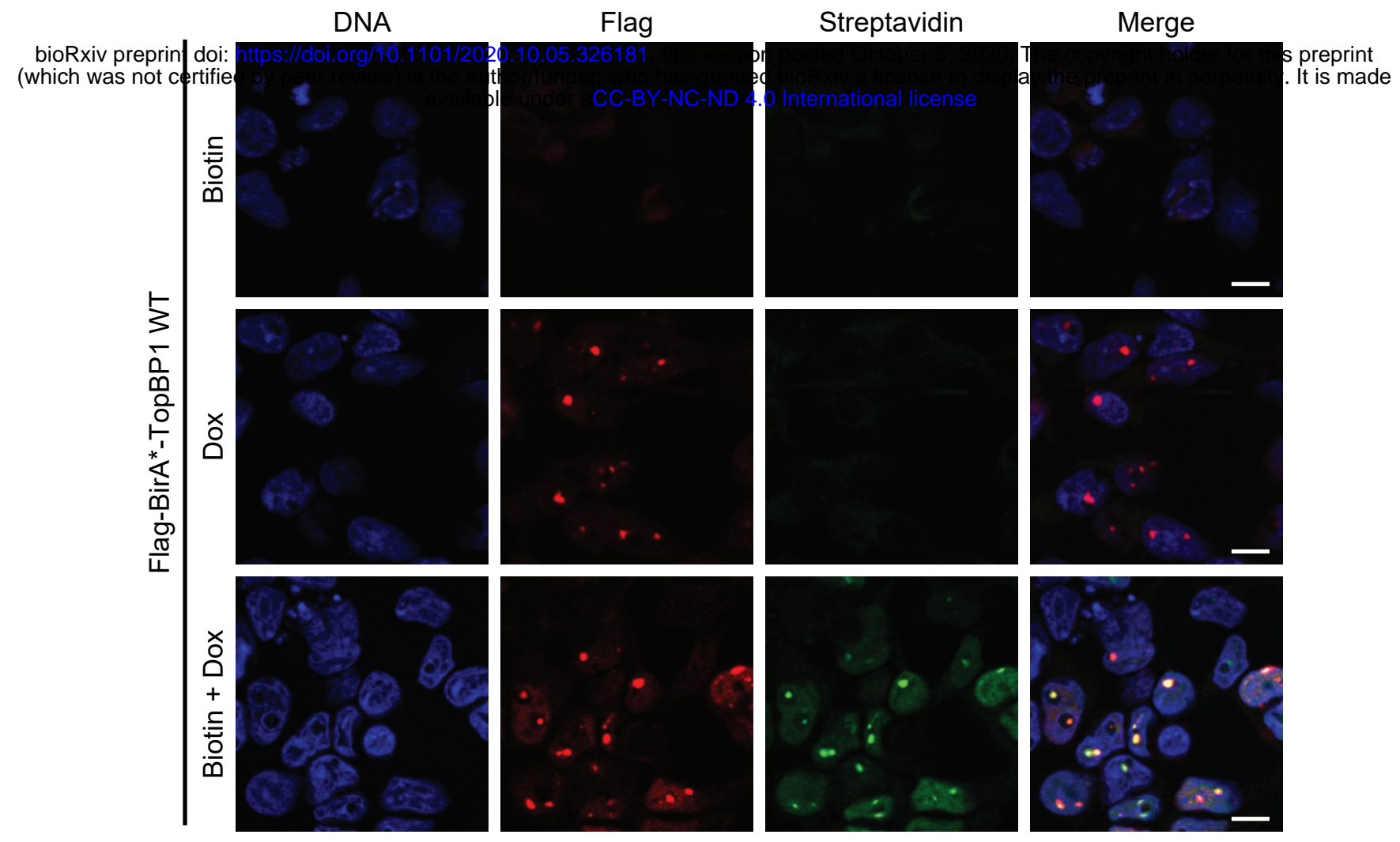


A

+ ETP46464

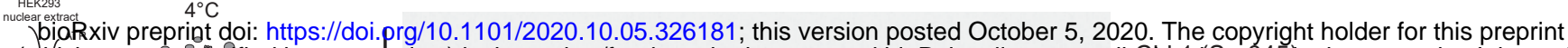

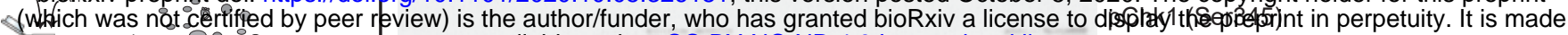
$1 . . .=4$ available under aCC-BY-NC-ND 4.0 International license.

$\because \cdots+\ldots$

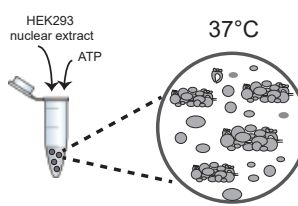

$37^{\circ} \mathrm{C}$

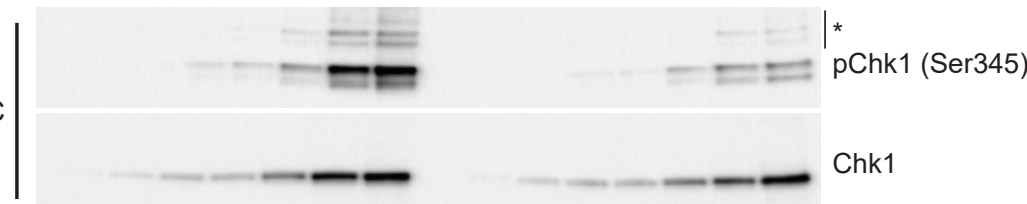

B

C
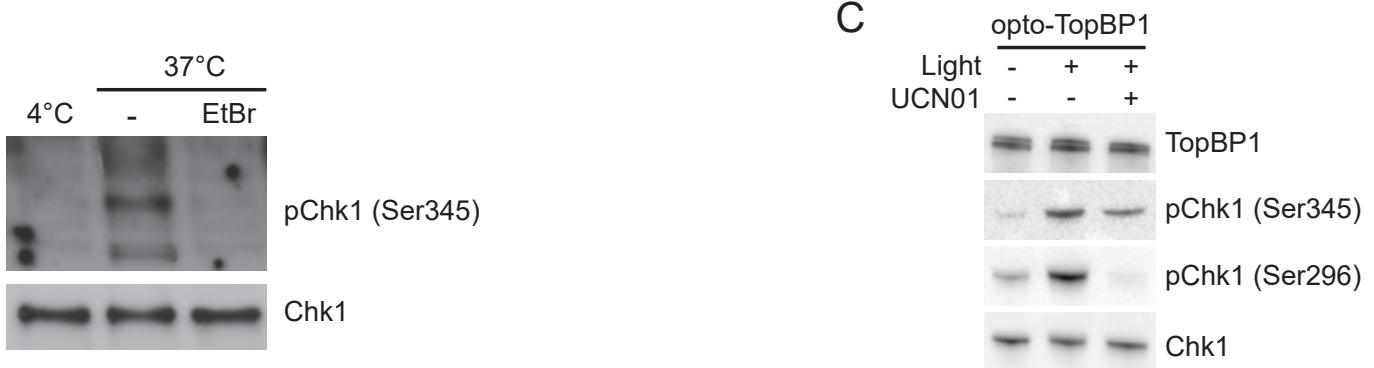

$\mathrm{D}$
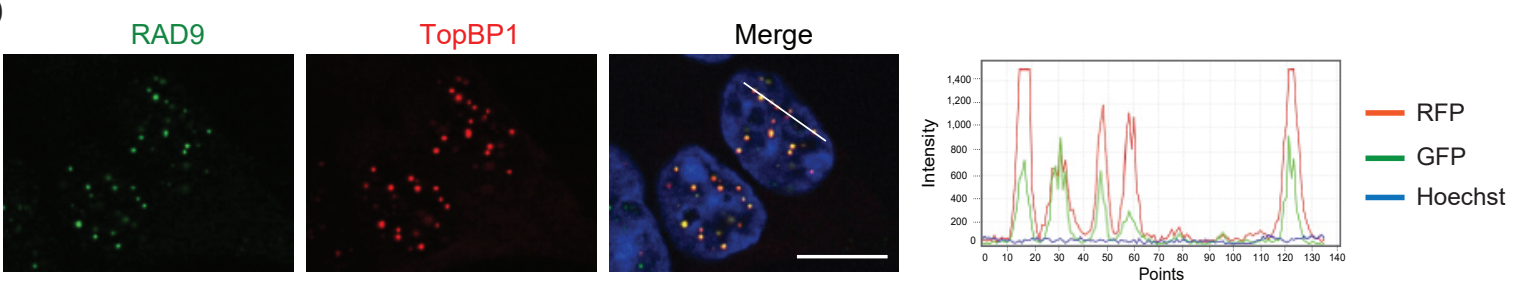

E

$\operatorname{pATR}(T 1989)$
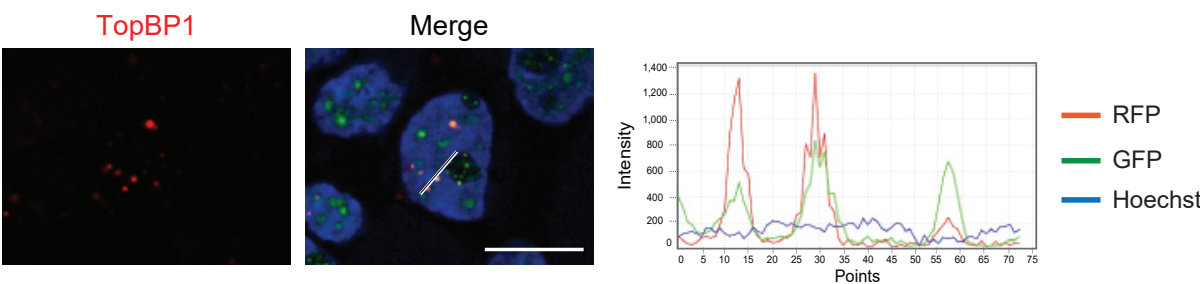

F

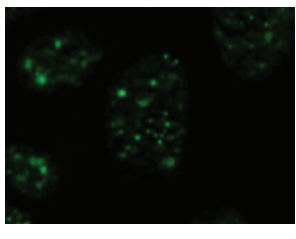

TopBP1

Merge
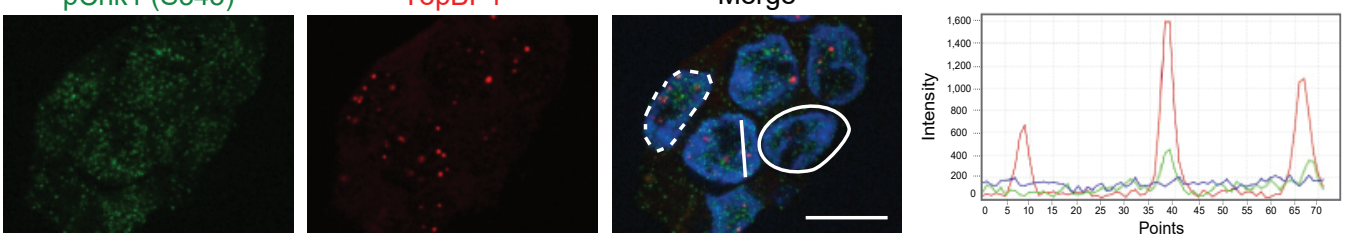

- RFP

- GFP

- Hoechst

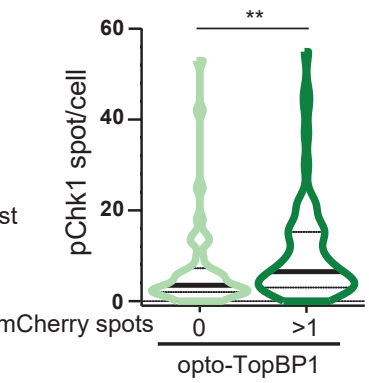

G

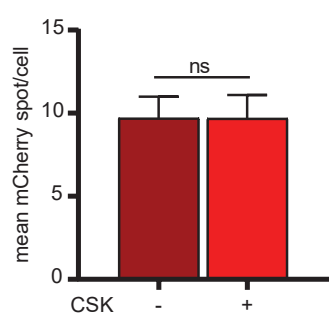


A

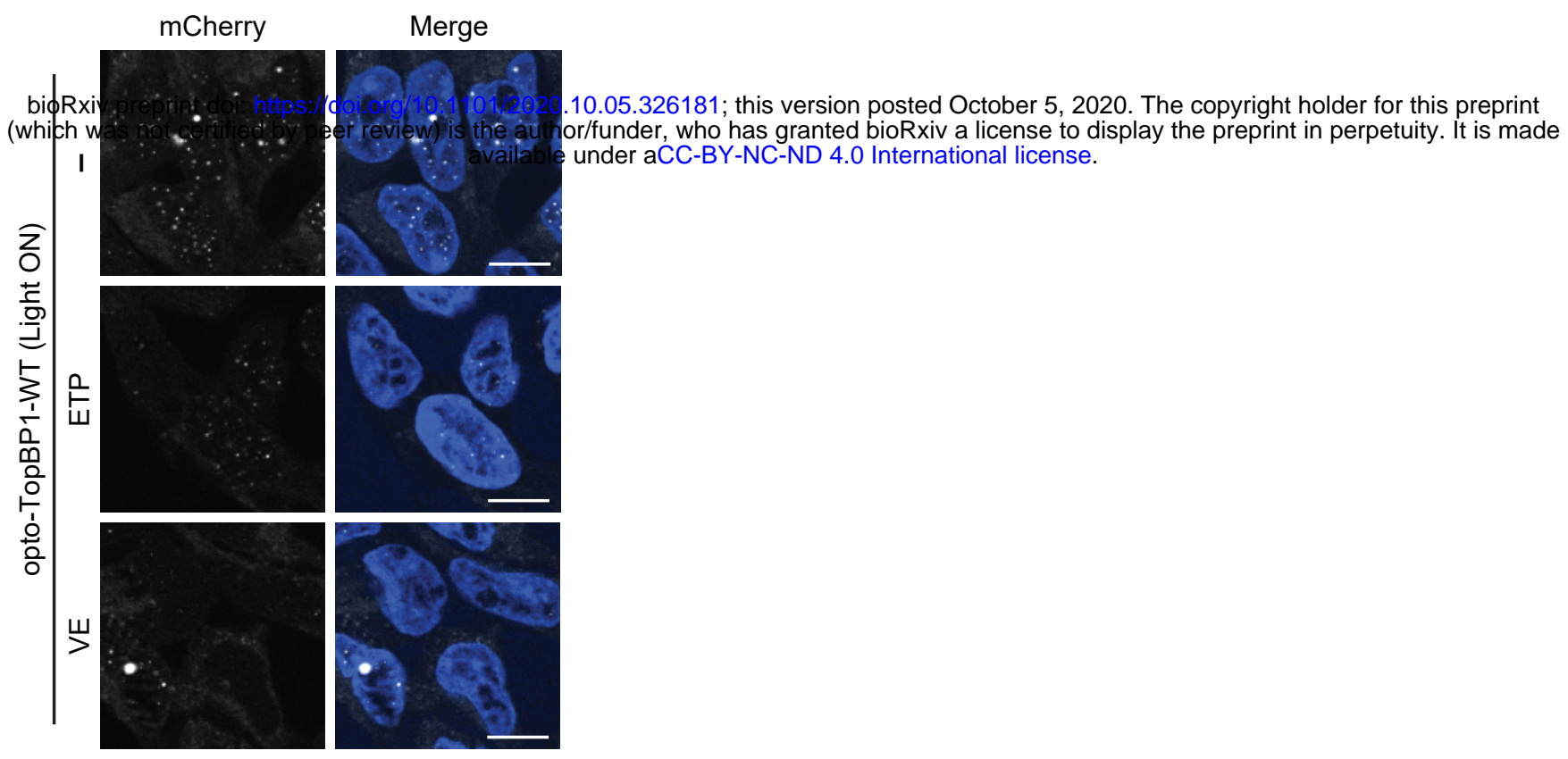

B

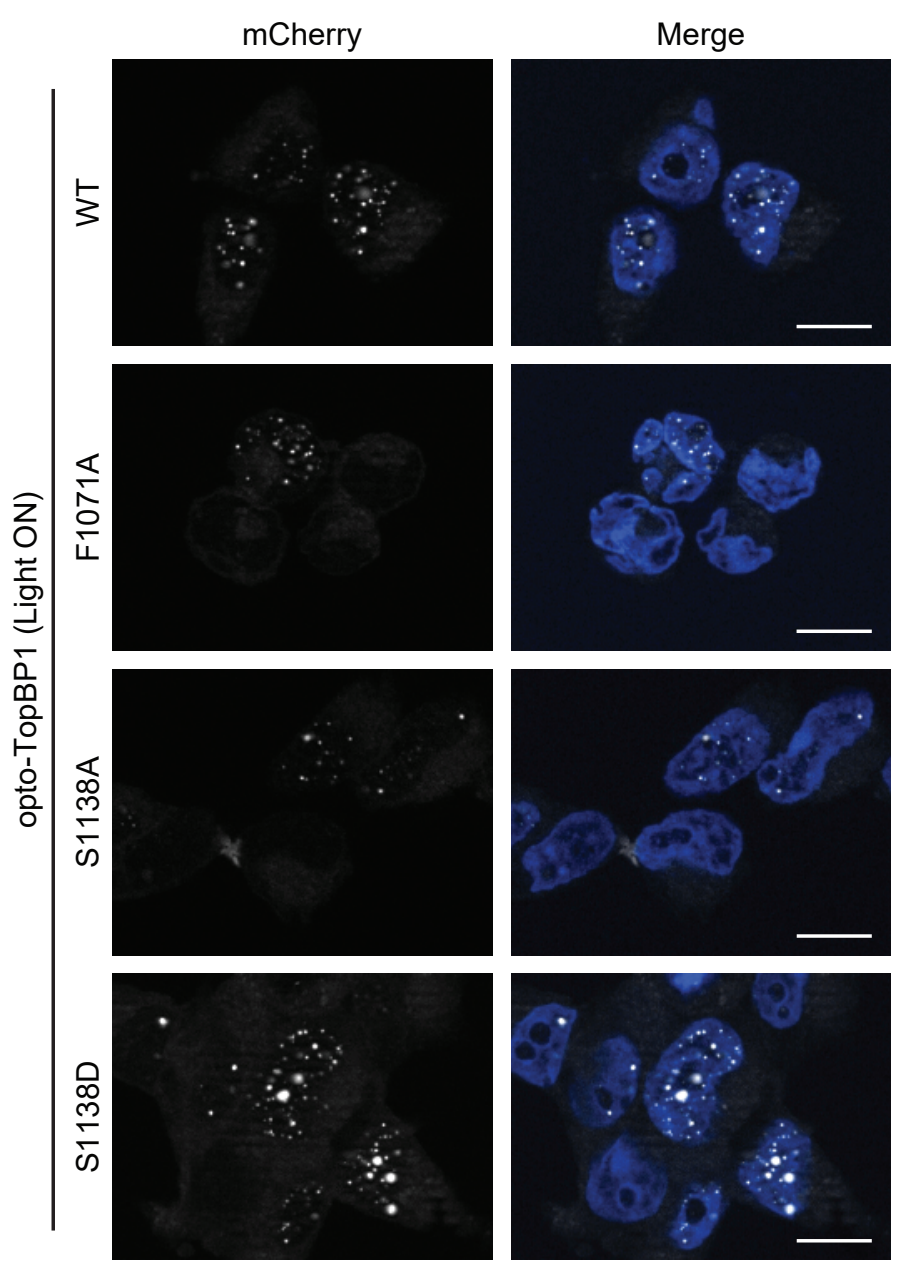

